\title{
Presburger Vector Addition Systems
}

\author{
Jérôme Leroux \\ LaBRI, UMR CNRS 5800, University of Bordeaux, Talence, France
}

\begin{abstract}
The reachability problem for Vector Addition Systems (VAS) is a central problem of net theory. The problem is known to be decidable by inductive invariants definable in the Presburger arithmetic. When the reachability set is definable in the Presburger arithmetic, the existence of such an inductive invariant is immediate. However, in this case, the computation of a Presburger formula denoting the reachability set is an open problem. In this paper we close this problem by proving that if the reachability set of a VAS is definable in the Presburger arithmetic, then the VAS is flatable, i.e. its reachability set can be obtained by runs labeled by words in a bounded language. As a direct consequence, classical algorithms based on acceleration techniques effectively compute a formula in the Presburger arithmetic denoting the reachability set.
\end{abstract}

Keywords-Infinite State Systems, Acceleration, flatability, Presburger, Vector Addition Systems, Petri nets, Reachability

\section{INTRODUCTION}

Vector Addition Systems (VAS) or equivalently Petri Nets are one of the most popular formal methods for the representation and the analysis of parallel processes [1]. The reachability problem is central since many computational problems (even outside the realm of parallel processes) reduce to this problem. Sacerdote and Tenney provided in [2] a partial proof of decidability of this problem. The proof was completed in 1981 by Mayr [3] and simplified by Kosaraju [4] from [2], [3]. Ten years later [5], Lambert provided a further simplified version based on [4]. This last proof still remains difficult and the upper-bound complexity of the corresponding algorithm is just known to be non-primitive recursive. Nowadays, the exact complexity of the reachability problem for VAS is still an open-question. Even an Ackermannian upper bound is open (this bound holds for VAS with finite reachability sets [6]).

Recently, in [7], the reachability sets of VAS are proved to be almost semilinear, a class of sets that extends the class of Presburger sets (the sets definable in $\mathrm{FO}(\mathbb{Z},+, \leq)$ ) inspired by the semilinear sets [8]. Note that in general reachability sets are not definable in the Presburger arithmetic [9]. An application of the almost semilinear sets was provided; a final configuration is not reachable from an initial one if and only if there exists a forward inductive invariant definable in the Presburger arithmetic that contains the initial configuration but not the final one. Since we can decide if a Presburger formula denotes a forward inductive invariant, we deduce that there exist checkable certificates of non-reachability in the Presburger arithmetic. In particular, there exists a simple algorithm for deciding the general VAS reachability problem based on two semi-algorithms. A first one that tries to prove the reachability by enumerating finite sequences of actions and a second one that tries to prove the non-reachability by enumerating Presburger formulas. Such an algorithm always terminates in theory but in practice an enumeration does not provide an efficient way for deciding the reachability problem. In particular the problem of deciding efficiently the reachability problem is still an open question.

When the reachability set is definable in the Presburger arithmetic, the existence of checkable certificates of nonreachability in the Presburger arithmetic is immediate since the reachability set is a forward inductive invariant (in fact the most precise one). The problem of deciding if the reachability set of a VAS is definable in the Presburger arithmetic was studied twenty years ago independently by Dirk Hauschildt during his $\mathrm{PhD}$ [10] and Jean-Luc Lambert. Unfortunately, these two works were never published. Moreover, from these works, it is difficult to deduce a simple algorithm for computing a Presburger formula denoting the reachability set when such a formula exists.

For the class of flatable vector addition systems [11], [12], such a computation can be performed with accelerations techniques. Let us recall that a VAS is said to be flatable if there exists a language included in $w_{1}^{*} \ldots w_{m}^{*}$ for some words $w_{1}, \ldots, w_{m}$ such that every reachable configuration is reachable by a run labeled by a word in this language (such a language is said to be bounded [13]). Acceleration techniques provide a framework for deciding reachability properties that works well in practice but without termination guaranty in theory. Intuitively, acceleration techniques consist in computing with some symbolic representations transitive closures of sequences of actions. For vector addition systems, the Presburger arithmetic is known to be expressive enough for this computation. As a direct consequence, when the reachability set of a vector addition system is computable with acceleration techniques, this set is necessarily definable in the Presburger arithmetic. In [12], we proved that a VAS is flatable if, and only if, its reachability set is computable by acceleration.

Recently, we proved that many classes of VAS with known Presburger reachability sets are flatable [12] and we conjectured that VAS with reachability sets definable in the Presburger arithmetic are flatable. In this paper, we prove this conjecture. As a direct consequence, classical acceleration techniques always terminate on the computation of Presburger formulas denoting reachability sets of VAS when such a formula exists.

Outline In section III we introduce the acceleration framework and the notion of flatable subreachability sets and flat- 
able subreachability relations. We also recall why Presburger formulas denoting reachability sets of flatable VAS are computable with acceleration techniques. In section IV we recall the definition of well-preorders, the Dickson's lemma and the Higman's lemma. In Section V we provide some classical elements of linear algebra. We recall the characterization of Presburger sets as finite union of linear sets. We also introduce in this section the central notion of smooth periodic sets. Intuitively smooth periodic sets are sets of vectors of rational numbers stable by finite sums, and such that from any infinite sequence of elements, a so-called limit vector can be extracted. The definition of smooth periodic sets also requires that the possible limits forms a set definable in the first order logic FO $(\mathbb{Q},+, \leq)$. In Section VI we recall the well-order over the runs first introduced in [14] central in the analysis of vector addition systems. Sections VII and VIII provide independent results that are used in Section IX to prove that reachability sets of vector additions systems intersected with Presburger sets are finite unions of sets $\mathbf{b}+\mathbf{P}$ where $\mathbf{b}$ is a vector and $\mathbf{P}$ is a smooth periodic set such that for every linear set $\mathbf{Y} \subseteq \mathbf{b}+\mathbf{P}$ there exists $\mathbf{p} \in \mathbf{P}$ such that $\mathbf{p}+\mathbf{Y}$ is a flatable subreachability set (intuitively a subset of the reachability set computable by acceleration). The last Sections X and XI show that this decomposition of the reachability set is sufficient for proving that if the reachability set of a VAS is definable in the Presburger arithmetic then it is flatable.

\section{Vectors AND Numbers}

We denote by $\mathbb{N}, \mathbb{N}_{>0}, \mathbb{Z}, \mathbb{Q}, \mathbb{Q}_{\geq 0}, \mathbb{Q}_{>0}$ the set of natural numbers, positive integers, integers, rational numbers, non negative rational numbers, and positive rational numbers. Vectors and sets of vectors are denoted in bold face. The $i$ th component of a vector $\mathbf{v} \in \mathbb{Q}^{d}$ is denoted by $\mathbf{v}(i)$. We introduce $\|\mathbf{v}\|_{\infty}=\max _{1<i<d}|\mathbf{v}(i)|$ where $|\mathbf{v}(i)|$ is the absolute value of $\mathbf{v}(i)$. A set $\mathbf{B} \subseteq \mathbb{Q}^{d}$ is said to be bounded if there exists $m \in \mathbb{Q}_{\geq 0}$ such that $\|\mathbf{b}\|_{\infty} \leq m$ for every $\mathbf{b} \in \mathbf{B}$. The addition function + is extended component-wise over $\mathbb{Q}^{d}$.

The dot product of two vectors $\mathbf{x}, \mathbf{y} \in \mathbb{Q}^{d}$ is the rational number $\sum_{i=1}^{d} \mathbf{x}(i) \mathbf{y}(i)$ denoted by $\mathbf{x} \cdot \mathbf{y}$.

Given two sets $\mathbf{V}_{1}, \mathbf{V}_{2} \subseteq \mathbb{Q}^{d}$ we denote by $\mathbf{V}_{1}+\mathbf{V}_{2}$ the set $\left\{\mathbf{v}_{1}+\mathbf{v}_{2} \mid\left(\mathbf{v}_{1}, \mathbf{v}_{2}\right) \in \mathbf{V}_{1} \times \mathbf{V}_{2}\right\}$, and we denote by $\mathbf{V}_{1}-\mathbf{V}_{2}$ the set $\left\{\mathbf{v}_{1}-\mathbf{v}_{2} \mid\left(\mathbf{v}_{1}, \mathbf{v}_{2}\right) \in \mathbf{V}_{1} \times \mathbf{V}_{2}\right\}$. In the same way given $T \subseteq \mathbb{Q}$ and $\mathbf{V} \subseteq \mathbb{Q}^{d}$ we let $T \mathbf{V}=\{t \mathbf{v} \mid(t, \mathbf{v}) \in T \times \mathbf{V}\}$ We also denote by $\mathbf{v}_{1}+\mathbf{V}_{2}$ and $\mathbf{V}_{1}+\mathbf{v}_{2}$ the sets $\left\{\mathbf{v}_{1}\right\}+\mathbf{V}_{2}$ and $\mathbf{V}_{1}+\left\{\mathbf{v}_{2}\right\}$, and we denote by $t \mathbf{V}$ and $T \mathbf{v}$ the sets $\{t\} \mathbf{V}$ and $T\{\mathbf{v}\}$. In the sequel, an empty sum of sets included in $\mathbb{Q}^{d}$ denotes the set reduced to the zero vector $\{\mathbf{0}\}$.

\section{iII. Flatable Vector Addition Systems}

A Vector Addition System (VAS) is a pair $\left(\mathbf{c}_{\text {init }}, \mathbf{A}\right)$ where $\mathbf{c}_{\text {init }} \in \mathbb{N}^{d}$ is an initial configuration and $\mathbf{A} \subseteq \mathbb{Z}^{d}$ is a finite set of actions.

The semantics of vector addition systems is obtained as follows. A vector $\mathbf{c} \in \mathbb{N}^{d}$ is called a configuration. We introduce the labeled relation $\rightarrow$ defined by $\mathbf{x} \stackrel{\text { a }}{\rightarrow} \mathbf{y}$ if $\mathbf{x}, \mathbf{y} \in \mathbb{N}^{d}$ are configurations, $\mathbf{a} \in \mathbf{A}$ is an action, and $\mathbf{y}=\mathbf{x}+\mathbf{a}$. As expected, a run is a non-empty word $\rho=\mathbf{c}_{0} \ldots \mathbf{c}_{k}$ of configurations $\mathbf{c}_{j} \in \mathbb{N}^{d}$ such that $\mathbf{a}_{j}=\mathbf{c}_{j}-\mathbf{c}_{j-1}$ is a vector in A (see e.g., Figure 1). The word $w=\mathbf{a}_{1} \ldots \mathbf{a}_{k}$ is called the label of $\rho$. The configurations $\mathbf{c}_{0}$ and $\mathbf{c}_{k}$ are respectively called the source and the target and they are denoted by $\operatorname{src}(\rho)$ and $\operatorname{tgt}(\rho)$. We also denote by $\operatorname{dir}(\rho)$ the pair $(\operatorname{src}(\rho), \operatorname{tgt}(\rho))$ called the direction of $\rho$. The relation $\rightarrow$ is extended over the words $w=\mathbf{a}_{1} \ldots \mathbf{a}_{k}$ of actions $\mathbf{a}_{j} \in \mathbf{A}$ by $\mathbf{x} \stackrel{w}{\rightarrow} \mathbf{y}$ if there exists a run from $\mathbf{x}$ to $\mathbf{y}$ labeled by $w$. Given a language $W \subseteq \mathbf{A}^{*}$, we denote by $\stackrel{W}{\longrightarrow}$ the relation $\bigcup_{w \in W} \stackrel{w}{\longrightarrow}$. The relation $\stackrel{\mathbf{A}^{*}}{\longrightarrow}$ is called the reachability relation and it is denoted by $\stackrel{*}{\rightarrow}$. A subreachability relation is a relation included in $\stackrel{*}{\rightarrow}$.
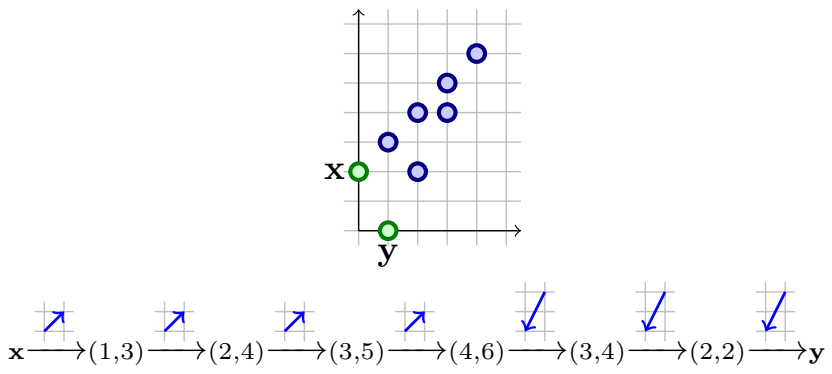

Figure 1. The run labeled by $(1,1)^{4}(-1,-2)^{3}$ with $\operatorname{dir}(\rho)=(\mathbf{x}, \mathbf{y})$.

Given a configuration $\mathbf{c} \in \mathbb{N}^{d}$ and a language $W \subseteq \mathbf{A}^{*}$ we denote by post $(\mathbf{c}, W)$ the set of configurations $\mathbf{y} \in \mathbb{N}^{d}$ such that $\mathbf{c} \stackrel{W}{\longrightarrow} \mathbf{y}$. Given a set of configurations $\mathbf{C} \subseteq \mathbb{N}^{d}$ and a language $W \subseteq\left(\mathbb{Z}^{d}\right)^{*}$ we denote by $\operatorname{post}(\mathbf{C}, W)$ the set of configurations $\bigcup_{\mathbf{c} \in \mathbf{C}} \operatorname{post}(\mathbf{c}, W)$. The set $\operatorname{post}\left(\mathbf{c}_{\text {init }}, \mathbf{A}^{*}\right)$ is called the reachability set. A subset of this set if called a subreachability set.

Flatability properties [11], [12] are defined thanks to bounded languages [13]. A language $W \subseteq \mathbf{A}^{*}$ is said to be bounded if there exists a finite sequence $w_{1}, \ldots w_{m}$ of words $w_{j} \in \mathbf{A}^{*}$ such that $W \subseteq w_{1}^{*} \ldots w_{m}^{*}$. Let us recall that bounded languages are stable by concatenation, union, intersection, and subset. A subreachability relation is said to be flatable if it is included in $\stackrel{W}{\longrightarrow}$ where $W \subseteq \mathbf{A}^{*}$ is a bounded language. A subreachability set is said to be flatable if it is included in $\operatorname{post}\left(\mathbf{c}_{\text {init }}, W\right)$ where $W \subseteq \mathbf{A}^{*}$ is a bounded language.

Definition III.1. A VAS is said to be flatable if its reachability set is flatable. A VAS is said to be Presburger if its reachability set is definable in the Presburger arithmetic.

In this paper we show that the class of Presburger VAS coincides with the class of flatable VAS. In the remainder of this section we recall elements of acceleration techniques that explain why flatable VAS are Presburger. We also explain why a Presburger formula denoting the reachability set is effectively computable in this case.

The displacement of a word $w=\mathbf{a}_{1} \ldots \mathbf{a}_{k}$ of actions $\mathbf{a}_{j} \in$ $\mathbf{A}$ is the vector $\Delta(w)=\sum_{j=1}^{k} \mathbf{a}_{j}$. Observe that $\mathbf{x} \stackrel{w}{\rightarrow} \mathbf{y}$ 
implies $\mathbf{x}+\Delta(w)=\mathbf{y}$ but the converse is not true in general. The converse property can be obtained by associating to every word $w=\mathbf{a}_{1} \ldots \mathbf{a}_{k}$ the configuration $\mathbf{c}_{w}$ defined for every $i \in\{1, \ldots, d\}$ by:

$$
\mathbf{c}_{w}(i)=\max \left\{-\left(\mathbf{a}_{1}+\cdots+\mathbf{a}_{j}\right)(i) \mid 0 \leq j \leq k\right\}
$$

The following lemma shows that $\mathbf{c}_{w}$ is the minimal for $\leq$ configuration from which there exists a run labeled by $w$.

Lemma III.2. There exists a run from a configuration $\mathbf{x} \in \mathbb{N}^{d}$ labeled by a word $w \in \mathbf{A}^{*}$ if, and only if, $\mathbf{x} \geq \mathbf{c}_{w}$.

Proof: We assume that $w=\mathbf{a}_{1} \ldots \mathbf{a}_{k}$ where $\mathbf{a}_{j} \in \mathbf{A}$. Assume first that there exists a run $\rho=\mathbf{c}_{0} \ldots \mathbf{c}_{k}$ labeled by $w$ from $\mathbf{c}_{0}=\mathbf{x}$. Since $\mathbf{a}_{j}=\mathbf{c}_{j}-\mathbf{c}_{j-1}$ we deduce that $\mathbf{c}_{j}=$ $\mathbf{x}+\mathbf{a}_{1}+\cdots+\mathbf{a}_{j}$. Since $\mathbf{c}_{j} \geq \mathbf{0}$ we get $\mathbf{x} \geq-\left(\mathbf{a}_{1}+\cdots+\mathbf{a}_{j}\right)$. We have proved that $\mathbf{x} \geq \mathbf{c}_{w}$. Conversely, let us assume that $\mathbf{x} \geq \mathbf{c}_{w}$ and let us prove that there exists a run from $\mathbf{x}$ labeled by $w$. We introduce the vectors $\mathbf{c}_{j}=\mathbf{x}+\mathbf{a}_{1}+\cdots+\mathbf{a}_{j}$. Since $\mathbf{x} \geq \mathbf{c}_{w}$ we deduce that $\mathbf{c}_{j} \in \mathbb{N}^{d}$. Therefore $\rho=\mathbf{c}_{0} \ldots \mathbf{c}_{k}$ is a run. Just observe that $\mathbf{c}_{0}=\mathbf{x}$ and $\rho$ is labeled by $w$.

The following lemma shows that the set of triples $(\mathbf{x}, n, \mathbf{y}) \in \mathbb{N}^{d} \times \mathbb{N} \times \mathbb{N}^{d}$ such that $\mathbf{x} \stackrel{w^{n}}{\longrightarrow} \mathbf{y}$ is effectively definable in the Presburger arithmetic. In particular with an existential quantification of the variable $n$, we deduce that the relation $\stackrel{w^{*}}{\longrightarrow}$ is effectively definable in the Presburger arithmetic. Hence if a set of configurations $\mathbf{C} \subseteq \mathbb{N}^{d}$ is denoted by a Presburger formula then for every word $w \in \mathbf{A}^{*}$ we can effectively compute a Presburger formula denoting $\operatorname{post}\left(\mathbf{C}, w^{*}\right)$.

Lemma III.3. A pair $(\mathbf{x}, \mathbf{y}) \in \mathbb{N}^{d} \times \mathbb{N}^{d}$ of configurations satisfies $\mathbf{x} \stackrel{w^{n}}{\longrightarrow} \mathbf{y}$ where $w \in \mathbf{A}^{*}$ and $n \in \mathbb{N}_{>0}$ if and only if:

$$
\mathbf{x} \geq \mathbf{c}_{w} \wedge \mathbf{x}+n \Delta(w)=\mathbf{y} \wedge \mathbf{y}-\Delta(w) \geq \mathbf{c}_{w}
$$

Proof: Assume first that we have a run $\mathbf{x} \stackrel{w^{n}}{\longrightarrow} \mathbf{y}$. Since $n \geq 1$, a prefix and a suffix of this run show that $\mathbf{x} \stackrel{w}{\rightarrow}$ $\mathbf{x}+\Delta(w)$ and $\mathbf{y}-\Delta(w) \stackrel{w}{\rightarrow} \mathbf{y}$. Lemma III.2 shows that $\mathbf{x} \geq \mathbf{c}_{w}$ and $\mathbf{y}-\Delta(w) \geq \mathbf{c}_{w}$. Moreover, since $\mathbf{x}+n \Delta(w)=\mathbf{y}$ we have proved one way of the lemma. For the other way, let us assume that $\mathbf{x} \geq \mathbf{c}_{w}, \mathbf{x}+n \Delta(w)=\mathbf{y}$, and $\mathbf{y}-\Delta(w) \geq \mathbf{c}_{w}$. We introduce the sequence $\mathbf{c}_{0}, \ldots, \mathbf{c}_{n}$ defined by $\mathbf{c}_{j}=\mathbf{x}+$ $j \Delta(w)$. Let us prove that $\mathbf{c}_{j-1} \geq \mathbf{c}_{w}$ for every $1 \leq j \leq n$. Let $i \in\{1, \ldots, d\}$. If $\Delta(w)(i) \geq 0$ then $\mathbf{c}_{j-1}(i) \geq \mathbf{x}(i) \geq$ $\mathbf{c}_{w}(i)$. Next, assume that $\Delta(w)(i)<0$. In this case, since $\mathbf{x}+n \Delta(w)=\mathbf{y}$ we deduce that $\mathbf{c}_{j-1}=\mathbf{y}-\Delta(w)+(n-$ $j)(-\Delta(w))$. Thus $\mathbf{c}_{j-1}(i) \geq \mathbf{y}(i)-\Delta(w)(i) \geq \mathbf{c}_{w}(i)$. We have proved that $\mathbf{c}_{j-1} \geq \mathbf{c}_{w}$. Lemma III.2 shows that $\mathbf{c}_{j-1} \stackrel{w}{\longrightarrow}$ $\mathbf{c}_{j}$. We have proved that $\mathbf{c}_{0} \stackrel{w^{n}}{\longrightarrow} \mathbf{c}_{n}$. Since $\mathbf{c}_{0}=\mathbf{x}$ and $\mathbf{c}_{n}=\mathbf{y}$ we have proved the other way.

We deduce the following theorem also proved in [16] in a more general context. This theorem shows that we can effectively compute a Presburger formula denoting the reachability set of flatable VAS.
Theorem III.4 ( [16]). There exists an algorithm computing for any flatable VAS $\left(\mathbf{c}_{\text {init }}, \mathbf{A}\right)$ a sequence $w_{1}, \ldots, w_{m} \in \mathbf{A}^{*}$ such that:

$$
\operatorname{post}\left(\mathbf{c}_{\text {init }}, \mathbf{A}^{*}\right)=\operatorname{post}\left(\mathbf{c}_{\text {init }}, w_{1}^{*} \ldots w_{m}^{*}\right)
$$

Proof: Let us consider an algorithm that takes as input a VAS $\left(\mathbf{c}_{\text {init }}, \mathbf{A}\right)$ and it computes inductively a sequence $\left(w_{m}\right)_{m \geq 1}$ of words $w_{m} \in \mathbf{A}^{*}$ such that every finite sequence $\left(\sigma_{j}\right)_{1 \leq j \leq n}$ of words $\sigma_{j} \in \mathbf{A}^{*}$ is a sub-sequence. Note that such an algorithm exists. From this sequence, another algorithm computes inductively Presburger formulas denoting sets of configurations $\mathbf{C}_{m} \subseteq \mathbb{N}^{d}$ satisfying $\mathbf{C}_{0}=\left\{\mathbf{c}_{\text {init }}\right\}$ and $\mathbf{C}_{m}=\operatorname{post}\left(\mathbf{C}_{m-1}, w_{m}^{*}\right)$ for every $m \in \mathbb{N}_{>0}$. The algorithm stops and it returns $w_{1}, \ldots, w_{m}$ when $\operatorname{post}\left(\mathbf{C}_{m}, \mathbf{A}\right) \subseteq \mathbf{C}_{m}$. Note that such a test is implementable since $\mathbf{C}_{m}$ is denoted by a Presburger formula and the Presburger arithmetic is a decidable logic. When the algorithm stops the set $\mathbf{C}_{m}$ is included in the reachability set and it satisfies post $\left(\mathbf{C}_{m}, \mathbf{A}\right) \subseteq \mathbf{C}_{m}$. We deduce that $\mathbf{C}_{m}$ is equal to the reachability set. In particular the reachability set if equal to $\operatorname{post}\left(\mathbf{c}_{\text {init }}, w_{1}^{*} \ldots w_{m}^{*}\right)$ and the algorithm is correct.

For the termination, since the VAS is flatable, there exists a bounded language $W \subseteq \mathbf{A}^{*}$ such that the reachability set is included in $\operatorname{post}\left(\mathbf{c}_{\text {init }}, W\right)$. As $W$ is bounded, there exists a finite sequence $\sigma_{1}, \ldots, \sigma_{n} \in \mathbf{A}^{*}$ such that $W \subseteq \sigma_{1}^{*} \ldots \sigma_{n}^{*}$. There exists $m \in \mathbb{N}$ such that this sequence is a sub-sequence of $w_{1}, \ldots, w_{m}$. Let us observe that $W \subseteq \sigma_{1}^{*} \ldots \sigma_{n}^{*} \subseteq w_{1}^{*} \ldots w_{m}^{*}$. From the following inclusions we deduce that $\mathbf{C}_{m}$ is equal to the reachability set:

$$
\begin{aligned}
\operatorname{post}\left(\mathbf{c}_{\text {init }}, \mathbf{A}^{*}\right) & \subseteq \operatorname{post}\left(\mathbf{c}_{\text {init }}, W\right) \\
& \subseteq \operatorname{post}\left(\mathbf{c}_{\text {init }}, w_{1}^{*} \ldots w_{m}^{*}\right) \\
& =\mathbf{C}_{m} \\
& \subseteq \operatorname{post}\left(\mathbf{c}_{\text {init }}, \mathbf{A}^{*}\right)
\end{aligned}
$$

In particular post $\left(\mathbf{C}_{m}, \mathbf{A}\right) \subseteq \mathbf{C}_{m}$ and the algorithm terminates before the $m$ th iteration.

Corollary III.5. Reachability sets of flatable VAS are effectively definable in the Presburger arithmetic.

In the remainder of this paper, we proved that Presburger VAS are flatable. As a direct consequence a Presburger formula denoting the reachability set of a Presburger VAS is effectively computable using classical acceleration techniques.

\section{WELL-PREORDERS}

A relation $R$ over a set $S$ is a subset $R \subseteq S \times S$. The composition of two relations $R_{1}, R_{2}$ over $S$ is the relation over $S$ denoted by $R_{1} \circ R_{2}$ and defined as the set $\bigcup_{i \in S}\{(s, t) \in$ $\left.S \times S \mid(s, i) \in R_{1} \wedge(i, t) \in R_{2}\right\}$. A relation $R$ over $S$ is said to be reflexive if $(s, s) \in R$ for every $s \in S$, transitive if $R \circ R \subseteq R$, antisymmetric if $(s, t),(t, s) \in R$ implies $s=t$, a preorder if $R$ is reflexive and transitive, and an order if $R$ is an antisymmetric preorder. The composition of $R$ by itself $n$ 
times where $n \in \mathbb{N}_{>0}$ is denoted by $R^{n}$. The transitive closure of a relation $R$ is the relation $\bigcup_{n>1} R^{n}$ denoted by $R^{+}$.

A preorder $\sqsubseteq$ over a set $S$ is said to be well if for every sequence $\left(s_{n}\right)_{n \in \mathbb{N}}$ of elements $s_{n} \in S$ there exists an infinite set $N \subseteq \mathbb{N}$ such that $s_{n} \sqsubseteq s_{m}$ for every $n \leq m$ in $N$. Observe that $(\mathbb{N}, \leq)$ is a well-ordered set whereas $(\mathbb{Z}, \leq)$ is not wellordered. As another example, the pigeonhole principle shows that a set $S$ is well-ordered by the equality relation if, and only if, $S$ is finite. Well-preorders can be easily defined thanks to Dickson's lemma and Higman's lemma as follows.

Dickson's lemma: Dickson's lemma shows that the Cartesian product of two well-preordered sets is well-preordered. More formally, given two preordered sets $\left(S_{1}, \sqsubseteq_{1}\right)$ and $\left(S_{2}, \sqsubseteq_{2}\right)$ we denote by $\sqsubseteq_{1} \times \sqsubseteq_{2}$ the preorder defined component-wise over the Cartesian product $S_{1} \times S_{2}$ by $\left(s_{1}, s_{2}\right) \sqsubseteq_{1} \times \sqsubseteq_{2}\left(s_{1}^{\prime}, s_{2}^{\prime}\right)$ if $s_{1} \sqsubseteq_{1} s_{1}^{\prime}$ and $s_{2} \sqsubseteq_{2} s_{2}^{\prime}$. Dickson's lemma says that $\left(S_{1} \times S_{2}, \sqsubseteq_{1} \times \sqsubseteq_{2}\right)$ is well-preordered for every well-preordered sets $\left(S_{1}, \sqsubseteq_{1}\right)$ and $\left(S_{2}, \sqsubseteq_{2}\right)$. As a direct application, the set $\mathbb{N}^{d}$ equipped with the component-wise extension of $\leq$ is well-ordered.

Higman's lemma: Higman's lemma shows that words over well-preordered alphabets can be well-preordered. More formally, given a preordered set $(S, \sqsubseteq)$, we introduce the set $S^{*}$ of words over $S$ equipped with the preorder $\sqsubseteq^{*}$ defined by $w \sqsubseteq^{*} w^{\prime}$ if $w$ and $w^{\prime}$ can be decomposed into $w=s_{1} \ldots s_{k}$ and $w^{\prime} \in S^{*} s_{1}^{\prime} S^{*} \ldots s_{k}^{\prime} S^{*}$ where $s_{j} \sqsubseteq s_{j}^{\prime}$ are in $S$ for every $j \in\{1, \ldots, k\}$. Higman's lemma says that $\left(S^{*}, \sqsubseteq^{*}\right)$ is wellpreordered for every well-preordered set $(S, \sqsubseteq)$. As a classical application, the set of words over a finite alphabet is wellordered by the sub-word relation.

\section{Vector Spaces, Conic Sets, Periodic Sets, And LATTICES}

In this section we recall some elements of linear algebra. We also introduce the central notions of definable conic sets and smooth periodic sets.

A vector space is a set $\mathbf{V} \subseteq \mathbb{Q}^{d}$ such that $\mathbf{0} \in \mathbf{V}, \mathbf{V}+\mathbf{V} \subseteq$ $\mathbf{V}$, and $\mathbb{Q} \mathbf{V} \subseteq \mathbf{V}$. Any set $\mathbf{X} \subseteq \mathbb{Q}^{d}$ is included in a unique minimal under set inclusion vector space. This vector space called the vector space generated by $\mathbf{X} \subseteq \mathbb{Q}^{d}$. Let us recall that every vector space $\mathbf{V}$ is generated by a finite set. The rank $\operatorname{rank}(\mathbf{V})$ of a vector space $\mathbf{V}$ is the minimal natural number $r \in \mathbb{N}$ such that there exists a finite set $\mathbf{B}$ with $r$ vectors that generates $\mathbf{V}$. Let us recall that $\operatorname{rank}(\mathbf{V}) \leq \operatorname{rank}(\mathbf{W})$ for every pair of vector spaces $\mathbf{V} \subseteq \mathbf{W}$. Moreover, if $\mathbf{V}$ is strictly included in $\mathbf{W}$ then $\operatorname{rank}(\mathbf{V})<\operatorname{rank}(\mathbf{W})$. Vectors spaces are geometrically characterized as follows:

Lemma V.1 ( [17]). A set $\mathbf{V} \subseteq \mathbb{Q}^{d}$ is a vector space if and only if there exists a finite set $\mathbf{H} \subseteq \mathbb{Q}^{d}$ such that:

$$
\mathbf{V}=\left\{\mathbf{v} \in \mathbb{Q}^{d} \mid \bigwedge_{\mathbf{h} \in \mathbf{H}} \mathbf{h} \cdot \mathbf{v}=0\right\}
$$
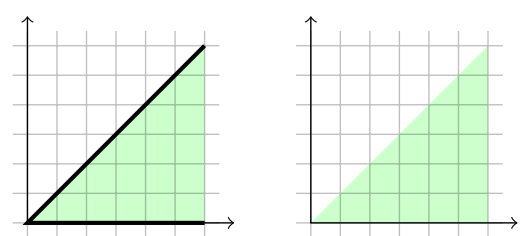

Figure 2. The finitely generated conic set $\mathbb{Q}_{>0}(1,1)+\mathbb{Q}_{>0}(1,0)$ and the definable conic set $\{(0,0)\} \cup\left\{\left(c_{1}, c_{2}\right) \in \mathbb{Q}_{>0}^{2} \mid c_{2}<c_{1}\right\}$

A conic set is a set $\mathbf{C} \subseteq \mathbb{Q}^{d}$ such that $\mathbf{0} \in \mathbf{C}, \mathbf{C}+\mathbf{C} \subseteq \mathbf{C}$ and $\mathbb{Q}>0$ C $\subseteq \mathbf{C}$. Any set $\mathbf{X} \subseteq \mathbb{Q}^{d}$ is included in a unique minimal under set inclusion conic set. This conic set is called the conic set generated by $\mathbf{X} \subseteq \mathbb{Q}^{d}$. Contrary to the vector spaces, some conic sets are not finitely generated. Finitely generated conic sets are geometrically characterized by the following lemma.

Lemma V.2 ( [17]). A set $\mathbf{C} \subseteq \mathbb{Q}^{d}$ is a finitely generated conic set if and only if there exists a finite set $\mathbf{H} \subseteq \mathbb{Q}^{d}$ such that:

$$
\mathbf{C}=\left\{\mathbf{c} \in \mathbb{Q}^{d} \mid \bigwedge_{\mathbf{h} \in \mathbf{H}} \mathbf{h} \cdot \mathbf{c} \geq 0\right\}
$$

Definition V.3. A conic set is said to be definable (polytope in [18]) if it can be defined by a formula in $\mathrm{FO}(\mathbb{Q},+, \leq)$.

Example V.4. The conic set $\mathbf{C}=\left\{\left(c_{1}, c_{2}\right) \in \mathbb{Q} \times \mathbb{Q} \mid c_{1} \leq\right.$ $\left.\sqrt{2} c_{2}\right\}$ is not definable. Fig. 2 depicts a finitely generated conic set and a definable conic set which is not finitely generated.

A periodic set is a set $\mathbf{P} \subseteq \mathbb{Q}^{d}$ such that $\mathbf{0} \in \mathbf{P}$, and $\mathbf{P}+\mathbf{P} \subseteq \mathbf{P}$. Any set $\mathbf{X} \subseteq \mathbb{Q}^{d}$ is included in a unique minimal under set inclusion periodic set. This periodic set is called the periodic set generated by $\mathbf{X}$. Observe that the conic set $\mathbf{C}$ generated by a periodic set $\mathbf{P}$ is $\mathbf{C}=\mathbb{Q}_{\geq 0} \mathbf{P}$. The finitely generated periodic sets are characterized as follows. Given a periodic set $\mathbf{P}$ we denote by $\leq_{\mathbf{P}}$ the preorder over $\mathbf{P}$ defined by $\mathbf{p} \leq_{\mathbf{P}} \mathbf{q}$ if $\mathbf{q} \in \mathbf{p}+\mathbf{P}$. A periodic set $\mathbf{P} \subseteq \mathbb{Q}^{d}$ is said to be discrete if there exists $n \in \mathbb{N}_{>0}$ such that $\mathbf{P} \subseteq \frac{1}{n} \mathbb{Z}^{d}$. Observe that finitely generated periodic sets are discrete. The following lemma characterizes the discrete periodic sets that are finitely generated. The proof is given in appendix.

Lemma V.5. Let $\mathbf{P}$ be a discrete periodic set. The following conditions are equivalent:

- $\mathbf{P}$ is finitely generated as a periodic set.

- $\left(\mathbf{P}, \leq_{\mathbf{P}}\right)$ is well-preordered.

- $\mathbb{Q}_{\geq 0} \mathbf{P}$ is finitely generated as a conic set.

Remark V.6. A set $\mathbf{X} \subseteq \mathbb{Z}^{d}$ is definable in the Presburger arithmetic $\mathrm{FO}(\mathbb{Z},+, \leq)$ if, and only if, it is a finite union of linear sets $\mathbf{b}+\mathbf{P}$ where $\mathbf{b} \in \mathbb{Z}^{d}$ and $\mathbf{P} \subseteq \mathbb{Z}^{d}$ is a finitely generated periodic set [8].

A limit of a periodic set $\mathbf{P} \subseteq \mathbb{Q}^{d}$ is a vector $\mathbf{v} \in \mathbb{Q}^{d}$ such that there exists $\mathbf{p} \in \mathbf{P}$ and $n \in \mathbb{N}_{>0}$ satisfying $\mathbf{p}+n \mathbb{N} \mathbf{v} \subseteq \mathbf{P}$. The set of limits of $\mathbf{P}$ is denoted by $\lim (\mathbf{P})$. 
Lemma V.7. $\lim (\mathbf{P})$ is a conic set.

Proof: Let $\mathbf{C}=\lim (\mathbf{P})$. Let $\mathbf{v}_{1}, \mathbf{v}_{2} \in \mathbf{C}$. There exist $\mathbf{p}_{1}, \mathbf{p}_{2} \in \mathbf{P}$ and $n_{1}, n_{2} \in \mathbb{N}_{>0}$ such that $\mathbf{p}_{1}+n_{1} \mathbb{N}_{1}$ and $\mathbf{p}_{2}+n_{2} \mathbb{N v}_{2}$ are included in $\mathbf{P}$. Let $n=n_{1} n_{2}$. Since $n \mathbb{N}$ is included in $n_{1} \mathbb{N}$ and $n_{2} \mathbb{N}$ we deduce that $\mathbf{p}_{1}+n \mathbb{N} \mathbf{v}_{1}$ and $\mathbf{p}_{2}+n \mathbb{N v}_{2}$ are included in $\mathbf{P}$. As $\mathbf{P}$ is periodic we deduce that $\mathbf{p}+n \mathbb{N} \mathbf{v} \subseteq \mathbf{P}$ where $\mathbf{p}=\mathbf{p}_{1}+\mathbf{p}_{2}$ and $\mathbf{v}=\mathbf{v}_{1}+\mathbf{v}_{2}$. As $\mathbf{p} \in \mathbf{P}$ we get $\mathbf{v} \in \mathbf{C}$. We deduce that $\mathbf{C}+\mathbf{C} \subseteq \mathbf{C}$. Since $\mathbf{0} \in \mathbf{C}$ and $\mathbb{Q}_{\geq 0} \mathbf{C} \subseteq \mathbf{C}$ are immediate, we have proved that $\mathrm{C}$ is a conic set.

A periodic set $\mathbf{P}$ is said to be well-limit if for every sequence $\left(\mathbf{p}_{n}\right)_{n \in \mathbb{N}}$ of vectors $\mathbf{p}_{n} \in \mathbf{P}$ there exists an infinite set $N \subseteq \mathbb{N}$ such that $\mathbf{p}_{m}-\mathbf{p}_{n} \in \lim (\mathbf{P})$ for every $n \leq m$ in $N$. The periodic set $\mathbf{P}$ is said to be smooth if $\lim (\mathbf{P})$ is a definable conic set and $\mathbf{P}$ is well-limit.

Example V.8. Let us consider the periodic set $\mathbf{P} \subseteq \mathbb{N}^{2}$ generated by $(0,1)$ and the pairs $\left(2^{m}, 1\right)$ where $m \in \mathbb{N}$. The limit of $\mathbf{P}$ is the definable conic set $\mathbf{C}=\{(0,0)\} \cup(\mathbb{Q} \geq 0 \times \mathbb{Q}>0)$. Note that $\mathbf{P}$ is not well-limit since the sequence $\left(\mathbf{p}_{n}\right)_{n \in \mathbb{N}}$ defined by $\mathbf{p}_{n}=\left(2^{n}, 1\right)$ is such that $\mathbf{p}_{m}-\mathbf{p}_{n}=\left(2^{m}-2^{n}, 0\right) \notin \mathbf{C}$ for every $n<m$.

Example V.9. The periodic set $\mathbf{P}=\{(0,0)\} \cup\left(\mathbb{N}_{>0} \times \mathbb{N}_{>0}\right)$ is smooth and $\lim (\mathbf{P})=\mathbb{Q}_{\geq 0} \times \mathbb{Q}_{\geq 0}$.

A lattice is a set $\mathbf{L} \subseteq \mathbb{Q}^{d}$ such that $\mathbf{0} \in \mathbf{L}, \mathbf{L}+\mathbf{L} \subseteq \mathbf{L}$ and $-\mathbf{L} \subseteq \mathbf{L}$. Any set $\mathbf{X} \subseteq \mathbb{Q}^{d}$ is included in a unique minimal under set inclusion lattice. This lattice is called the lattice generated by $\mathbf{X}$. Observe that the conic set generated by a lattice $\mathbf{L}$ is equal to the vector space $\mathbf{V}=\mathbb{Q}_{\geq 0} \mathbf{L}$. Since vector spaces are finitely generated, the previous Lemma V.5 shows that discrete lattices are finitely generated (as periodic sets and in particular as lattices).

Remark V.10. The following relations hold:

\begin{tabular}{|c|c|c|c|c|c|c|}
\hline $\begin{array}{l}\text { conic } \\
\text { sets }\end{array}$ & $\subset$ & $\begin{array}{c}\text { periodic } \\
\text { sets }\end{array}$ & $\supset$ & $\begin{array}{c}\text { discrete } \\
\text { periodic } \\
\text { sets }\end{array}$ & ว & $\begin{array}{c}\text { finitely gen. } \\
\text { periodic } \\
\text { sets }\end{array}$ \\
\hline$U$ & & $U$ & & U & & $\cup$ \\
\hline $\begin{array}{l}\text { vector } \\
\text { spaces }\end{array}$ & $\subset$ & lattices & $\supset$ & $\begin{array}{l}\text { discrete } \\
\text { lattices }\end{array}$ & - & $\begin{array}{c}\text { finitely gen. } \\
\text { lattices }\end{array}$ \\
\hline
\end{tabular}

Example V.11. The following periodic sets provide the strictness of the previous inclusion relations : $\mathbb{N}, \mathbb{Z}, \mathbb{Q}, \mathbb{Q} \geq 0$, $\{(x, y) \in \mathbb{Q} \times \mathbb{Q} \mid x \leq \sqrt{2} y\}$.

\section{Well-Order Over The Runs}

We define a well-order over the runs as follows. We introduce the relation $\unlhd$ over the runs defined by $\rho \unlhd \rho^{\prime}$ if $\rho$ is a run of the form $\rho=\mathbf{c}_{0} \ldots \mathbf{c}_{k}$ where $\mathbf{c}_{j} \in \mathbb{N}^{d}$ and if there exists a sequence $\left(\mathbf{v}_{j}\right)_{0 \leq j \leq k+1}$ of vectors $\mathbf{v}_{j} \in \mathbb{N}^{d}$ such that $\rho^{\prime}$ is a run of the form $\rho^{\prime}=\rho_{0} \ldots \rho_{k}$ where $\rho_{j}$ is a run from $\mathbf{c}_{j}+\mathbf{v}_{j}$ to $\mathbf{c}_{j}+\mathbf{v}_{j+1}$.

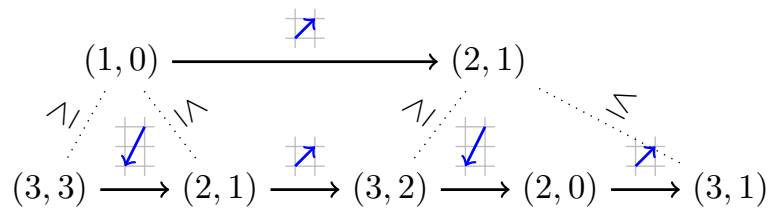

Figure 3. $(1,0)(2,1) \unlhd(3,3)(2,1)(3,2)(2,0)(3,1)$

Example VI.1. This example is depicted on Figure 3. Let $\rho=$ $(1,0)(2,1)$ and observe that $\rho \unlhd \rho_{1} \rho_{2}$ where $\rho_{1}=(3,3)(2,1)$ and $\rho_{2}=(3,2)(2,0)(3,1)$.

Let us recall the following lemma based on the Higman's Lemma.

Lemma VI.2 ( [14], [19]). The relation $\unlhd$ is a well-order.

Lemma VI.3. For every pair of runs $\rho \unlhd \rho^{\prime}$, the pair $(\mathbf{e}, \mathbf{f})=\operatorname{dir}\left(\rho^{\prime}\right)-\operatorname{dir}(\rho)$ satisfies $\operatorname{dir}(\rho)+\mathbb{N}(\mathbf{e}, \mathbf{f})$ is a flatable subreachability relation.

Proof: Assume that $\rho \unlhd \rho^{\prime}$. In this case $\rho=\mathbf{c}_{0} \ldots \mathbf{c}_{k}$ where $\mathbf{c}_{j} \in \mathbb{N}^{d}$ and there exists a sequence $\mathbf{v}_{0}, \ldots, \mathbf{v}_{k+1} \in \mathbb{N}^{d}$ such that $\rho^{\prime}=\rho_{0} \ldots \rho_{k}$ where $\rho_{j}$ is a run from $\mathbf{c}_{j}+\mathbf{v}_{j}$ to $\mathbf{c}_{j}+\mathbf{v}_{j+1}$ labeled by a word $\sigma_{j}$. We introduce the actions $\mathbf{a}_{1}, \ldots, \mathbf{a}_{k}$ defined by $\mathbf{a}_{j}=\mathbf{c}_{j}-\mathbf{c}_{j-1}$. By monotony we deduce that for every $r \in \mathbb{N}$ we have a run from $\mathbf{c}_{j}+r \mathbf{v}_{j}$ to $\mathbf{c}_{j}+r \mathbf{v}_{j+1}$ labeled by $\sigma_{j}^{r}$. We also have $\mathbf{c}_{j}+r \mathbf{v}_{j+1} \stackrel{\mathbf{a}_{j}}{\longrightarrow}$ $\mathbf{c}_{j+1}+r \mathbf{v}_{j+1}$. We obtain from these runs, a run $\rho_{r}$ from $\mathbf{c}_{0}+r \mathbf{v}_{0}$ to $\mathbf{c}_{k}+r \mathbf{v}_{k+1}$ labeled by $\sigma_{0}^{r} \mathbf{a}_{1} \sigma_{1}^{r} \ldots \mathbf{a}_{k} \sigma_{k}^{r}$. Since $(\mathbf{e}, \mathbf{f})=\operatorname{dir}\left(\rho^{\prime}\right)-\operatorname{dir}(\rho)$ is the pair $\left(\mathbf{v}_{0}, \mathbf{v}_{k+1}\right)$ we deduce that $\operatorname{dir}(\rho)+\mathbb{N}(\mathbf{e}, \mathbf{f})$ is included in $\stackrel{W}{\longrightarrow}$ where $W=$ $\sigma_{0}^{*} \mathbf{a}_{1} \sigma_{1}^{*} \ldots \mathbf{a}_{k} \sigma_{k}^{*}$.

Based on the definition of the well-order $\unlhd$, we introduce the transformer relation with capacity $\mathbf{c} \in \mathbb{N}^{d}$ as the relation $\stackrel{\mathrm{c}}{\curvearrowright}$ over $\mathbb{N}^{d}$ defined by $\mathbf{x} \stackrel{\mathbf{c}}{\curvearrowright} \mathbf{y}$ if there exists a run from $\mathbf{c}+\mathbf{x}$ to $\mathbf{c}+\mathbf{y}$. By monotony, let us observe that $\stackrel{\mathrm{c}}{\curvearrowright}$ is a periodic relation.

Remark VI.4. In [19], the conic relation $\mathbb{Q}_{\geq 0} \stackrel{\mathrm{c}}{\curvearrowright}$ is shown to be definable.

\section{Reflexive Definable Conic Relations}

The class of finite unions of reflexive definable conic relations over $\mathbb{Q}_{\geq 0}^{d}$ are clearly stable by composition, sum, intersection, and union. In the appendix, the following theorem is proved:

Theorem VII.1. Transitive closures of finite unions of reflexive definable conic relations over $\mathbb{Q}_{\geq 0}^{d}$ are reflexive definable conic relations.

Example VII.2. Let us consider the reflexive definable conic relation $R=\left\{\left(x, x^{\prime}\right) \in \mathbb{Q}_{\geq 0}^{2} \mid x \leq x^{\prime} \leq 2 x\right\}$. Observe that $R^{n}$ where $n \geq 1$ is the reflexive definable conic relation $\left\{\left(x, x^{\prime}\right) \in \mathbb{Q}_{\geq 0}^{2} \mid x \leq x^{\prime} \leq 2^{n} x\right\}$. Thus $R^{+}=\{(0,0)\} \cup$ $\left\{\left(x, x^{\prime}\right) \mid 0<x \leq x^{\prime}\right\}$. Observe that $R^{n}$ is strictly included 
in $R^{+}$for every $n \geq 1$. Hence $R^{+}$cannot be computed with a finite Kleene iteration $R^{1} \cup \ldots \cup R^{n}$.

\section{TRANSFORMER RELATIONS}

In this section, we prove the following theorem. All other results are not used in the sequel.

Theorem VIII.1. For every capacity $\mathbf{c} \in \mathbb{N}^{d}$ and for every periodic relation $P$ included in $\stackrel{\mathrm{c}}{\curvearrowright}$, there exists a definable conic relation $R \subseteq \mathbb{Q}_{\geq 0}^{d} \times \mathbb{Q}_{\geq 0}^{d}$ such that $\lim (P) \subseteq R$ and such that for every $(\mathbf{e}, \mathbf{f}) \in \bar{R}$ there exists $(\mathbf{x}, \mathbf{y}) \in P$ and $n \in \mathbb{N}_{>0}$ such that

$$
(\mathbf{c}, \mathbf{c})+(\mathbf{x}, \mathbf{y})+n \mathbb{N}(\mathbf{e}, \mathbf{f})
$$

is a flatable subreachability relation.

Theorem VIII.1 is obtained by following the approach introduced in [19]. Note that even if some lemmas are very similar to the ones given in that paper, proofs must be adapted to our context. In this new context, Theorem VII.1 is central for proving the existence of the relation $R$ introduced by Theorem VIII.1 (introduced as $R_{\gamma}$ in the sequel).

In the remainder of this section, $\gamma$ denotes a pair $(\mathbf{c}, P)$ where $\mathbf{c} \in \mathbb{N}^{d}$ is a capacity, and $P \subseteq \stackrel{\mathrm{c}}{\curvearrowright}$ is a periodic relation. We introduce the set $\Omega_{\gamma}$ of runs $\rho$ such that $\operatorname{dir}(\rho) \in(\mathbf{c}, \mathbf{c})+P$. Note that $\Omega_{\gamma}$ is non empty since it contains the run reduced to the single configuration $\mathbf{c}$. We denote by $\mathbf{Q}_{\gamma}$ the set of configurations $\mathbf{q} \in \mathbb{N}^{d}$ such that there exists a run $\rho \in \Omega_{\gamma}$ in which q occurs. We denote by $I_{\gamma}$ the set of indexes $i \in$ $\{1, \ldots, d\}$ such that $\left\{\mathbf{q}(i) \mid \mathbf{q} \in \mathbf{Q}_{\gamma}\right\}$ is finite. We consider the projection function $\pi_{\gamma}: \mathbf{Q}_{\gamma} \rightarrow \mathbb{N}^{I_{\gamma}}$ defined by $\pi_{\gamma}(\mathbf{q})(i)=$ $\mathbf{q}(i)$. We introduce the finite set of states $S_{\gamma}=\pi_{\gamma}\left(\mathbf{Q}_{\gamma}\right)$ and the set $T_{\gamma}$ of transitions $\left(\pi_{\gamma}(\mathbf{q}), \mathbf{q}^{\prime}-\mathbf{q}, \pi_{\gamma}\left(\mathbf{q}^{\prime}\right)\right)$ where $\mathbf{q q}^{\prime}$ is a factor of a run in $\Omega_{\gamma}$. We introduce $s_{\gamma}=\pi_{\gamma}(\mathbf{c})$. Since $T_{\gamma} \subseteq S_{\gamma} \times \mathbf{A} \times S_{\gamma}$ we deduce that $T_{\gamma}$ is finite. We introduce the graph $G_{\gamma}=\left(S_{\gamma}, T_{\gamma}\right)$.
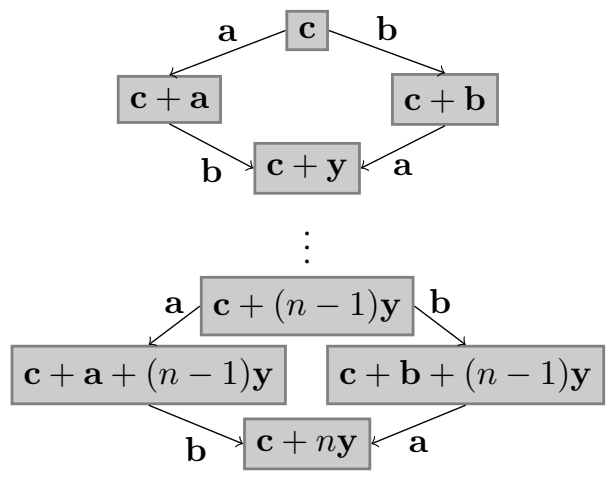

Figure 4. Set of runs $\Omega_{\gamma}$.

Example VIII.2. Let us consider the VAS $\mathbf{A}=\{\mathbf{a}, \mathbf{b}\}$ where $\mathbf{a}=(1,1,-1)$ and $\mathbf{b}=(-1,0,1)$, and let us consider the pair $(\mathbf{c}, P)$ where $\mathbf{c}=(1,0,1)$, and $P=\mathbb{N}(\mathbf{0}, \mathbf{y})$ with $\mathbf{y}=$

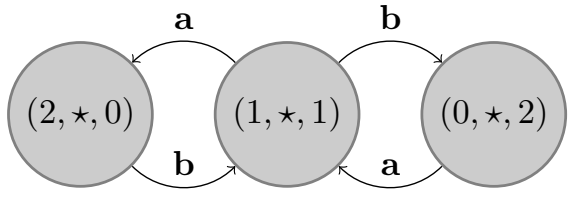

Figure 5. Graph $G_{\gamma}$.

$(0,1,0)$. Note that $P$ is included in $\stackrel{\mathrm{c}}{\curvearrowright}$ since there exists a run $\mathbf{c} \stackrel{(\mathbf{a b})^{n}}{\longrightarrow} \mathbf{c}+n \mathbf{y}$ for every $n \in \mathbb{N}$. The set $\Omega_{\gamma}$ is depicted in Figure 4. This set is equal to $\left\{\mathbf{c} \stackrel{w_{1} \ldots w_{n}}{\longrightarrow} \mathbf{c}+n \mathbf{y} \mid n \in\right.$ $\left.\mathbb{N} w_{j} \in\{\mathbf{a b}, \mathbf{b a}\}\right\}$. Observe that $\mathbf{Q}_{\gamma}=(\mathbf{c}+\mathbf{a}+\mathbb{N} \mathbf{y}) \cup(\mathbf{c}+$ $\mathbb{N y}) \cup(\mathbf{c}+\mathbf{b}+\mathbb{N y})$. Hence the set of bounded components is $I_{\gamma}=\{1,3\}$. Observe that $\pi_{\gamma}(\mathbf{c}+\mathbf{a}+n \mathbf{y})=(2, \star, 0)$, $\pi_{\gamma}(\mathbf{c}+n \mathbf{y})=(1, \star, 1)$, and $\pi_{\gamma}(\mathbf{c}+\mathbf{b}+n \mathbf{y})=(0, \star, 2)$ where $\star$ denotes a projected component. Hence $s_{\gamma}=(1, \star, 1)$ and $S_{\gamma}=\{(2, \star, 0),(1, \star, 1),(0, \star, 2)\}$. The graph $G_{\gamma}$ is depicted on Figure 5.

An intraproduction for $\gamma$ is a vector $\mathbf{h} \in \mathbb{N}^{d}$ such that $\mathbf{c}+\mathbf{h} \in \mathbf{Q}_{\gamma}$. We denote by $\mathbf{H}_{\gamma}$ the set of intraproduction for $\gamma$. The following Lemma VIII.3 shows that this set is periodic. In particular for every $\mathbf{h} \in \mathbf{H}_{\gamma}$, from $\mathbf{c}+\mathbb{N} \mathbf{h} \subseteq \mathbf{Q}_{\gamma}$ we deduce that $\mathbf{h}(i)=0$ for every $i \in I_{\gamma}$.

Lemma VIII.3. We have $\mathbf{Q}_{\gamma}+\mathbf{H}_{\gamma} \subseteq \mathbf{Q}_{\gamma}$.

Proof: Let $\mathbf{q} \in \mathbf{Q}_{\gamma}$ and $\mathbf{h} \in \mathbf{H}_{\gamma}$. As $\mathbf{q} \in \mathbf{Q}_{\gamma}$, there exist $(\mathbf{x}, \mathbf{y}) \in P$ and words $u, v \in \mathbf{A}^{*}$ such that $\mathbf{c}+\mathbf{x} \stackrel{u}{\rightarrow} \mathbf{q} \stackrel{v}{\rightarrow} \mathbf{c}+\mathbf{y}$. Since $\mathbf{h} \in \mathbf{H}_{\gamma}$ there exist $\left(\mathbf{x}^{\prime}, \mathbf{y}^{\prime}\right) \in P$ and words $u^{\prime}, v^{\prime} \in \mathbf{A}^{*}$ such that $\mathbf{c}+\mathbf{x}^{\prime} \stackrel{u^{\prime}}{\longrightarrow} \mathbf{c}+\mathbf{h} \stackrel{v^{\prime}}{\longrightarrow} \mathbf{c}+\mathbf{y}^{\prime}$. By monotony, we have $\mathbf{c}+\left(\mathbf{x}+\mathbf{x}^{\prime}\right) \stackrel{u^{\prime} u}{\longrightarrow} \mathbf{q}+\mathbf{h} \stackrel{v v^{\prime}}{\longrightarrow} \mathbf{c}+\left(\mathbf{y}+\mathbf{y}^{\prime}\right)$. As $P$ is periodic, we deduce that $\mathbf{q}+\mathbf{h} \in \mathbf{Q}_{\gamma}$.

Example VIII.4. Let us come back to Example VIII.2. Note that $\mathbf{H}_{\gamma}=\mathbb{N} \mathbf{y}$. Observe that $\mathbf{Q}_{\gamma}+\mathbf{H}_{\gamma}=\mathbf{Q}_{\gamma}$.

Corollary VIII.5. We have $\pi_{\gamma}(\operatorname{src}(\rho))=s_{\gamma}=\pi_{\gamma}(\operatorname{tgt}(\rho))$ for every run $\rho \in \Omega_{\gamma}$.

Proof: Since $\rho \in \Omega_{\gamma}$ there exists $(\mathbf{x}, \mathbf{y}) \in P$ such that $\rho$ is a run from $\mathbf{c}+\mathbf{x}$ to $\mathbf{c}+\mathbf{y}$. In particular $\mathbf{x}$ and $\mathbf{y}$ are two intraproductions for $\gamma$. We deduce that $\mathbf{x}(i)=0=\mathbf{y}(i)$ for every $i \in I_{\gamma}$. Hence $\pi_{\gamma}(\operatorname{src}(\rho))=\pi_{\gamma}(\mathbf{c})=\pi_{\gamma}(\operatorname{tgt}(\rho))$.

A path in $G_{\gamma}$ is a word $p=\left(s_{0}, \mathbf{a}_{1}, s_{1}\right) \ldots\left(s_{k-1}, \mathbf{a}_{k}, s_{k}\right)$ of transitions $\left(s_{j-1}, \mathbf{a}_{j}, s_{j}\right)$ in $T_{\gamma}$. Such a path is called a path from $s_{0}$ to $s_{k}$ labeled by $w=\mathbf{a}_{1} \ldots \mathbf{a}_{k}$. When $s_{0}=s_{k}$ the path is called a cycle. The previous corollary shows that every run $\rho=\mathbf{c}_{0} \ldots \mathbf{c}_{k}$ in $\Omega_{\gamma}$ labeled by a word $w=\mathbf{a}_{1} \ldots \mathbf{a}_{k}$ provides the cycle $t_{1} \ldots t_{k}$ in $G_{\gamma}$ on $s_{\gamma}$ labeled by $w$ where $t_{j}=\left(\pi_{\gamma}\left(\mathbf{c}_{j-1}\right), \mathbf{a}_{j}, \pi_{\gamma}\left(\mathbf{c}_{j}\right)\right)$. We deduce that $G_{\gamma}$ is strongly connected.

Lemma VIII.6. For every $\mathbf{q} \leq \mathbf{q}^{\prime}$ in $\mathbf{Q}_{\gamma}$ there exists an intraproduction $\mathbf{h} \in \mathbf{H}_{\gamma}$ such that $\mathbf{q}^{\prime} \leq \mathbf{q}+\mathbf{h}$.

Proof: As $\mathbf{q}, \mathbf{q}^{\prime} \in \mathbf{Q}_{\gamma}$ there exist $(\mathbf{x}, \mathbf{y}),\left(\mathbf{x}^{\prime}, \mathbf{y}^{\prime}\right) \in P$, 
and there exist $u, v, u^{\prime}, v^{\prime} \in \mathbf{A}^{*}$ such that:

$$
\mathbf{c}+\mathbf{x} \stackrel{u}{\rightarrow} \mathbf{q} \stackrel{v}{\rightarrow} \mathbf{c}+\mathbf{y} \quad \text { and } \quad \mathbf{c}+\mathbf{x}^{\prime} \stackrel{u^{\prime}}{\rightarrow} \mathbf{q}^{\prime} \stackrel{v^{\prime}}{\rightarrow} \mathbf{c}+\mathbf{y}^{\prime}
$$

Let us introduce $\mathbf{z}=\mathbf{q}^{\prime}-\mathbf{q}$. By monotony:

$$
\begin{gathered}
\mathbf{c}+\mathbf{x}+\mathbf{x}^{\prime} \stackrel{u^{\prime}}{\rightarrow} \mathbf{q}^{\prime}+\mathbf{x} \\
\mathbf{q}+\mathbf{z}+\mathbf{x} \stackrel{v}{\rightarrow} \mathbf{c}+\mathbf{y}+\mathbf{z}+\mathbf{x} \\
\mathbf{c}+\mathbf{x}+\mathbf{z}+\mathbf{y} \stackrel{u}{\rightarrow} \mathbf{q}+\mathbf{z}+\mathbf{y} \\
\mathbf{q}^{\prime}+\mathbf{y} \stackrel{v^{\prime}}{\rightarrow} \mathbf{c}+\mathbf{y}+\mathbf{y}^{\prime}
\end{gathered}
$$

Since $\mathbf{q}^{\prime}+\mathbf{x}=\mathbf{q}+\mathbf{z}+\mathbf{x}$ and $\mathbf{q}+\mathbf{z}+\mathbf{y}=\mathbf{q}^{\prime}+\mathbf{y}$, we have proved that $\mathbf{c}+\mathbf{x}+\mathbf{x}^{\prime} \stackrel{u^{\prime} v}{\longrightarrow} \mathbf{c}+\mathbf{h} \stackrel{u v^{\prime}}{\longrightarrow} \mathbf{c}+\mathbf{y}+\mathbf{y}^{\prime}$ with $\mathbf{h}=\mathbf{x}+\mathbf{z}+\mathbf{y}$. Thus $\mathbf{h}$ is an intraproduction. Observe that $\mathbf{q}+\mathbf{h}=\mathbf{q}^{\prime}+\mathbf{x}+\mathbf{y} \geq \mathbf{q}^{\prime}$.

Lemma VIII.7. There exist intraproductions $\mathbf{h} \in \mathbf{H}_{\gamma}$ such that $I_{\gamma}=\{i \mid \mathbf{h}(i)=0\}$.

Proof: Let $i \notin I_{\gamma}$. There exists a sequence $\left(\mathbf{q}_{k}\right)_{k \in \mathbb{N}}$ of configurations $\mathbf{q}_{k} \in \mathbf{Q}_{\gamma}$ such that $\left(\mathbf{q}_{k}(i)\right)_{k \in \mathbb{N}}$ is strictly increasing. Since $\left(\mathbb{N}^{d}, \leq\right)$ is well-ordered there exists $k<k^{\prime}$ such that $\mathbf{q}_{k} \leq \mathbf{q}_{k^{\prime}}$. Lemma VIII.6 shows that there exists an intraproduction $\mathbf{h}_{i}$ for $\gamma$ such that $\mathbf{q}_{k^{\prime}} \leq \mathbf{q}_{k}+\mathbf{h}_{i}$. In particular $\mathbf{h}_{i}(i)>0$. As the set of intraproductions $\mathbf{H}_{\gamma}$ is periodic we deduce that $\mathbf{h}=\sum_{i \notin I} \mathbf{h}_{i}$ is an intraproduction for $\gamma$. By construction we have $\mathbf{h}(i)>0$ for every $i \notin I_{\gamma}$. Since $\mathbf{h} \in \mathbf{H}_{\gamma}$ we deduce that $\mathbf{h}(i)=0$ for every $i \in I_{\gamma}$. Therefore $I_{\gamma}=\{i \mid \mathbf{h}(i)=0\}$.

Given $s \in S_{\gamma}$ we introduce the relation $R_{\gamma, s}$ of pairs $(\mathbf{e}, \mathbf{f}) \in \mathbb{Q}_{\geq 0}^{d} \times \mathbb{Q}_{\geq 0}^{d}$ such that $\mathbf{f}-\mathbf{e} \in \mathbb{Q}_{\geq 0} \Delta(\sigma)$ where $\sigma$ is the label of a cycle on $s$ in $G_{\gamma}$. Observe that $R_{\gamma, s}$ is a reflexive definable conic relation. From Theorem VII.1 we deduce that the transitive closure $R_{\gamma}=\left(\bigcup_{s \in S_{\gamma}} R_{\gamma, s}\right)^{+}$is a reflexive definable conic relation.

Example VIII.8. Let us come back to Example VIII.2. Cycles in the graph $G_{\gamma}$ (depicted in Figure 5) are labeled by words such that the number of occurrences of a is equal to the number of occurrences of $\mathbf{b}$. We deduce that $R_{\gamma, s}$ is equal $\left\{(\mathbf{e}, \mathbf{f}) \in \mathbb{Q}_{\geq 0}^{3} \times \mathbb{Q}_{\geq 0}^{3} \mid \mathbf{f}-\mathbf{e} \in \mathbb{Q}_{\geq 0} \mathbf{y}\right\}$ whatever the state $s \in S_{\gamma}$. We derive that $R_{\gamma}$ is also equal to this relation.

Lemma VIII.9. For every $s_{1}, \ldots, s_{k} \in S_{\gamma}$ there exists $(\mathbf{x}, \mathbf{y}) \in P$ and $\mathbf{q}_{1}, \ldots, \mathbf{q}_{k} \in \mathbf{Q}_{\gamma}$ such that $s_{j}=\pi_{\gamma}\left(\mathbf{q}_{j}\right)$ for every $1 \leq j \leq k$ and such that:

$$
\mathbf{c}+\mathbf{x} \stackrel{*}{\rightarrow} \mathbf{q}_{1} \cdots \stackrel{*}{\rightarrow} \mathbf{q}_{k} \stackrel{*}{\rightarrow} \mathbf{c}+\mathbf{y}
$$

Proof: Since $s_{j} \in S_{\gamma}$ there exists $\mathbf{p}_{j} \in \mathbf{Q}_{\gamma}$ and $\left(\mathbf{x}_{j}, \mathbf{y}_{j}\right) \in P$ such that $\mathbf{c}+\mathbf{x}_{j} \stackrel{*}{\rightarrow} \mathbf{p}_{j} \stackrel{*}{\rightarrow} \mathbf{c}+\mathbf{y}_{j}$. Let us introduce $(\mathbf{x}, \mathbf{y})=\sum_{j=1}^{k}\left(\mathbf{x}_{j}, \mathbf{y}_{j}\right)$. Since $P$ is periodic, this pair is in $P$. Let us introduce $\mathbf{h}_{j}=\mathbf{y}_{1}+\cdots+\mathbf{y}_{j-1}+\mathbf{x}_{j}+\cdots+\mathbf{x}_{k}$. By monotony, since $\mathbf{c}+\mathbf{x}_{j} \stackrel{*}{\rightarrow} \mathbf{p}_{j} \stackrel{*}{\rightarrow} \mathbf{c}+\mathbf{y}_{j}$, we deduce that $\mathbf{c}+\mathbf{h}_{j} \stackrel{*}{\rightarrow} \mathbf{q}_{j} \stackrel{*}{\rightarrow} \mathbf{c}+\mathbf{h}_{j+1}$ where $\mathbf{q}_{j}=\mathbf{p}_{j}+\left(\mathbf{h}_{j}-\mathbf{x}_{j}\right)$. Since $\mathbf{h}_{j}-\mathbf{x}_{j}$ is a sum of intraproductions, we deduce that $\mathbf{h}_{j}-\mathbf{x}_{j}$ is an intraproduction. In particular $\pi_{\gamma}\left(\mathbf{q}_{j}\right)=\pi_{\gamma}\left(\mathbf{p}_{j}\right)=s_{j}$. We have proved the lemma.
Lemma VIII.10. For every $(\mathbf{e}, \mathbf{f}) \in R_{\gamma}$ there exists $(\mathbf{x}, \mathbf{y}) \in$ $P$ and $n \in \mathbb{N}_{>0}$ such that:

$$
(\mathbf{c}, \mathbf{c})+(\mathbf{x}, \mathbf{y})+n \mathbb{N}(\mathbf{e}, \mathbf{f})
$$

is a flatable subreachability relation.

Proof: Let us consider $(\mathbf{e}, \mathbf{f}) \in R_{\gamma}$. There exists a nonempty sequence $s_{1}, \ldots, s_{k}$ of states $s_{j} \in S_{\gamma}$ such that $(\mathbf{e}, \mathbf{f}) \in$ $R_{\gamma, s_{1}} \circ \cdots \circ R_{\gamma, s_{k}}$. We introduce $s_{0}, s_{k+1}$ equal to $s_{\gamma}$. Let us consider the sequence $\left(\mathbf{v}_{j}\right)_{0 \leq j \leq k}$ such that $\mathbf{v}_{0}=\mathbf{e}, \mathbf{v}_{k}=\mathbf{f}$ and such that $\left(\mathbf{v}_{j-1}, \mathbf{v}_{j}\right) \in R_{\gamma, s_{j}}$ for every $j \in\{1, \ldots, k\}$. By definition of $R_{\gamma, s_{j}}$, there exists $\lambda_{j} \in \mathbb{Q}_{\geq 0}$ and a cycle in $G_{\gamma}$ on $s_{j}$ labeled by a word $\sigma_{j}$ such that $\mathbf{v}_{j}-\mathbf{v}_{j-1}=\lambda_{j} \Delta\left(\sigma_{j}\right)$. By multiplying $(\mathbf{e}, \mathbf{f})$ by a positive natural number, we can assume without loss of generality that $\lambda_{j} \in \mathbb{N}$ for every $j \in$ $\{1, \ldots, k\}$, and $\mathbf{v}_{j} \in \mathbb{N}^{d}$ for every $j \in\{0, \ldots, k\}$. Moreover, by replacing $\sigma_{j}$ by $\sigma_{j}^{\lambda_{j}}$ we can assume that $\mathbf{v}_{j}-\mathbf{v}_{j-1}=$ $\Delta\left(\sigma_{j}\right)$.

Lemma VIII.9 shows that there exist $(\mathbf{x}, \mathbf{y}) \in P$, words $w_{0}, \ldots, w_{k} \in \mathbf{A}^{*}$, and configurations $\mathbf{q}_{1}, \ldots, \mathbf{q}_{k} \in \mathbf{Q}_{\gamma}$ such that $s_{j}=\pi_{\gamma}\left(\mathbf{q}_{j}\right)$ for every $1 \leq j \leq k$ and such that:

$$
\mathbf{c}+\mathbf{x} \stackrel{w_{0}}{\longrightarrow} \mathbf{q}_{1} \cdots \stackrel{w_{k-1}}{\longrightarrow} \mathbf{q}_{k} \stackrel{w_{k}}{\longrightarrow} \mathbf{c}+\mathbf{y}
$$

Note that $w=w_{0} \sigma_{1} w_{1} \ldots \sigma_{k} w_{k}$ is the label of a cycle on $s_{\gamma}$. Lemma VIII.7 shows that there exist intraproductions $\mathbf{h} \in \mathbf{H}_{\gamma}$ such that $I_{\gamma}=\{i \mid \mathbf{h}(i)=0\}$. Since the set of intraproductions is periodic, by multiplying $\mathbf{h}$ by a large positive natural number we can assume without loss of generality that there exists a run from $\mathbf{c}+\mathbf{h}$ labeled by $w$. As $\mathbf{h}$ is an intraproduction there exist $\left(\mathbf{x}^{\prime}, \mathbf{y}^{\prime}\right) \in P$ and $u, v \in \mathbf{A}^{*}$ such that $\mathbf{c}+\mathbf{x}^{\prime} \stackrel{u}{\rightarrow} \mathbf{c}+\mathbf{h} \stackrel{v}{\rightarrow} \mathbf{c}+\mathbf{y}^{\prime}$. By monotony, we deduce that for every $r \in \mathbb{N}$ we have:

$$
\mathbf{c}+\mathbf{x}+\mathbf{x}^{\prime}+r \mathbf{e} \stackrel{u w_{0} \sigma_{1}^{r} w_{1} \ldots \sigma_{k}^{r} w_{k} v}{\longrightarrow} \mathbf{c}+\mathbf{y}+\mathbf{y}^{\prime}+r \mathbf{f}
$$

Since $P$ is periodic we deduce that $\left(\mathbf{x}+\mathbf{x}^{\prime}, \mathbf{y}+\mathbf{y}^{\prime}\right) \in P$. We have proved the lemma with the bounded language $W=$ $u w_{0} \sigma_{1}^{*} w_{1} \ldots \sigma_{k}^{*} w_{k} v$.

\section{Lemma VIII.11. States in $S_{\gamma}$ are incomparable.}

Proof: Let us consider $s \leq s^{\prime}$ in $S_{\gamma}$. There exists $\mathbf{q}, \mathbf{q}^{\prime} \in$ $\mathbf{Q}_{\gamma}$ such that $s=\pi_{\gamma}(\mathbf{q})$ and $s^{\prime}=\pi_{\gamma}\left(\mathbf{q}^{\prime}\right)$. Lemma VIII.7 shows that there exists an intraproduction $\mathbf{h}^{\prime} \in \mathbf{H}_{\gamma}$ such that $I_{\gamma}=\left\{i \mid \mathbf{h}^{\prime}(i)=0\right\}$. By replacing $\mathbf{h}^{\prime}$ by a vector in $\mathbb{N}_{>0} \mathbf{h}^{\prime}$ we can assume without loss of generality that $\mathbf{q}(i) \leq \mathbf{q}^{\prime}(i)+$ $\mathbf{h}^{\prime}(i)$ for every $i \notin I_{\gamma}$. As $\mathbf{q}(i)=s(i) \leq s^{\prime}(i)=\mathbf{q}^{\prime}(i)=$ $\mathbf{q}^{\prime}(i)+\mathbf{h}^{\prime}(i)$ for every $i \in I_{\gamma}$ we deduce that $\mathbf{q} \leq \mathbf{q}^{\prime}+\mathbf{h}^{\prime}$. Lemma VIII.3 shows that $\mathbf{q}^{\prime}+\mathbf{h}^{\prime} \in \mathbf{Q}_{\gamma}$. Lemma VIII.6 shows that there exists an intraproduction $\mathbf{h} \in \mathbf{H}_{\gamma}$ such that $\mathbf{q}^{\prime}+\mathbf{h}^{\prime} \leq$ $\mathbf{q}+\mathbf{h}$. As $\mathbf{h} \in \mathbf{H}_{\gamma}$ we deduce that $\mathbf{h}(i)=0$ for every $i \in I_{\gamma}$. In particular $\mathbf{q}^{\prime}(i) \leq \mathbf{q}(i)$ for every $i \in I_{\gamma}$. Hence $s^{\prime} \leq s$, and we get $s=s^{\prime}$.

Lemma VIII.12. We have $\lim (P) \subseteq R_{\gamma}$.

Proof: Let $(\mathbf{e}, \mathbf{f}) \in \lim (P)$. By multiplying this pair by a positive integer, we can assume that there exists $(\mathbf{x}, \mathbf{y}) \in P$ such that $(\mathbf{x}, \mathbf{y})+\mathbb{N}(\mathbf{e}, \mathbf{f}) \subseteq \mathbf{P}$. Thus for every $n \in \mathbb{N}$ there exists a run $\rho_{n}$ labeled by a word in $\mathbf{A}^{*}$ such that $\operatorname{dir}\left(\rho_{n}\right)=$ 
$(\mathbf{c}, \mathbf{c})+(\mathbf{x}, \mathbf{y})+n(\mathbf{e}, \mathbf{f})$. Lemma VI.2 shows that there exists $n<m$ such that $\rho_{n} \unlhd \rho_{m}$. Assume that $\rho_{n}$ is the run $\mathbf{c}_{0} \ldots \mathbf{c}_{k}$ where $\mathbf{c}_{j} \in \mathbb{N}^{d}$. There exists a sequence $\mathbf{v}_{0}, \ldots, \mathbf{v}_{k+1} \in \mathbb{N}^{d}$ such that $\rho_{m}=\rho_{0}^{\prime} \ldots \rho_{k}^{\prime}$ where $\rho_{j}^{\prime}$ is a run from $\mathbf{c}_{j}+\mathbf{v}_{j}$ to $\mathbf{c}_{j}+\mathbf{v}_{j+1}$ labeled by a word $\sigma_{j}$. Observe that $s_{j}=\pi_{\gamma}\left(\mathbf{c}_{j}\right)$ is in $S_{\gamma}$. Since $s_{j} \leq \pi_{\gamma}\left(\mathbf{c}_{j}+\mathbf{v}_{j}\right)$, Lemma VIII.11 shows that $s_{j}=\pi_{\gamma}\left(\mathbf{c}_{j}+\mathbf{v}_{j}\right)$. Since $s_{j} \leq \pi_{\gamma}\left(\mathbf{c}_{j}+\mathbf{v}_{j+1}\right)$, we also deduce that $s_{j}=\pi_{\gamma}\left(\mathbf{c}_{j}+\mathbf{v}_{j+1}\right)$. Thus $\sigma_{j}$ is the label of a cycle on $s_{j}$ in $G_{\gamma}$. We deduce that $\left(\mathbf{v}_{j}, \mathbf{v}_{j+1}\right) \in R_{\gamma, s_{j}}$. Thus $\left(\mathbf{v}_{0}, \mathbf{v}_{k+1}\right) \in R_{\gamma}$. Since this pair is equal to $(\mathbf{e}, \mathbf{f})$, we are done.

We have proved Theorem VIII.1 thanks to the relation $R_{\gamma}$, denoted as $R$ in that theorem.

\section{REACHABILITY DECOMPOSITION}

In this section, we prove the following theorem. All other results are not used in the sequel.

Theorem IX.1. For every Presburger set $\mathbf{X} \subseteq \mathbb{N}^{d}$, the set $\operatorname{post}\left(\mathbf{c}_{\text {init }}, \mathbf{A}^{*}\right) \cap \mathbf{X}$ is a finite union of sets $\mathbf{b}+\mathbf{P}$ where $\mathbf{b} \in \mathbb{N}^{d}$ and $\mathbf{P} \subseteq \mathbb{N}^{d}$ is a smooth periodic set such that for every linear set $\mathbf{Y} \subseteq \mathbf{b}+\mathbf{P}$ there exists $\mathbf{p} \in \mathbf{P}$ such that $\mathbf{p}+\mathbf{Y}$ is flatable.

The proof of the previous theorem is based on the following simple lemma.

Lemma IX.2. For every relations $R_{1}, R_{2} \subseteq \mathbb{N}^{d} \times \mathbb{N}^{d}$ and for every capacity $\mathbf{c} \in \mathbb{N}^{d}$ such that $(\mathbf{c}, \mathbf{c})+R_{1}$ and $(\mathbf{c}, \mathbf{c})+R_{2}$ are flatable subreachability relations, then $(\mathbf{c}, \mathbf{c})+R_{1}+R_{2}$ is a flatable subreachability relation.

Proof: There exist bounded languages $W_{1}, W_{2} \subseteq \mathbf{A}^{*}$ such that $(\mathbf{c}, \mathbf{c})+R_{1}$ and $(\mathbf{c}, \mathbf{c})+R_{2}$ are included respectively in $\stackrel{W_{1}}{\longrightarrow}$ and $\stackrel{W_{2}}{\longrightarrow}$. By monotony, we deduce that $(\mathbf{c}, \mathbf{c})+R_{1}+$ $R_{2}$ is included in $\stackrel{W_{1} W_{2}}{\longrightarrow}$.

Since Presburger sets are finite unions of linear sets, we can assume that $\mathbf{X}$ is a linear set in the previous Theorem IX.1. Hence, we can assume that there exists a configuration $\mathbf{x} \in$ $\mathbb{N}^{d}$ and a finitely generated periodic set $\mathbf{M} \subseteq \mathbb{N}^{d}$ such that $\mathbf{X}=\mathbf{x}+\mathbf{M}$. We introduce the set $\Omega$ of runs $\rho$ from the initial configuration $\mathbf{c}_{\text {init }}$ to a configuration in $\mathbf{X}$. Lemma VI.2 shows that $\unlhd$ is a well-order over $\Omega$ and Lemma V.5 shows that $\leq_{\mathbf{M}}$ is a well-order over $\mathbf{M}$. We deduce that $\Omega$ is wellordered by the relation $\sqsubseteq$ defined by $\rho \sqsubseteq \rho^{\prime}$ if $\rho \unlhd \rho^{\prime}$ and $\operatorname{tgt}(\rho)-\mathbf{x} \leq_{\mathbf{M}} \operatorname{tgt}\left(\rho^{\prime}\right)-\mathbf{x}$. In particular $\Omega_{0}=\min _{\sqsubseteq}(\Omega)$ is a finite set. Let us observe that we have the following equality:

$$
\mathbf{X}=\bigcup_{\rho \in \Omega_{0}} \operatorname{tgt}(\rho)+\mathbf{M}_{\rho}
$$

Where $\mathbf{M}_{\rho}$ is the following periodic set:

$$
\mathbf{M}_{\rho}=\left\{\mathbf{m} \in \mathbf{M} \mid \mathbf{0} \stackrel{\mathbf{c}_{0}}{\curvearrowright} \circ \cdots \circ \stackrel{\mathbf{c}_{k}}{\curvearrowright} \mathbf{m}\right\}
$$

So, the proof of Theorem IX.1 reduces to show that $\mathbf{M}_{\rho}$ is a smooth periodic set such that for every $\mathbf{y} \in \mathbb{N}^{d}$ and for every finitely generated periodic set $\mathbf{Q} \subseteq \mathbb{N}^{d}$ such that $\mathbf{y}+\mathbf{Q} \subseteq$
$\operatorname{tgt}(\rho)+\mathbf{M}_{\rho}$, there exists $\mathbf{m} \in \mathbf{M}_{\rho}$ such that $\mathbf{y}+\mathbf{m}+\mathbf{Q}$ is flatable.

In the sequel $\rho$ is a run in $\Omega$ of the form $\rho=\mathbf{c}_{0} \ldots \mathbf{c}_{k}$. We introduce the periodic set $P$ of tuples $\left(\mathbf{x}_{0}, \ldots, \mathbf{x}_{k+1}\right) \in$ $\left(\mathbb{N}^{d}\right)^{k+2}$ such that $\mathbf{x}_{0}=\mathbf{0}, \mathbf{x}_{k+1} \in \mathbf{M}$ and $\mathbf{x}_{j} \stackrel{\mathbf{c}_{j}}{\curvearrowright} \mathbf{x}_{j+1}$ for every $j$. We consider the projection function $\pi_{j}:\left(\mathbb{N}^{d}\right)^{k+2} \rightarrow$ $\mathbb{N}^{d} \times \mathbb{N}^{d}$ defined by $\pi_{j}\left(\mathbf{x}_{0}, \ldots, \mathbf{x}_{k+1}\right)=\left(\mathbf{x}_{j}, \mathbf{x}_{j+1}\right)$. We also introduce the periodic set $P_{j}=\pi_{j}(P)$. Theorem VIII.1 shows that there exists a definable conic relation $R_{j} \subseteq \mathbb{Q}_{\geq 0}^{d} \times \mathbb{Q}_{\geq 0}^{d}$ such that $\lim \left(P_{j}\right) \subseteq R_{j}$ and such that for every $r_{j} \in R_{j}$, there exists $p_{j} \in P$ and $n_{j} \in \mathbb{N}_{>0}$ such that $\left(\mathbf{c}_{j}, \mathbf{c}_{j}\right)+\pi_{j}\left(p_{j}\right)+$ $n_{j} \mathbb{N} r_{j}$ is a flatable subreachability relation.

We introduce the following definable conic set:

$$
\mathbf{C}=\left\{\mathbf{c} \in \mathbb{Q}_{\geq 0}^{d} \mid \mathbf{0} R_{0} \circ \cdots \circ R_{k} \mathbf{c}\right\}
$$

Lemma IX.3. The periodic set $\mathbf{M}_{\rho}$ is well-limit and its limit is included in $\mathbf{C} \cap \mathbb{Q}_{\geq 0} M$.

Proof: Let us consider a sequence $\left(\mathbf{m}_{n}\right)_{n \in \mathbb{N}}$ of vectors $\mathbf{m}_{n} \in \mathbf{M}_{\rho}$. For every $n$, there exists a sequence $\left(\mathbf{x}_{0, n}, \ldots, \mathbf{x}_{k+1, n}\right)$ in $P$ such that $\mathbf{x}_{k+1, n}=\mathbf{m}_{n}$. So, there exists a run $\rho_{j, n}$ from $\mathbf{c}_{j}+\mathbf{x}_{j, n}$ to $\mathbf{c}_{j}+\mathbf{x}_{j+1, n}$ labeled by a word in $\mathbf{A}^{*}$. Lemma VI.2 shows that $\unlhd$ is a well-order over the runs and Lemma V.5 shows that $\leq_{\mathrm{M}}$ is a well-order over M. We deduce that there exists an infinite set $N \subseteq \mathbb{N}$ such that $\rho_{j, n} \unlhd \rho_{j, m}$ and $\mathbf{m}_{n} \leq_{\mathbf{M}} \mathbf{m}_{m}$ for every $n \leq m$ in $N$ and for every $0 \leq j \leq k$. Lemma VI.3 shows that for every $r \in \mathbb{N}$ there exists a run labeled by a word in $\mathbf{A}^{*}$ with a direction equals to $\operatorname{dir}\left(\rho_{j, n}\right)+r\left(\operatorname{dir}\left(\rho_{j, m}\right)-\operatorname{dir}\left(\rho_{j, n}\right)\right)$. Let us introduce $\mathbf{z}_{j, r}=\mathbf{x}_{j, n}+r\left(\mathbf{x}_{j, m}-\mathbf{x}_{j, n}\right)$ and observe that the previous direction is equal to $\left(\mathbf{c}_{j}, \mathbf{c}_{j}\right)+\left(\mathbf{z}_{j, r}, \mathbf{z}_{j+1, r}\right)$. Thus $\mathbf{z}_{j, r} \stackrel{\mathbf{c}_{j}}{\curvearrowright} \mathbf{z}_{j+1, r}$. Since $\mathbf{z}_{0, r}=\mathbf{0}$ and $\mathbf{z}_{k+1, r}=\mathbf{m}_{n}+$ $r\left(\mathbf{m}_{m}-\mathbf{m}_{n}\right) \in \mathbf{M}$ from $\mathbf{m}_{n} \leq_{\mathbf{M}} \mathbf{m}_{m}$, we deduce that $\left(\mathbf{z}_{0, r}, \ldots, \mathbf{z}_{k+1, r}\right) \in P$. Thus $\mathbf{m}_{n}+r\left(\mathbf{m}_{m}-\mathbf{m}_{n}\right) \in \mathbf{M}_{\rho}$. We deduce that $\mathbf{m}_{m}-\mathbf{m}_{n} \in \lim \left(\mathbf{M}_{\rho}\right)$. Therefore $\mathbf{M}_{\rho}$ is well-limit periodic.

Now, let us consider $\mathbf{v} \in \lim \left(\mathbf{M}_{\rho}\right)$. By multiplying this vector by a positive integer, we can assume that there exists $\mathbf{m} \in \mathbf{M}$ such that $\mathbf{m}_{n}=\mathbf{m}+n \mathbf{v}$ is in $\mathbf{M}_{\rho}$ for every $n \in \mathbb{N}$. We can then apply the previous paragraph on this sequence. Let $n<m$ in $N$. Since $\left(\mathbf{z}_{0, r}, \ldots, \mathbf{z}_{k+1, r}\right) \in P$ we deduce that $\left(\mathbf{z}_{j, r}, \mathbf{z}_{j, r+1}\right) \in P_{j}$. Thus $\left(\mathbf{x}_{j, n}, \mathbf{x}_{j+1, n}\right)+$ $\mathbb{N}\left(\left(\mathbf{x}_{j, m}, \mathbf{x}_{j+1, m}\right)-\left(\mathbf{x}_{j, n}, \mathbf{x}_{j+1, n}\right)\right)$ is included in $\mathbf{P}_{j}$ and we deduce that $\left(\mathbf{x}_{j, m}, \mathbf{x}_{j+1, m}\right)-\left(\mathbf{x}_{j, n}, \mathbf{x}_{j+1, n}\right) \in \lim \left(P_{j}\right)$. Hence $\left(\mathbf{x}_{j, m}, \mathbf{x}_{j+1, m}\right)-\left(\mathbf{x}_{j, n}, \mathbf{x}_{j+1, n}\right) \in R_{j}$. We deduce that $\left(\mathbf{x}_{0, m}-\right.$ $\left.\mathbf{x}_{0, n}, \mathbf{x}_{k+1, m}-\mathbf{x}_{k+1, n}\right) \in R_{0} \circ \cdots \circ R_{k}$. From $\mathbf{x}_{0, m}-\mathbf{x}_{0, n}=\mathbf{0}$ and $\mathbf{x}_{k+1, m}-\mathbf{x}_{k+1, n}=\mathbf{m}_{m}-\mathbf{m}_{n}=(m-n) \mathbf{v}$, we deduce that $\mathbf{v} \in \mathbf{C}$. Moreover, from $\mathbf{m}_{n} \leq_{\mathbf{M}} \mathbf{m}_{n}$ we get $(m-n) \mathbf{v} \in \mathbf{M}$. We have proved that $\mathbf{v} \in \mathbf{C} \cap \mathbb{Q}_{>0} \mathbf{M}$.

Lemma IX.4. For every $\mathbf{v} \in \mathbf{C}$, there exist relations $\tilde{R}_{0}, \ldots, \tilde{R}_{k} \subseteq \mathbb{N}^{d} \times \mathbb{N}^{d}$ such that $\left(\mathbf{c}_{j}, \mathbf{c}_{j}\right)+\tilde{R}_{j}$ is a flatable subreachability relation, $\mathbf{m} \in \mathbf{M}$, and $n \in \mathbb{N}_{>0}$ such that for every $r \in \mathbb{N}$ :

$$
\mathbf{0} \tilde{R}_{0} \circ \cdots \circ \tilde{R}_{k} \mathbf{m}+r n \mathbf{v}
$$


Proof: Let us consider $\mathbf{v} \in \mathbf{C}$. There exists a sequence $\left(\mathbf{v}_{0}, \ldots, \mathbf{v}_{k+1}\right) \in\left(\mathbb{Q}_{\geq 0}^{d}\right)^{k+1}$ such that $\mathbf{v}_{0}=\mathbf{0}, \mathbf{v}_{k+1}=\mathbf{v}$ and $\left(\mathbf{v}_{j}, \mathbf{v}_{j+1}\right) \in R_{j}$ for every $j$. There exist $n_{j} \in \mathbb{N}_{>0}, p_{j} \in P$, such that $\left(\mathbf{c}_{j}, \mathbf{c}_{j}\right)+\pi_{j}\left(p_{j}\right)+n_{j} \mathbb{N}\left(\mathbf{v}_{j}, \mathbf{v}_{j+1}\right)$ is a flatable subreachability relation. Let $n=\prod_{j=0}^{k} n_{j}$. Since $n \mathbb{N} \subseteq n_{j} \mathbb{N}$ we deduce that $\left(\mathbf{c}_{j}, \mathbf{c}_{j}\right)+\pi_{j}\left(p_{j}\right)+n \mathbb{N}\left(\mathbf{v}_{j}, \mathbf{v}_{j+1}\right)$ is a flatable subreachability relation. Let us consider $p=\sum_{j=1}^{k} p_{j}$. Note that $p-p_{j} \in P$ and in particular $\left(c_{j}, c_{j}\right)+\pi_{j}\left(p-p_{j}\right)$ is in the reachability relation. Lemma IX.2 shows that $\left(c_{j}, c_{j}\right)+\tilde{R}_{j}$ is a flatable subreachability relation where $\tilde{R}_{j}=\pi_{j}(p)+$ $n \mathbb{N}\left(\mathbf{v}_{j}, \mathbf{v}_{j+1}\right)$. Assume that $p=\left(\mathbf{x}_{0}, \ldots, \mathbf{x}_{k+1}\right)$. We have proved that for every $r \in \mathbb{N}$ we have $\mathbf{x}_{j}+n r \mathbf{v}_{j} \tilde{R}_{j} \mathbf{x}_{j+1}+$ $n r \mathbf{v}_{j+1}$. Since $p \in P$ we deduce that $\mathbf{x}_{0}=\mathbf{0}$ and $\mathbf{m}=\mathbf{x}_{k+1}$ is a vector in $\mathbf{M}$. Since $\mathbf{v}_{0}=\mathbf{0}$ and $\mathbf{v}_{k+1}=\mathbf{v}$, we have proved the lemma.

The previous Lemma IX.4 shows that $\mathbf{C} \cap \mathbb{Q}_{\geq 0} \mathbf{M}$ is included in $\lim \left(\mathbf{M}_{\rho}\right)$. Hence, with Lemma IX.3 we deduce that $\lim \left(\mathbf{M}_{\rho}\right)$ is equal to the definable conic set $\mathbf{C} \cap \mathbb{Q}_{\geq 0} \mathbf{M}$.

Lemma IX.5. For every $\mathbf{y} \in \mathbb{N}^{d}$ and for every finitely generated periodic set $\mathbf{Q} \subseteq \mathbb{N}^{d}$ such that $\mathbf{y}+\mathbf{Q} \subseteq \operatorname{tgt}(\rho)+\mathbf{M}_{\rho}$, there exists $\mathbf{m} \in \mathbf{M}_{\rho}$ such that $\mathbf{y}+\mathbf{m}+\mathbf{Q}$ is flatable.

Proof: Since $\mathbf{Q}$ is finitely generated, there exists a finite set $\mathbf{V} \subseteq \mathbf{Q}$ that generates $\mathbf{Q}$. Observe that $\mathbf{x}-\operatorname{tgt}(\rho)+\mathbb{N} \mathbf{v} \subseteq$ $\mathbf{M}_{\rho}$ for every $\mathbf{v} \in \mathbf{V}$. Thus $\mathbf{v} \in \lim \left(\mathbf{M}_{\rho}\right)$. As $\lim \left(\mathbf{M}_{\rho}\right) \subseteq \mathbf{C} \cap$ $\mathbb{Q}_{\geq 0} \mathbf{M}$, we deduce that there exist relations $\tilde{R}_{0, \mathbf{v}}, \ldots, \tilde{R}_{k, \mathbf{v}} \subseteq$ $\mathbb{N}^{d} \times \mathbb{N}^{d}$ such that $\left(\mathbf{c}_{j}, \mathbf{c}_{j}\right)+\tilde{R}_{j, \mathbf{v}}$ is a flatable subreachability relation, $\mathbf{m}_{\mathbf{v}} \in \mathbf{M}$, and $n_{\mathbf{v}} \in \mathbb{N}_{>0}$ such that for every $r \in \mathbb{N}$ :

$$
\mathbf{0} \tilde{R}_{0, \mathbf{v}} \circ \cdots \circ \tilde{R}_{k, \mathbf{v}} \mathbf{m}_{\mathbf{v}}+r n_{\mathbf{v}} \mathbf{v}
$$

Let us consider $n=\prod_{\mathbf{v} \in \mathbf{V}} n_{\mathbf{v}}, \mathbf{m}=\sum_{\mathbf{v} \in \mathbf{V}} \mathbf{m}_{\mathbf{v}}$ and $\tilde{R}_{j}=$ $\sum_{\mathbf{v} \in \mathbf{V}} \tilde{R}_{j, \mathbf{v}}$. Lemma IX.2 shows $\left(\mathbf{c}_{j}, \mathbf{c}_{j}\right)+\tilde{R}_{j}$ is a flatable subreachability relation. Moreover, since $\mathbf{Q}$ is generated by $\mathbf{V}$ we deduce that for every $\mathbf{q} \in \mathbf{Q}$ we have:

$$
\text { o } \tilde{R}_{0} \circ \cdots \circ \tilde{R}_{k} \mathbf{m}+n \mathbf{q}
$$

Now, let us consider the set $\mathbf{Z}=\sum_{\mathbf{v} \in \mathbf{V}}\{0, \ldots, n-1\} \mathbf{v}$. Observe that $\mathbf{Z}$ is finite and since $\mathbf{Z} \subseteq \mathbf{M}_{\rho}$, we deduce that for every $\mathbf{z} \in \mathbf{M}_{\rho}$, there exists $p_{\mathbf{z}}=\left(\mathbf{x}_{0, \mathbf{z}}, \ldots, \mathbf{x}_{k+1, \mathbf{z}}\right) \in P$ such that $\mathbf{x}_{k+1, \mathbf{z}}=\mathbf{z}$. Let us consider the relation $\tilde{R}_{j}^{\prime}=\bigcup_{\mathbf{z} \in \mathbf{Z}}\left(\tilde{R}_{j}+\right.$ $\left.\pi_{j}\left(p_{\mathbf{z}}\right)\right)$. Lemma IX.2 shows that $\left(\mathbf{c}_{j}, \mathbf{c}_{j}\right)+\tilde{R}_{j}^{\prime}$ is flatable. Since $\mathbf{Q}=\mathbf{Z}+n \mathbf{Q}$ we deduce that for every $\mathbf{q} \in \mathbf{Q}$ we have:

$$
\mathbf{0} \tilde{R}_{0}^{\prime} \circ \cdots \circ \tilde{R}_{k}^{\prime} \mathbf{m}+\mathbf{q}
$$

Finally, since $\mathbf{y}-\operatorname{tgt}(\rho) \in \mathbf{M}_{\rho}$ we deduce that there exists $p=$ $\left(\mathbf{x}_{0}, \ldots, \mathbf{x}_{k+1}\right)$ in $P$ such that $\mathbf{x}_{k+1}=\mathbf{y}-\operatorname{tgt}(\rho)$. Lemma IX.2 shows that $\tilde{R}_{j}^{\prime \prime}=\tilde{R}_{j}^{\prime}+\pi_{j}(p)$ is such that $\left(\mathbf{c}_{j}, \mathbf{c}_{j}\right)+\tilde{R}_{j}^{\prime \prime}$ is flatable. Hence, this relation is included in $\stackrel{W_{j}}{\longrightarrow}$ where $W_{j} \subseteq$ $\mathbf{A}^{*}$ is a bounded language.

Let us introduce the actions $\mathbf{a}_{j}=\mathbf{c}_{j}-\mathbf{c}_{j-1}$ and the bounded language $W=W_{0} \mathbf{a}_{1} W_{1} \ldots \mathbf{a}_{k} W_{k}$. We have proved that $\operatorname{post}\left(\mathbf{c}_{\text {init }}, W\right)$ contains $\mathbf{y}+\mathbf{m}+\mathbf{Q}$. Thus this set is flatable.

We have proved Theorem IX.1.

\section{Equivalent Presburger Sets}

In this section, we first extend the notion of dimension introduced in [18] for sets included in $\mathbb{Z}^{d}$ to sets included in $\mathbb{Q}^{d}$. This definition provides a simple way for defining an equivalence relation over the subsets of $\mathbb{Q}^{d}$. Finally, we provide a characterization of sets equivalent to Presburger sets and that can be decomposed as finite unions of sets of the form $\mathbf{b}+\mathbf{P}$ where $\mathbf{b} \in \mathbb{Z}^{d}$ and $\mathbf{P} \subseteq \mathbb{Z}^{d}$ is a smooth periodic set.

The dimension of a set $\mathbf{X} \subseteq \mathbb{Q}^{d}$ is the minimal integer $r \in\{-1, \ldots, d\}$ such that $\mathbf{X} \subseteq \bigcup_{j=1}^{k}\left(\mathbf{B}_{j}+\mathbf{V}_{j}\right)$ where $\mathbf{B}_{j}$ is a bounded subset of $\mathbb{Q}^{d}$ and $\mathbf{V}_{j} \subseteq \mathbb{Q}^{d}$ is a vector space satisfying $\operatorname{rank}\left(\mathbf{V}_{j}\right) \leq r$ for every $j$. We denote by $\operatorname{dim}(\mathbf{X})$ the dimension of $\mathbf{X}$. Observe that $\operatorname{dim}(\mathbf{v}+\mathbf{X})=\operatorname{dim}(\mathbf{X})$ for every $\mathbf{X} \subseteq \mathbb{Q}^{d}$ and for every $\mathbf{v} \in \mathbb{Q}^{d}$. Observe that $\operatorname{dim}(\mathbf{X})=$ -1 if and only if $\mathbf{X}$ is empty. Note that $\operatorname{dim}(\mathbf{X} \cup \mathbf{Y})=$ $\max \{\operatorname{dim}(\mathbf{X}), \operatorname{dim}(\mathbf{Y})\}$ for every subsets $\mathbf{X}, \mathbf{Y} \subseteq \mathbb{Q}^{d}$.

Example X.1. $\operatorname{dim}(\mathbb{N})=1, \operatorname{dim}(\mathbb{Q})=1, \operatorname{dim}(\mathbb{N}(1,0)+$ $\mathbb{N}(1,1))=2, \operatorname{dim}(\mathbb{N}(1,0) \cup \mathbb{N}(1,1))=1$.

The dimension of a periodic set is obtained as follows.

Lemma X.2. We have $\operatorname{dim}(\mathbf{P})=\operatorname{rank}(\mathbf{V})$ for every periodic set $\mathbf{P}$ where $\mathbf{V}$ is the vector space generated by $\mathbf{P}$.

Given a natural number $r \in\{0, \ldots, d\}$, we introduce the equivalence relation $\equiv_{r}$ over the subsets of $\mathbb{Q}^{d}$ by $\mathbf{X} \equiv_{r} \mathbf{Y}$ if $\operatorname{dim}(\mathbf{X} \Delta \mathbf{Y})<r$. Note that $\equiv_{r}$ is distributive over $\cup$ and $\cap$. In the appendix, the following Theorem X.3 is proved.

Theorem X.3. Let $\mathbf{X}=\bigcup_{j=1}^{k}\left(\mathbf{b}_{j}+\mathbf{P}_{j}\right)$ where $\mathbf{b}_{j} \in \mathbb{Z}^{d}$ and $\mathbf{P}_{j} \subseteq \mathbb{Z}^{d}$ is a smooth periodic set. We assume that $\mathbf{X}$ is non empty and we introduce $r=\operatorname{dim}(\mathbf{X})$. If $\mathbf{X}$ is equivalent for $\equiv_{r}$ to a Presburger set then there exists a sequence $\left(\mathbf{Y}_{j}\right)_{1 \leq j \leq k}$ of linear sets $\mathbf{Y}_{j} \subseteq \mathbf{b}_{j}+\mathbf{P}_{j}$ such that $\mathbf{X} \equiv_{r} \bigcup_{j=1}^{k} \mathbf{p}_{j}+\mathbf{Y}_{j}$ for every sequence $\left(\mathbf{p}_{j}\right)_{1 \leq j \leq k}$ of vectors $\mathbf{p}_{j} \in \mathbf{P}_{j}$.

\section{Presburger Reachability Sets}

In this section we prove that Presburger subreachability sets are flatable. As a direct consequence, we deduce that Presburger VAS are flatable.

Lemma XI.1. Presburger subreachability sets are flatable.

Proof: We prove by induction over $r \in\{-1, \ldots, d\}$ that Presburger subreachability sets $\mathbf{X}$ with $\operatorname{dim}(\mathbf{X}) \leq r$ are flatable. Note that if $\operatorname{dim}(\mathbf{X})=-1$ then $\mathbf{X}$ is empty and the proof is immediate. Let us assume that the lemma is proved in dimension $r \in\{-1, \ldots, d\}$ and let us consider a Presburger subreachability set $\mathbf{X} \subseteq \operatorname{post}\left(\mathbf{c}_{\text {init }}, \mathbf{A}^{*}\right)$ such that $\operatorname{dim}(\mathbf{X})=$ $r+1$. In particular $\mathbf{X}$ is non empty. Theorem IX.1 shows $\operatorname{post}\left(\mathbf{c}_{\text {init }}, \mathbf{A}^{*}\right) \cap \mathbf{X}$ is a finite union of sets $\bigcup_{j=1}^{k}\left(\mathbf{b}_{j}+\mathbf{P}_{j}\right)$ where $\mathbf{b}_{j} \in \mathbb{N}^{d}$ and $\mathbf{P}_{j} \subseteq \mathbb{N}^{d}$ is a smooth periodic set such that for every linear set $\mathbf{Y}_{j} \subseteq \mathbf{b}_{j}+\mathbf{P}_{j}$ there exists $\mathbf{p}_{j} \in \mathbf{P}_{j}$ such that $\mathbf{p}_{j}+\mathbf{Y}_{j}$ is flatable.

Since post $\left(\mathbf{c}_{\text {init }}, \mathbf{A}^{*}\right) \cap \mathbf{X}$ is equal to $\mathbf{X}$ which is a Presburger set, Theorem X.3 shows that there exists a sequence 
$\left(\mathbf{Y}_{j}\right)_{1 \leq j \leq k}$ of linear sets $\mathbf{Y}_{j} \subseteq \mathbf{b}_{j}+\mathbf{P}_{j}$ such that $\mathbf{X} \equiv_{r}$ $\bigcup_{j=1}^{k} \mathbf{p}_{j}+\mathbf{Y}_{j}$ for every sequence $\left(\mathbf{p}_{j}\right)_{1 \leq j \leq k}$ of vectors $\mathbf{p}_{j} \in \mathbf{P}_{j}$.

Let us consider a sequence $\left(\mathbf{p}_{j}\right)_{1 \leq j \leq k}$ of vectors $\mathbf{p}_{j} \in \mathbf{P}_{j}$ such that $\mathbf{p}_{j}+\mathbf{Y}_{j}$ is flatable. We deduce that $\mathbf{Y}=\bigcup_{j=1}^{k} \mathbf{p}_{j}+$ $\mathbf{Y}_{j}$ is flatable. Since $\mathbf{X} \equiv{ }_{r} \mathbf{Y}$ we deduce that $\operatorname{dim}(\mathbf{X} \backslash \mathbf{Y})<r$. Since $\mathbf{X} \backslash \mathbf{Y}$ is a Presburger subreachability set, by induction, this set is flatable. From $\mathbf{X} \subseteq(\mathbf{X} \backslash \mathbf{Y}) \cup \mathbf{Y}$, we deduce that $\mathbf{X}$ is flatable. We have proved the rank $r+1$.

Theorem XI.2. The class of flatable VAS coincides with the class of Presburger VAS.

Proof: Assume first that the VAS is Presburger. Then $\mathbf{X}=\operatorname{post}\left(\mathbf{c}_{\text {init }}, \mathbf{A}^{*}\right)$ is a Presburger set. The previous lemma shows that $\mathbf{X}$ is flatable. Hence the VAS is flatable. Conversely, if the VAS is flatable, Theorem III.4 shows that the VAS is Presburger.

Corollary XI.3. Presburger subreachability relations are flatable.

Proof: Let $\mathbf{A} \subseteq \mathbb{Z}^{d}$ be a finite set of actions. We consider the $\operatorname{VAS}\left((\mathbf{0}, \mathbf{0}), A^{\prime}\right)$ in dimension $2 d$ where $A^{\prime}$ is the set $\{\mathbf{0}\} \times$ $\mathbf{A}$ and the vectors $\left(\mathbf{u}_{i}, \mathbf{u}_{i}\right)$ where $\mathbf{u}_{i} \in \mathbb{Z}^{d}$ satisfies $\mathbf{u}_{i}(j)=0$ if $j \neq i$ and $\mathbf{u}_{i}(i)=1$. Observe that the reachability set of this VAS is $\stackrel{\mathbf{A}^{*}}{\longrightarrow}$. Hence, if a subreachability relation $R$ of $\stackrel{\mathbf{A}^{*}}{\longrightarrow}$ is Presburger, we deduce that there exists a bounded language $W^{\prime} \subseteq\left(A^{\prime}\right)^{*}$ such that $R \subseteq \operatorname{post}\left((\mathbf{0}, \mathbf{0}), W^{\prime}\right)$. Let us consider the word morphism $\phi:\left(A^{\prime}\right)^{*} \rightarrow A^{*}$ defined by $\phi(\mathbf{0}, \mathbf{a})=\mathbf{a}$ and $\phi\left(\mathbf{u}_{i}, \mathbf{u}_{i}\right)=\varepsilon$. Observe that $W=\phi\left(W^{\prime}\right)$ is a bounded language and post $\left((\mathbf{0}, \mathbf{0}), W^{\prime}\right)$ is included in $\stackrel{W}{\longrightarrow}$. We deduce that $R$ is flatable.

Example XI.4. Let $\mathbf{A}=\{\mathbf{a}, \mathbf{b}, \mathbf{c}\}$ with $\mathbf{a}=(1,1,1), \mathbf{b}=$ $(-1,2,1)$ and $\mathbf{c}=(2,-1,1)$. The reachability relation $\stackrel{*}{\rightarrow}$ is equal to $\stackrel{W}{\longrightarrow}$ with $W=\mathbf{a}^{*} \mathbf{b}^{*} \mathbf{c}^{*} \mathbf{b}^{*}(\mathbf{b c})^{*} \mathbf{b}^{*} \mathbf{c}^{*}$.

\section{CONCLUSION}

This paper proved that acceleration techniques are complete for computing Presburger formulas denoting reachability sets of Presburger VAS. Since there exist VAS with finite reachability sets of Ackermann cardinals [20], acceleration-based algorithms have an Ackermann lower bound of complexity. Note that deciding reachability problems for Presburger VAS in Ackermannian complexity is an open problem. Moreover, an Ackermannian worst case complexity does not prevent algorithms like the Karp and Miller one [21] to decide some reachability problems (so-called coverability problems) efficiently in practice.

In the future, we are interested in improving acceleration techniques to avoid the Presburger definability condition of the reachability sets. As a first step, we are interested in characterizing vector addition systems with reachability sets not definable in the Presburger arithmetic. These vector addition systems are interesting since we know that there exist inductive invariants definable in the Presburger arithmetic obtained by over-approximating reachability sets. The main objective is an algorithm for deciding the general reachability problem for vector addition systems based on accelerations and on-demand over-approximations that works well in practice.

\section{ACKNOWLEDGMENT}

This work was supported by the ANR project REACHARD (ANR-11-BS02-001)

\section{REFERENCES}

[1] J. Esparza and M. Nielsen, "Decidability issues for petri nets - a survey," Bulletin of the European Association for Theoretical Computer Science, vol. 52, pp. 245-262, 1994.

[2] G. S. Sacerdote and R. L. Tenney, "The decidability of the reachability problem for vector addition systems (preliminary version)," in Proc. of STOC'77. ACM, 1977, pp. 61-76.

[3] E. W. Mayr, "An algorithm for the general petri net reachability problem," in Proc. of STOC'81. ACM, 1981, pp. 238-246.

[4] S. R. Kosaraju, "Decidability of reachability in vector addition systems (preliminary version)," in Proc. of STOC'82. ACM, 1982, pp. 267-281.

[5] J. L. Lambert, "A structure to decide reachability in petri nets," Theoretical Computer Science, vol. 99, no. 1, pp. 79-104, 1992.

[6] D. Figueira, S. Figueira, S. Schmitz, and P. Schnoebelen, "Ackermannian and primitive-recursive bounds with dickson's lemma," in Proc. of LICS 2011. IEEE Computer Society, 2011, pp. 269-278.

[7] J. Leroux, "The general vector addition system reachability problem by Presburger inductive invariants," in Proc. of LICS'09, 2009, pp. 4-13.

[8] S. Ginsburg and E. H. Spanier, "Semigroups, Presburger formulas and languages," Pacific Journal of Mathematics, vol. 16, no. 2, pp. 285-296, 1966.

[9] J. E. Hopcroft and J.-J. Pansiot, "On the reachability problem for 5dimensional vector addition systems," Theoritical Computer Science, vol. 8, pp. 135-159, 1979.

[10] D. Hauschildt, "Semilinearity of the reachability set is decidable for Petri nets." Ph.D. dissertation, University of Hamburg, 1990.

[11] L. Fribourg, "Petri nets, flat languages and linear arithmetic," in Proc. of WFLP'2000, M. Alpuente, Ed., 2000, pp. 344-365.

[12] J. Leroux and G. Sutre, "Flat counter automata almost everywhere!" in Proc. of ATVA'05, ser. LNCS, vol. 3707. Springer, 2005, pp. 489-503.

[13] S. Ginsburg and E. Spanier, "Bounded regular sets," Proceedings of the American Mathematical Society, vol. 17, no. 5, pp. 1043-1049, 1966.

[14] P. Jančar, "Decidability of a temporal logic problem for petri nets," Theoretical Computer Science, vol. 74, no. 1, pp. 71-93, 1990.

[15] J. Leroux, "Presburger vector addition systems," HAL, Tech. Rep., 2013. [Online]. Available: http://hal.archives-ouvertes.fr/hal-00780462/

[16] A. Finkel and J. Leroux, "How to compose presburger-accelerations: Applications to broadcast protocols," in Proc. of FSTTCS'02, ser. LNCS, vol. 2556. Springer, 2002, pp. 145-156.

[17] A. Schrijver, Theory of Linear and Integer Programming. New York: John Wiley and Sons, 1987.

[18] J. Leroux, "Vector addition system reachability problem: a short selfcontained proof," in Proc. of POPL'11, ser. POPL '11. ACM, 2011, pp. 307-316.

[19] - "Vector addition systems reachability problem (a simpler solution)," in The Alan Turing Centenary Conference, Turing-100, Manchester UK June 22-25, 2012, Proceedings, ser. EPiC Series, A. Voronkov, Ed., vol. 10. EasyChair, 2012, pp. 214-228.

[20] E. W. Mayr and A. R. Meyer, "The complexity of the finite containment problem for petri nets," J. ACM, vol. 28, no. 3, pp. 561-576, 1981.

[21] R. M. Karp and R. E. Miller, "Parallel program schemata," Journal of Computer and System Sciences, vol. 3, no. 2, pp. 147 - 195, 1969. 


\section{APPENDIX A}

\section{PROOF OF LEMMA V.5}

Lemma V.5. Let $\mathbf{P}$ be a discrete periodic set. The following conditions are equivalent:

- $\mathbf{P}$ is finitely generated as a periodic set.

- $\left(\mathbf{P}, \leq_{\mathbf{P}}\right)$ is well-preordered.

- $\mathbb{Q}_{\geq 0} \mathbf{P}$ is finitely generated as a conic set.

Proof: Let us consider a discrete periodic set $\mathbf{P} \subseteq \mathbb{Q}^{d}$. By replacing $\mathbf{P}$ by $n \mathbf{P}$ for some $n \in \mathbb{N}_{>0}$ we can assume in the sequel that $\mathbf{P} \subseteq \mathbb{Z}^{d}$.

Assume first that $\left(\mathbf{P}, \leq_{\mathbf{P}}\right)$ is well-preordered and let us prove that $\mathbf{P}$ is finitely generated as a periodic set. We introduce the relation $\sqsubseteq$ over $\mathbf{P}$ defined by $\mathbf{p} \sqsubseteq \mathbf{q}$ if $\mathbf{p} \leq_{\mathbf{P}} \mathbf{q}$ and if $|\mathbf{p}(i)| \leq|\mathbf{q}(i)|$ and $\mathbf{p}(i) \mathbf{q}(i) \geq 0$ for every $i \in\{1, \ldots, d\}$. Since $\leq$ is a well-order over $\mathbb{N}^{d}$ we deduce that $\sqsubseteq$ is a well-order over $\mathbf{P}$. The set $\mathbf{M}$ of minimal elements of $\mathbf{P} \backslash\{\mathbf{0}\}$ for this order is finite. We denote by $\mathbf{Q}$ be the periodic set generated by $\mathbf{M}$. Observe that $\mathbf{Q} \subseteq \mathbf{P}$. Assume by contradiction that $\mathbf{P} \backslash \mathbf{Q}$ is non empty and let us consider an element $\mathbf{p}$ in this set minimal for $\sqsubseteq$. Since $\mathbf{0} \in \mathbf{Q}$ we deduce that $\mathbf{p} \in \mathbf{P} \backslash\{\mathbf{0}\}$. Thus there exists $\mathbf{m} \in \mathbf{M}$ such that $\mathbf{m} \sqsubseteq \mathbf{p}$. Let $\mathbf{q}=\mathbf{p}-\mathbf{m}$. Since $\mathbf{m} \leq_{\mathbf{p}} \mathbf{p}$ we get $\mathbf{q} \in \mathbf{P}$. Moreover, $\mathbf{q} \sqsubseteq \mathbf{p}$. Thus, if $\mathbf{q} \notin \mathbf{Q}$, by minimality of $\mathbf{p}$ we get $\mathbf{q}=\mathbf{p}$ and $\mathbf{m}=\mathbf{0}$ which is impossible since $\mathbf{M} \subseteq \mathbf{P} \backslash\{\mathbf{0}\}$. Thus $\mathbf{q} \in \mathbf{Q}$. From $\mathbf{p}=\mathbf{q}+\mathbf{m}$ we get $\mathbf{p} \in \mathbf{Q}$ and we get a contradiction. Thus $\mathbf{P} \backslash \mathbf{Q}$ is empty and we get $\mathbf{P}=\mathbf{Q}$. In particular $\mathbf{P}$ is finitely generated as a periodic set.

Now, assume that $\mathbf{P}$ is finitely generated as a periodic set and let us prove that $\mathbf{C}=\mathbb{Q}_{>0} \mathbf{P}$ is finitely generated as a conic set. We have $\mathbf{P}=\mathbb{N} \mathbf{p}_{1}+\cdots+\mathbb{N} \mathbf{p}_{k}$ for some vectors $\mathbf{p}_{1}, \ldots, \mathbf{p}_{k} \in \mathbf{P}$. In particular $\mathbf{C}=\mathbb{Q}_{\geq 0} \mathbf{p}_{1}+\cdots+\mathbb{Q}_{\geq 0} \mathbf{p}_{k}$ and we deduce that $\mathbf{C}$ is finitely generated as a conic set.

Finally assume that $\mathbf{C}=\mathbb{Q}>0 \mathbf{P}$ is finitely generated as a conic set and let us prove that $\left(\mathbf{P}, \leq_{\mathbf{P}}\right)$ is well-preordered. There exists some vectors $\mathbf{q}_{1}, \ldots, \mathbf{q}_{k} \in \mathbf{C}$ such that $\mathbf{C}=$ $\mathbb{Q}_{\geq 0} \mathbf{q}_{1}+\cdots+\mathbb{Q}_{\geq 0} \mathbf{q}_{k}$. Since $\mathbf{C}=\mathbb{Q}_{\geq 0} \mathbf{P}$ by multiplying vectors $\mathbf{q}_{j}$ by a positive natural number, we can assume that $\mathbf{q}_{j} \in \mathbf{P}$. We denote by $\mathbf{Q}$ the periodic set generated by $\mathbf{q}_{1}, \ldots, \mathbf{q}_{k}$. Let us introduce the following set:

$$
\mathbf{B}=(\mathbf{P}-\mathbf{P}) \cap\left([0,1] \mathbf{p}_{1}+\cdots+[0,1] \mathbf{p}_{k}\right)
$$

Note that $\mathbf{B}$ is bounded and vectors in this set are in $\mathbb{Z}^{d}$. Thus $\mathbf{B}$ is finite. Let us prove that $\mathbf{P} \subseteq \mathbf{B}+\mathbf{Q}$. Note that for every $\mathbf{p} \in \mathbf{P}$ from $\mathbf{P} \subseteq \mathbf{C}$, we deduce that there exists $\lambda_{1}, \ldots, \lambda_{k} \in \mathbb{Q}_{\geq 0}$ such that $\mathbf{p}=\sum_{j=1}^{k} \lambda_{j} \mathbf{q}_{j}$. There exists $\mu_{j} \in[0,1]$ and $n_{j} \in \mathbb{N}$ such that $\lambda_{j}=\mu_{j}+n_{j}$. In particular $\mathbf{p}=\mathbf{b}+\mathbf{q}$ where $\mathbf{b}=\sum_{j=1}^{k} \mu_{j} \mathbf{q}_{j}$ and $\mathbf{q}=\sum_{j=1}^{k} n_{j} \mathbf{q}_{j}$. Note that $\mathbf{q} \in \mathbf{Q}$ and $\mathbf{b} \in \mathbf{B}$.

Now, let us consider an infinite sequence $\left(\mathbf{p}_{n}\right)_{n \in \mathbb{N}}$ of vectors in $\mathbf{P}$. For every $n \in \mathbb{N}$ there exists $\mathbf{b}_{n} \in \mathbf{B}$ and $\mathbf{q}_{n} \in \mathbf{Q}$ such that $\mathbf{p}_{n}=\mathbf{b}_{n}+\mathbf{q}_{n}$. Since $(\mathbf{B},=)$ and $\left(\mathbf{Q}, \leq_{\mathbf{Q}}\right)$ are two well-ordered sets, Dickson's Lemma show that there exists an infinite set $N \subseteq \mathbb{N}$ such that $\mathbf{b}_{n}=\mathbf{b}_{m}$ and $\mathbf{q}_{n} \leq_{\mathbf{Q}} \mathbf{q}_{m}$ for every $n \leq m$ in $N$. Thus $\mathbf{p}_{n} \leq_{\mathbf{P}} \mathbf{p}_{m}$ for every $n \leq m$ in $N$. We have proved that $\left(\mathbf{P}, \leq_{\mathbf{P}}\right)$ is well-preordered. 


\section{APPENDIX B}

\section{PROOF OF THEOREM VII.1}

In this section we prove the following theorem.

Theorem VII.1. Transitive closures of finite unions of reflexive definable conic relations over $\mathbb{Q}_{\geq 0}^{d}$ are reflexive definable conic relations.

The following lemma shows that the transitive closure of $R_{1} \cup \ldots \cup R_{k}$ where $R_{j}$ is a definable conic relation for every $j$ is equal to the transitive closure of the reflexive definable conic relation $R=R_{1} \circ \cdots \circ R_{k}$. That means Theorem VII.1 reduces to show that the class of reflexive definable conic relation over $\mathbb{Q}_{>0}^{d}$ is stable by transitive closure.

Lemma B.1. For every reflexive conic relations $R_{1}, \ldots, R_{k}$ over $\mathbb{Q}_{\geq 0}^{d}$, we have:

$$
R_{1} \cup \ldots \cup R_{k} \subseteq R_{1}+\cdots+R_{k} \subseteq R_{1} \circ \cdots \circ R_{k}
$$

Proof: Since $(\mathbf{0}, \mathbf{0}) \in R_{j}$ for every $j$ we deduce that $R_{1} \cup \ldots \cup R_{k} \subseteq R_{1}+\cdots+R_{k}$. Let us consider a sequence $\left(\mathbf{x}_{j}, \mathbf{y}_{j}\right)_{1 \leq j \leq k}$ of couples $\left(\mathbf{x}_{j}, \mathbf{y}_{j}\right) \in R_{j}$. We introduce $\mathbf{z}_{j}=$ $\mathbf{y}_{1}+\cdots+\mathbf{y}_{j}+\mathbf{x}_{j+1}+\cdots+\mathbf{x}_{k}$. Let $j \in\{1, \ldots k\}$. Since $\mathbf{z}_{j-1}-\mathbf{x}_{j} \in \mathbb{Q}_{\geq 0}^{d}$ and $R_{j}$ is a reflexive relation we get $\left(\mathbf{z}_{j-1}-\right.$ $\left.\mathbf{x}_{j}, \mathbf{z}_{j-1}-\mathbf{x}_{j}\right) \in R_{j}$. Moreover, as $\left(\mathbf{x}_{j}, \mathbf{y}_{j}\right) \in R_{j}$ and $R_{j}$ is conic we get $\left(\mathbf{z}_{j-1}-\mathbf{x}_{j}, \mathbf{z}_{j-1}-\mathbf{x}_{j}\right)+\left(\mathbf{x}_{j}, \mathbf{y}_{j}\right) \in R_{j}$. This couple is equal to $\left(\mathbf{z}_{j-1}, \mathbf{z}_{j}\right)$. We have proved that $\left(\mathbf{z}_{0}, \mathbf{z}_{k}\right) \in$ $R_{1} \circ \cdots \circ R_{k}$. Now just observe that $\left(\mathbf{z}_{0}, \mathbf{z}_{k}\right)=\sum_{j=1}^{k}\left(\mathbf{x}_{j}, \mathbf{y}_{j}\right)$.

Transitive closures of reflexive conic relations can be characterized as follows. We introduce the function $\nabla: \mathbb{Q}_{\geq 0}^{d} \times$ $\mathbb{Q}_{\geq 0}^{d} \rightarrow \mathbb{Q}^{d}$ defined by $\nabla(\mathbf{x}, \mathbf{y})=\mathbf{y}-\mathbf{x}$. Given a set $I \subseteq\{1, \ldots, d\}$ we introduce $\mathbb{Q}_{I}^{d}=\left\{\mathbf{x} \in \mathbb{Q}_{>0}^{d} \mid \mathbf{x}(i)>\right.$ $0 \Longleftrightarrow i \in I\}$ and the function $\nabla_{I}: \mathbb{Q}_{>0}^{d} \times \mathbb{Q}_{>0}^{d} \rightarrow \mathbb{Q}^{d}$ partially defined over $\mathbb{Q}_{I}^{d} \times \mathbb{Q}_{I}^{d}$ by $\nabla_{I}(r)=\nabla(r)$ for every $r \in \mathbb{Q}_{I}^{d} \times \mathbb{Q}_{I}^{d}$.

Lemma B.2. We have $\nabla_{I}^{-1}\left(\nabla_{I}(R)\right) \subseteq R^{+}$for every $I \subseteq$ $\{1, \ldots, d\}$ and for every reflexive conic relation $R$ over $\mathbb{Q}_{\geq 0}^{d}$.

Proof: Let $(\mathbf{x}, \mathbf{y}) \in \nabla_{I}^{-1}\left(\nabla_{I}(R)\right)$. Then $\mathbf{x}, \mathbf{y} \in \mathbb{Q}_{I}^{d}$. We introduce the vector $\mathbf{z} \in \mathbb{Q}_{I}^{d}$ defined by $\mathbf{z}(i)=$ $\min \{\mathbf{x}(i), \mathbf{y}(i)\}$. We also introduce $\mathbf{v}=\mathbf{y}-\mathbf{x}$. Since $\mathbf{v} \in \nabla_{I}(R)$, there exists $(\mathbf{a}, \mathbf{b}) \in\left(\mathbb{Q}_{I}^{d} \times \mathbb{Q}_{I}^{d}\right) \cap R$ such that $\mathbf{v}=\mathbf{b}-\mathbf{a}$. Since $\mathbf{z}, \mathbf{a} \in \mathbb{Q}_{I}^{d}$ there exists $n \in \mathbb{N}_{>0}$ such that $\frac{1}{n} \mathbf{a} \leq \mathbf{z}$. Hence there exists $\mathbf{e} \in \mathbb{Q}_{>0}^{d}$ such that $\mathbf{z}=\mathbf{e}+\frac{1}{n} \mathbf{a}$. As $R$ is reflexive we get $(\mathbf{e}, \mathbf{e}) \in R$ and since $R$ is conic we have $(\mathbf{e}, \mathbf{e})+\frac{1}{n}(\mathbf{a}, \mathbf{b}) \in R$. This couple is equal to $\left(\mathbf{z}, \mathbf{z}+\frac{1}{n} \mathbf{v}\right)$. Let $k \in\{0, \ldots, n\}$ and let us prove that $\mathbf{e}_{k}=\mathbf{x}+\frac{k}{n} \mathbf{v}-\mathbf{z}$ is in $\mathbb{Q}_{\geq 0}^{d}$. Let $i \in\{1, \ldots, d\}$. If $\mathbf{v}(i) \geq 0$ then $\mathbf{e}_{k}(i) \geq \mathbf{x}(i)-\mathbf{z}(i) \geq 0$. If $\mathbf{v}(i) \leq 0$ since $\mathbf{e}_{k}=\mathbf{y}-\frac{n-k}{n} \mathbf{v}-\mathbf{z}$ we deduce that $\mathbf{e}_{k}(i)=\mathbf{y}(i)-\frac{n-k}{n} \mathbf{v}(i)-\mathbf{z}(i) \geq \mathbf{y}(i)-\mathbf{z}(i) \geq 0$. Thus $\mathbf{e}_{k} \in \mathbb{Q}_{\geq 0}^{d}$. Since $R$ is reflexive we get $\left(\mathbf{e}_{k}, \mathbf{e}_{k}\right) \in R$. As $R$ is conic we deduce that $\left(\mathbf{e}_{k}, \mathbf{e}_{k}\right)+\left(\mathbf{z}, \mathbf{z}+\frac{1}{n} \mathbf{v}\right)$ is in $R$. Since this couple is equal to $\left(\mathbf{x}+\frac{k}{n} \mathbf{v}, \mathbf{x}+\frac{k+1}{n} \mathbf{v}\right)$ we have proved that $(\mathbf{x}, \mathbf{y}) \in R^{n}$.
Lemma B.3. Let $R$ be reflexive conic relation over $\mathbb{Q}^{d}$, let $\mathbf{v}_{0}, \ldots, \mathbf{v}_{k} \in \mathbb{Q}^{d}$ such that $\mathbf{v}_{0} R \mathbf{v}_{1} \cdots R \mathbf{v}_{k}$ and let $\mu_{1}, \ldots, \mu_{k} \in \mathbb{Q}_{\geq 0}$ such that the following vector $\mathbf{x}_{j}$ is in $\mathbb{Q}_{\geq 0}^{d}$ for every $1 \leq j \leq k$ :

$$
\mathbf{x}_{j}=\left(\mu_{0}-\mu_{1}\right) \mathbf{v}_{0}+\cdots+\left(\mu_{j}-\mu_{j+1}\right) \mathbf{v}_{j}
$$

where $\mu_{0}=1$ and $\mu_{k+1}=0$. Then $\mathbf{v}_{0} R^{n} \mathbf{x}_{k}$ where $n$ is the cardinal of $\left\{j \in\{1, \ldots, k\} \mid \mu_{j}>0\right\}$.

Proof: Let us consider the vector $\mathbf{z}_{j}=\mathbf{x}_{j}+\mu_{j+1} \mathbf{v}_{j}$. As $R$ is reflexive, we deduce that $\left(\mathbf{x}_{j-1}, \mathbf{x}_{j-1}\right) \in R$. Since $R$ is conic, we get $\left(\mathbf{x}_{j-1}, \mathbf{x}_{j-1}\right)+\mu_{j}\left(\mathbf{v}_{j-1}, \mathbf{v}_{j}\right) \in R$. This pair is equal to $\left(\mathbf{z}_{j-1}, \mathbf{z}_{j}\right)$. Thus $\left(\mathbf{z}_{j-1}, \mathbf{z}_{j}\right) \in R$. Since $\mathbf{z}_{j-1}=\mathbf{z}_{j}$ if $\mu_{j}=0$ we deduce that $\mathbf{z}_{0} R^{n} \mathbf{z}_{k}$. Observe that $\mathbf{z}_{0}=\mathbf{x}_{0}+$ $\mu_{1} \mathbf{v}_{0}=\mu_{0} \mathbf{v}_{0}=\mathbf{v}_{0}$ and $\mathbf{z}_{k}=\mathbf{x}_{k}+\mu_{k+1} \mathbf{v}_{k}=\mathbf{x}_{k}$.

Lemma B.4. Let $\mathbf{v}_{0}, \ldots, \mathbf{v}_{k} \in \mathbb{Q}_{\geq 0}^{d}$ and let us consider the sets $I_{j}=\left\{i \in\{1, \ldots, d\} \mid \mathbf{v}_{0}(i)>0 \vee \ldots \vee \mathbf{v}_{j}(i)>0\right\}$. There exist non-negative rational numbers $\mu_{1}, \ldots, \mu_{k} \geq 0$ such that $\mu_{j}=0$ if $I_{j}=I_{j-1}$ and such that for every $0 \leq j \leq k$ :

$$
\left(\mu_{0}-\mu_{1}\right) \mathbf{v}_{0}+\cdots+\left(\mu_{j}-\mu_{j+1}\right) \mathbf{v}_{j} \in \mathbb{Q}_{I_{j}}^{d}
$$

where $\mu_{0}=1$ and $\mu_{k+1}=0$.

Proof: The lemma is immediate with $k=0$. Assume the lemma proved for $k$ and let us consider a sequence $\mathbf{v}_{0}, \ldots, \mathbf{v}_{k+1} \in \mathbb{Q}_{\geq 0}^{d}$ and let us introduce a sequence $\mu_{1}, \ldots, \mu_{k} \geq 0$ such that $\mu_{j}=0$ if $I_{j}=I_{j-1}$ and such that:

$$
\left(\mu_{0}-\mu_{1}\right) \mathbf{v}_{0}+\cdots+\left(\mu_{j}-\mu_{j+1}\right) \mathbf{v}_{j} \in \mathbb{Q}_{I_{j}}^{d}
$$

where $\mu_{0}=1$ and $\mu_{k+1}=0$. Let us consider $\mathbf{x}=$ $\left(\mu_{0}-\mu_{1}\right) \mathbf{v}_{0}+\cdots+\left(\mu_{k}-\mu_{k+1}\right) \mathbf{v}_{k}$. Note that if $I_{k+1}=I_{k}$, by considering $\mu_{k+2}=0$ we are done. So, let us assume that $I_{k+1} \neq I_{k}$. Since $\mathbf{x} \in \mathbb{Q}_{I_{k}}^{d}$ there exists $\epsilon>0$ such that $\mathbf{x}(i)>\epsilon \mathbf{v}_{k}(i)$ for every $i \in I_{k}$. Let us consider the sequence $\left(\mu_{0}^{\prime}, \ldots, \mu_{k+2}^{\prime}\right)=\left(\mu_{0}, \ldots, \mu_{k}, \epsilon, 0\right)$. Observe that $\left(\mu_{0}^{\prime}-\mu_{1}^{\prime}\right) \mathbf{v}_{0}+\cdots+\left(\mu_{j}^{\prime}-\mu_{j+1}^{\prime}\right) \mathbf{v}_{j} \in \mathbb{Q}_{I_{j}}^{d}$ for every $1 \leq j \leq k+1$. We have proved the lemma by induction.

Corollary B.5. Let $R$ be reflexive conic relation over $\mathbb{Q}^{d}$, let $\mathbf{v}_{0}, \ldots, \mathbf{v}_{k} \in \mathbb{Q}^{d}$ such that $\mathbf{v}_{0} R \mathbf{v}_{1} \cdots R \mathbf{v}_{k}$, and let $I=\left\{i \mid \bigvee_{j=0}^{k} \mathbf{v}_{j}(i)>0\right\}$. There exist non-negative rational numbers $\mu_{1}, \ldots, \mu_{k} \geq 0$ such that the following vector $\mathbf{e}$ is in $\mathbb{Q}_{I}^{d}$ and such that $\mathbf{v}_{0} R^{d} \mathbf{e}$ :

$$
\mathbf{e}=\mathbf{v}_{0}+\sum_{j=1}^{k} \mu_{j}\left(\mathbf{v}_{j}-\mathbf{v}_{j-1}\right)
$$

Proof: Let us consider the sets $I_{j}=\{i \in\{1, \ldots, k\} \mid$ $\left.\mathbf{v}_{0}(i)>0 \vee \ldots \vee \mathbf{v}_{j}(i)>0\right\}$. Lemma B.4 shows that there exist non-negative rational numbers $\mu_{1}, \ldots, \mu_{k} \geq 0$ such that $\mu_{j}=0$ if $I_{j}=I_{j-1}$ and such that the following vector $\mathbf{x}_{j}$ is in $\mathbb{Q}_{I_{j}}^{d}$ for every $0 \leq j \leq k$ :

$$
\mathbf{x}_{j}=\left(\mu_{0}-\mu_{1}\right) \mathbf{v}_{0}+\cdots+\left(\mu_{j}-\mu_{j+1}\right) \mathbf{v}_{j}
$$

where $\mu_{0}=1$ and $\mu_{k+1}=0$. Lemma B. 3 shows that $\mathbf{v}_{0} R^{n} \mathbf{x}_{k}$ where $n$ is the cardinal of $\left\{j \in\{1, \ldots, k\} \mid \mu_{j}>0\right\}$. Since 
$n \leq d$ and $R$ is reflexive, we deduce that $R^{n} \subseteq R^{d}$. Note that $\mathbf{e}=\mathbf{x}_{k}$ is in $\mathbb{Q}_{I_{k}}^{d}$. Since $I_{k}=I$, we are done.

Lemma B.6. For every reflexive conic relation $R$ over $\mathbb{Q}_{\geq 0}^{d}$ we have:

$$
R^{+}=R^{d} \circ\left(\sum_{I \subseteq\{1, \ldots, d\}} \nabla_{I}^{-1}\left(\nabla_{I}(R)\right)\right) \circ R^{d}
$$

Proof: From Lemma B.2 we deduce that $\nabla_{I}^{-1}\left(\nabla_{I}(R)\right) \subseteq$ $R^{+}$for every $I \subseteq\{1, \ldots, d\}$. With Lemma B.1 we deduce that $\sum_{I \subset\{1, \ldots, d\}} \nabla_{I}^{-1}\left(\nabla_{I}(R)\right)$ is included in $R^{+}$. We have proved the inclusion $\supseteq$. Let us now prove the inclusion $\subseteq$.

Let us consider $(\mathbf{x}, \mathbf{y}) \in R^{+}$. There exists a sequence $\left(\mathbf{v}_{j}\right)_{0 \leq j \leq k}$ with $k \geq 1$ of vectors $\mathbf{v}_{j} \in \mathbb{Q}_{\geq 0}^{d}$ such that $\mathbf{v}_{0}=\mathbf{x}$, $\mathbf{v}_{k}=\mathbf{y}$ and $\left(\mathbf{v}_{j-1}, \mathbf{v}_{j}\right) \in R$ for every $j \in\{1, \ldots, k\}$. We introduce the set $I=\left\{i \mid \mathbf{v}_{0}(i)>0 \vee \ldots \vee \mathbf{v}_{k}(i)>0\right\}$.

Corollary B.5 shows that there exist $\mu_{1}, \ldots, \mu_{k} \geq 0$ such that $\mathbf{x} R^{d} \mathbf{e}$ where $\mathbf{e}=\mathbf{x}+\sum_{j=1}^{k} \mu_{j}\left(\mathbf{v}_{j}-\mathbf{v}_{j-1}\right)$ is a vector in $\mathbb{Q}_{I}^{d}$. The inverse of $R$ and Corollary B.5 show that there exist $\mu_{1}^{\prime}, \ldots, \mu_{k}^{\prime} \geq 0$ such that $\mathbf{f} R^{d} \mathbf{y}$ where $\mathbf{f}=\mathbf{y}+\sum_{j=1}^{k} \mu_{j}^{\prime}\left(\mathbf{v}_{j-1}-\mathbf{v}_{j}\right)$ is a vector in $\mathbb{Q}_{I}^{d}$.

Let us consider $\mu \geq 0$ such that $\mu-\mu_{j}-\mu_{j}^{\prime} \geq 0$ for every $j$. Let $\mathbf{a}=\sum_{j=1}^{k}\left(1+\mu-\mu_{j}-\mu_{j}^{\prime}\right)\left(\mathbf{v}_{j}-\mathbf{v}_{j-1}\right)$ and let us prove that $\mathbf{a} \in \nabla_{I}(R)$. Let us introduce the vector $\mathbf{e} \in \mathbb{Q}_{I}^{d}$ defined by $\mathbf{e}(i)=1$ if $i \in I$ and $\mathbf{e}(i)=0$ otherwise. Since $R$ is reflexive we get $(\mathbf{e}, \mathbf{e}) \in R$ and since $R$ is conic then $r_{j}=\left(\mathbf{e}+\mathbf{v}_{j-1}, \mathbf{e}+\mathbf{v}_{j}\right)$ is in $R$. Observe that $\mathbf{e}+\mathbf{v}_{j-1}$ and $\mathbf{e}+\mathbf{v}_{j}$ are both in $\mathbb{Q}_{I}^{d}$. We deduce that $\nabla\left(r_{j}\right) \in \nabla_{I}(R)$. Then $\mathbf{v}_{j}-\mathbf{v}_{j-1} \in \nabla_{I}(R)$. Since $\nabla_{I}(R)$ is a conic set we deduce that $\mathbf{a} \in \nabla_{I}(R)$.

We have:

$$
\begin{aligned}
& (\mathbf{f}+\mu \mathbf{y})-(\mathbf{e}+\mu \mathbf{x}) \\
& =(1+\mu) \mathbf{y}-(1+\mu) \mathbf{x}-\sum_{j=1}^{k}\left(\mu_{j}+\mu_{j}^{\prime}\right)\left(\mathbf{v}_{j}-\mathbf{v}_{j-1}\right) \\
& =(1+\mu) \sum_{j=1}^{k}\left(\mathbf{v}_{j}-\mathbf{v}_{j-1}\right)-\sum_{j=1}^{k}\left(\mu_{j}+\mu_{j}^{\prime}\right)\left(\mathbf{v}_{j}-\mathbf{v}_{j-1}\right) \\
& =\sum_{j=1}^{k}\left(1+\mu-\mu_{j}-\mu_{j}^{\prime}\right)\left(\mathbf{v}_{j}-\mathbf{v}_{j-1}\right) \\
& =\mathbf{a}
\end{aligned}
$$

As $\mathbf{a} \in \nabla_{I}(R)$ and $\mathbf{e}+\mu \mathbf{x}, \mathbf{f}+\mu \mathbf{y} \in \mathbb{Q}_{I}^{d}$ we deduce that $\mathbf{e}+\mu \mathbf{x} \nabla_{I}^{-1}\left(\nabla_{I}(R)\right) \mathbf{f}+\mu \mathbf{y}$. From $(\mathbf{x}, \mathbf{e}) \in R^{d}$ and $(\mathbf{x}, \mathbf{x}) \in$ $R^{d}$ and since $R^{d}$ is conic, we deduce that $(1+\mu) \mathbf{x} R^{d} \mathbf{e}+\mu \mathbf{x}$. Symmetrically we get $\mathbf{f}+\mu \mathbf{y} R^{d}(1+\mu) \mathbf{f}$. We have proved that the relation $R^{d} \circ \nabla_{I}^{-1}\left(\nabla_{I}(R)\right) \circ R^{d}$ contains $(1+\mu)(\mathbf{x}, \mathbf{y})$. Since this relation is conic we deduce that it contains $(\mathbf{x}, \mathbf{y})$.

We deduce the proof of Theorem VII.1. 


\section{APPENDIX C}

\section{Proof of LemMA X. 2}

Lemma C.1. Let $\mathbf{P} \subseteq \mathbb{Q}^{d}$ be a periodic set included in $\bigcup_{j=1}^{k} \mathbf{B}_{j}+\mathbf{V}_{j}$ where $k \in \mathbb{N}_{>0}, \mathbf{B}_{j} \subseteq \mathbb{Q}^{d}$ is a bounded set and $\mathbf{V}_{j} \subseteq \mathbb{Q}^{d}$ is a vector space. There exists $j \in\{1, \ldots, k\}$ such that $\mathbf{P} \subseteq \mathbf{V}_{j} \subseteq \mathbf{B}_{j}+\mathbf{V}_{j}$.

Proof: Let us first prove by induction over $k \in \mathbb{N}_{>0}$ that for every periodic set $\mathbf{P} \subseteq \mathbb{Q}^{d}$ included in $\bigcup_{j=1}^{k} \mathbf{V}_{j}$ where $\mathbf{V}_{j} \subseteq \mathbb{Q}^{d}$ is a vector space, there exists $j \in\{1, \ldots, k\}$ such that $\mathbf{P} \subseteq \mathbf{V}_{j}$. The rank $k=1$ is immediate. Let us prove the rank $k+1$ and assume that $\mathbf{P}$ is included in $\bigcup_{j=1}^{k+1} \mathbf{V}_{j}$. If $\mathbf{P} \subseteq \mathbf{V}_{k+1}$ the induction is proved. So we can assume that there exists $\mathbf{p} \in \mathbf{P} \backslash \mathbf{V}_{k+1}$. Let $\mathbf{x} \in \mathbf{P}$. Since $n \mathbf{p}+\mathbf{x} \in \mathbf{P}$ for every $n \in \mathbb{N}$ there exists $j \in\{1, \ldots, k+1\}$ such that $n \mathbf{p}+\mathbf{x} \in \mathbf{V}_{j}$. As $\{1, \ldots, k+1\}$ is finite, there exists $j$ in this set and $n<n^{\prime}$ such that $n \mathbf{p}+\mathbf{x}$ and $n^{\prime} \mathbf{p}+\mathbf{x}$ are both in $\mathbf{V}_{j}$. In particular the difference of this two vectors is in $\mathbf{V}_{j}$. Since this difference is $\left(n^{\prime}-n\right) \mathbf{p}$ and $\mathbf{p} \notin \mathbf{V}_{k+1}$ we get $j \in\{1, \ldots, k\}$. Observe that $n\left(n^{\prime} \mathbf{p}+\mathbf{x}\right)-n^{\prime}(n \mathbf{p}+\mathbf{x})$ is the difference of two vectors in $\mathbf{V}_{j}$. Thus this vector is in $\mathbf{V}_{j}$ and we deduce that $\mathbf{x} \in \mathbf{V}_{j}$. We have shown that $\mathbf{P} \subseteq \bigcup_{j=1}^{k} \mathbf{V}_{j}$. By induction there exists $j \in\{1, \ldots, k\}$ such that $\mathbf{P} \subseteq \mathbf{V}_{j}$. We have proved the induction.

Finally, let $\mathbf{P} \subseteq \mathbb{Q}^{d}$ be a periodic set included in $\bigcup_{j=1}^{k} \mathbf{B}_{j}+$ $\mathbf{V}_{j}$ where $k \in \mathbb{N}_{>0}, \mathbf{B}_{j} \subseteq \mathbb{Q}^{d}$ is a bounded set and $\mathbf{V}_{j} \subseteq \mathbb{Q}^{d}$ is a vector space. Let us consider the set $J$ of $j \in\{1, \ldots, k\}$ such that $\mathbf{V}_{j} \subseteq \mathbf{B}_{j}+\mathbf{V}_{j}$. Let us prove that $\mathbf{P} \subseteq \bigcup_{j \in J} \mathbf{V}_{j}$. Let us consider $\mathbf{p} \in \mathbf{P}$. Since $n \mathbf{p} \in \mathbf{P}$ for every $n \in \mathbb{N}$, the pigeon-hole principle shows that there exists $j \in\{1, \ldots, k\}$ and an infinite set $N \subseteq \mathbb{N}$ such that $n \mathbf{p} \in \mathbf{B}_{j}+\mathbf{V}_{j}$ for every $n \in N$. We deduce that for every $n \in N$ there exists $\mathbf{b}_{n} \in \mathbf{B}_{j}$ such that $n \mathbf{p}-\mathbf{b}_{n} \in \mathbf{V}_{j}$. Lemma V.1 shows that there exists a finite set $\mathbf{H} \subseteq \mathbb{Q}^{d}$ such that $\mathbf{V}_{j}=\left\{\mathbf{v} \in \mathbb{Q}^{d} \mid \bigwedge_{\mathbf{h} \in \mathbf{H}} \mathbf{h} \cdot \mathbf{v}=0\right\}$. Let $\mathbf{h} \in \mathbf{H}$. Since $n \mathbf{p}-\mathbf{b}_{n} \in \mathbf{V}_{j}$ we get $n \mathbf{h} \cdot \mathbf{p}=\mathbf{h} \cdot \mathbf{b}_{n}$ for every $n \in N$. Since $\mathbf{B}_{j}$ is bounded, there exists $c \in \mathbb{Q}_{\geq 0}$ such that $\left|\mathbf{h} \cdot \mathbf{b}_{n}\right| \leq c$ for every $n \in N$. Thus $\mathbf{h} \cdot \mathbf{p}=0$ and we have proved that $\mathbf{p} \in \mathbf{V}_{j}$. From $n \mathbf{p}+\mathbf{b}_{n} \in \mathbf{V}_{j}$ and $\mathbf{p} \in \mathbf{V}_{j}$ we deduce that $\mathbf{b}_{n} \in \mathbf{V}_{j}$. Thus $\mathbf{V}_{j}=\mathbf{b}_{n}+\mathbf{V}_{j} \subseteq \mathbf{B}_{j}+\mathbf{V}_{j}$ and we have proved that $j \in J$. We deduce that $\mathbf{P}$ is included in $\bigcup_{j \in J} \mathbf{V}_{j}$. From the previous paragraph, there exists $j \in J$ such that $\mathbf{P} \subseteq \mathbf{V}_{j}$.

Lemma X.2. We have $\operatorname{dim}(\mathbf{P})=\operatorname{rank}(\mathbf{V})$ for every periodic set $\mathbf{P}$ where $\mathbf{V}$ is the vector space generated by $\mathbf{P}$.

Proof: Since $\mathbf{P} \subseteq \mathbf{V}$ we deduce that $\operatorname{dim}(\mathbf{P}) \leq \operatorname{rank}(\mathbf{V})$. For the converse inequality, there exist $k \in \mathbb{N},\left(\mathbf{B}_{j}\right)_{1 \leq j \leq k}$ a sequence of bounded subsets $\mathbf{B}_{j} \subseteq \mathbb{Q}^{d}$ and a sequence $\mathbf{V}_{j} \subseteq \mathbb{Q}^{d}$ of vector spaces such that $\mathbf{P} \subseteq \bigcup_{j=1}^{k} \mathbf{b}_{j}+\mathbf{V}_{j}$ and such that $\operatorname{rank}\left(\mathbf{V}_{j}\right) \leq \operatorname{dim}(\mathbf{P})$ for every $j$. Since $\mathbf{P}$ is non empty we deduce that $k \in \mathbb{N}_{>0}$. Lemma C.1 proves that there exists $j \in\{1, \ldots, k\}$ such that $\mathbf{P} \subseteq \mathbf{V}_{j}$. By minimality of the vector space generated by $\mathbf{P}$ we get $\mathbf{V} \subseteq \mathbf{V}_{j}$. Hence $\operatorname{rank}(\mathbf{V}) \leq \operatorname{rank}\left(\mathbf{V}_{j}\right)$. From $\operatorname{rank}\left(\mathbf{V}_{j}\right) \leq \operatorname{dim}(\mathbf{P})$ we get $\operatorname{rank}(\mathbf{V}) \leq \operatorname{dim}(\mathbf{P})$ 


\section{APPENDIX D}

\section{AdDitional Results on EQuivalent Sets}

Lemma D.1. Let $\mathbf{V}$ be a vector space and $r=\operatorname{rank}(\mathbf{V})$. For every $\mathbf{h} \in \mathbb{Q}^{d}$ such that $\mathbf{h} \cdot \mathbf{v} \neq 0$ for at least one $\mathbf{v} \in \mathbf{V}$, for every $c \in \mathbb{Q}$ and for every $\# \in\{>, \geq\}$, we have:

$$
\{\mathbf{x} \in \mathbf{V} \mid \mathbf{h} \cdot \mathbf{x} \geq 0\} \equiv_{r}\{\mathbf{x} \in \mathbf{V} \mid \mathbf{h} \cdot \mathbf{x} \# c\}
$$

Proof: Let us introduce a vector $\mathbf{v} \in \mathbf{V}$ such that $\mathbf{h} \cdot \mathbf{v} \neq$ 0 . By replacing $\mathbf{v}$ by $-\mathbf{v}$ we can assume that $\mathbf{h} \cdot \mathbf{v}>0$. We introduce the set $\mathbf{B}=\left\{\lambda \mathbf{v}|| \lambda \mid \leq \frac{|c|}{\mathbf{h} \cdot \mathbf{v}}\right\}$ and the vector space $\mathbf{W}=\{\mathbf{w} \in \mathbf{V} \mid \mathbf{h} \cdot \mathbf{w}=0\}$. Since $\mathbf{W}$ is included in $\mathbf{V} \backslash\{\mathbf{v}\}$ we deduce that $\operatorname{rank}(\mathbf{W})<\operatorname{rank}(\mathbf{V})=r$. Let us prove that the symmetrical difference of $\{\mathbf{x} \in \mathbf{V} \mid \mathbf{h} \cdot \mathbf{x} \geq 0\}$ and $\{\mathbf{x} \in \mathbf{V} \mid \mathbf{h} \cdot \mathbf{x} \# c\}$ is included in $\mathbf{B}+\mathbf{W}$. Let $\mathbf{x}$ be a vector in this difference. Then $\mathbf{x} \in \mathbf{V}$ and either $\mathbf{h} \cdot \mathbf{x} \geq 0$ and $\mathbf{h} \cdot \mathbf{x} \leq c$ or we have $\mathbf{h} \cdot \mathbf{x}<0$ and $\mathbf{h} \cdot \mathbf{x} \geq c$. In any case we deduce that $-|c| \leq \mathbf{h} \cdot \mathbf{x} \leq|c|$. Let us consider $\lambda=\frac{\mathbf{h} \cdot \mathbf{x}}{\mathbf{h} \cdot \mathbf{v}}$. Note that $\mathbf{b}=\lambda \mathbf{v}$ is a vector in $\mathbf{B}$ and $\mathbf{w}=\mathbf{x}-\lambda \mathbf{v}$ is a vector in $\mathbf{W}$. Thus $\mathbf{x} \in \mathbf{B}+\mathbf{W}$.

We deduce the following two corollaries:

Corollary D.2. Let $\mathbf{V}$ be a vector space and $r=\operatorname{rank}(\mathbf{V})$. For every $\mathbf{X} \subseteq \mathbf{V}$ definable in $\mathrm{FO}(\mathbb{Q},+, \leq, 0,1)$ and for every $\mathbf{v} \in \mathbf{V}$ we have $\mathbf{X} \equiv{ }_{r} \mathbf{v}+\mathbf{X}$.

Proof: Since $\mathrm{FO}(\mathbb{Q},+, \leq, 0,1)$ admits quantifier elimination we deduce that $\mathbf{X}$ is a Boolean (union and intersection) combination of sets of the form $\mathbf{S}=\{\mathbf{s} \in \mathbf{V} \mid \mathbf{h} \cdot \mathbf{s} \# c\}$ where $\mathbf{h} \in \mathbb{Q}^{d}$, \# $\in\{>, \geq\}$, and $c \in \mathbb{Q}$. Note that $\mathbf{v}+\mathbf{S}=\{\mathbf{s} \in \mathbf{V} \mid \mathbf{h} \cdot \mathbf{s} \# \mathbf{h} \cdot \mathbf{v}\}$. In particular if $\mathbf{h} \cdot \mathbf{v}=0$ then $\mathbf{S}=\mathbf{S}+\mathbf{v}$ and if $\mathbf{h} \cdot \mathbf{v} \neq 0$ Lemma D.1 shows that $\mathbf{S} \equiv_{r} \mathbf{v}+\mathbf{S}$. We deduce that $\mathbf{S} \equiv_{r} \mathbf{v}+\mathbf{S}$ is both case. Since $\equiv_{r}$ is distributive over $\cup$ and $\cap$ we get the corollary.

Corollary D.3. Let $\mathbf{P} \subseteq \mathbb{Z}^{d}$ be a finitely generated periodic set, $\mathbf{L}=\mathbf{P}-\mathbf{P}$ the lattice generated by $\mathbf{P}$, and $\mathbf{C}=\mathbb{Q}_{\geq 0} \mathbf{P}$ be the conic set generated by $\mathbf{P}$. For every $\mathrm{x} \in \mathbf{L}$ we have $\mathbf{x}+\mathbf{P} \equiv_{r} \mathbf{L} \cap \mathbf{C}$ where $r=\operatorname{dim}(\mathbf{P})$.

Proof: Since $\mathbf{P}$ is finitely generated, there exists $\mathbf{p}_{1}, \ldots, \mathbf{p}_{k} \in \mathbf{P}$ such that $\mathbf{P}=\mathbb{N} \mathbf{p}_{1}+\cdots+\mathbb{N} \mathbf{p}_{k}$. We introduce the set $\mathbf{B}$ of vectors $\mathbf{b} \in \mathbf{L}$ such that $\mathbf{b} \in$ $[0,1] \mathbf{p}_{1}+\cdots+[0,1] \mathbf{p}_{k}$. Note that $\mathbf{B}$ is a bounded finite subset of $\mathbb{Z}^{d}$. Thus $\mathbf{B}$ is finite. Since $\mathbf{B} \subseteq \mathbf{P}-\mathbf{P}$ we deduce that there exists $\mathbf{p} \in \mathbf{P}$ such that $\mathbf{p}+\mathbf{b} \in \mathbf{P}$ for every $\mathbf{b} \in \mathbf{B}$. Let us prove that $\mathbf{p}+(\mathbf{L} \cap \mathbf{C}) \subseteq \mathbf{P}$. Let us consider $\mathbf{v} \in \mathbf{L} \cap \mathbf{C}$. There exists a sequence $\mu_{1}, \ldots, \mu_{k} \in \mathbb{Q}_{\geq 0}$ such that $\mathbf{v}=\mu_{1} \mathbf{p}_{1}+\cdots+\mu_{k} \mathbf{v}_{k}$. Let $n_{j} \in \mathbb{N}$ such that $\mu_{j}-n_{j} \in[0,1]$ and let $\mathbf{q}=n_{1} \mathbf{p}_{1}+\cdots+n_{k} \mathbf{p}_{k}$. Note that $\mathbf{q} \in \mathbf{P}$ and $\mathbf{v}-\mathbf{q} \in \mathbf{B}$. Thus $\mathbf{p}+\mathbf{v}-\mathbf{q} \in \mathbf{P}$. In particular $\mathbf{p}+\mathbf{v} \in \mathbf{P}$ and we have proved the inclusion $\mathbf{p}+(\mathbf{L} \cap \mathbf{C}) \subseteq \mathbf{P}$. Since $\mathbf{p} \in \mathbf{L}$ we get $\mathbf{p}+\mathbf{L}=\mathbf{L}$. Thus $\mathbf{p}+(\mathbf{L} \cap \mathbf{C})=$ $\mathbf{L} \cap(\mathbf{p}+\mathbf{C})$. Corollary D. 2 shows that $\mathbf{C} \equiv_{r} \mathbf{p}+\mathbf{C}$. Since $\equiv_{r}$ is distributive over the intersection, we get $\mathbf{L} \cap(\mathbf{p}+\mathbf{C}) \equiv_{r} \mathbf{L} \cap \mathbf{C}$. Moreover, from $\mathbf{L} \cap(\mathbf{p}+\mathbf{C}) \subseteq \mathbf{P} \subseteq \mathbf{L} \cap \mathbf{C}$ we deduce that $\mathbf{P} \equiv_{r} \mathbf{L} \cap \mathbf{C}$. Note that for every $\mathbf{x} \in \mathbf{L}$ we have $-\mathbf{X}+(\mathbf{L} \cap \mathbf{C})=\mathbf{L} \cap(-\mathbf{x}+\mathbf{C}) \equiv_{r} \mathbf{L} \cap \mathbf{C}$ thanks to corollary D.2. We have proved that $\mathbf{x}+\mathbf{P} \equiv{ }_{r} \mathbf{L} \cap \mathbf{C}$ for every $\mathbf{x} \in \mathbf{L}$. 


\section{APPENDIX E}

\section{COMPLETE EXTRACTIONS}

Let $\mathcal{K}$ be a finite class of definable conic sets of $\mathbb{Q}^{d}$. We denote by $\Sigma(\mathcal{K})$ the set $\bigcup_{\mathbf{K} \in \mathcal{K}} \mathbf{K}$. An extraction of $\mathcal{K}$ is a finite class $\mathcal{C}$ of finitely generated conic sets of $\mathbb{Q}^{d}$ such that for every $\mathbf{C} \in \mathcal{C}$ there exists $\mathbf{K} \in \mathcal{K}$ such that $\mathbf{C} \subseteq \mathbf{K}$. An extraction $\mathcal{C}$ of $\mathcal{K}$ is said to be complete if $\Sigma(\mathcal{C})=\Sigma(\mathcal{K})$.

Example E.1. Let us consider the class $\mathcal{K}=\left\{\mathbf{K}_{1}, \mathbf{K}_{2}\right\}$ with $\mathbf{K}_{1}=\{\mathbf{0}\} \cup\left(\mathbb{Q} \times \mathbb{Q}_{>0}\right)$ and $\mathbf{K}_{2}=\mathbb{Q} \times \mathbb{Q}<0$. Observe that $\Sigma(\mathcal{K})$ is equal to $\mathbb{Q}^{2}$. We show that there does not exist a complete extraction of $\mathcal{K}$ as follow. We first consider a finitely generated conic set $\mathbf{C}$ included in $\mathbf{K}_{1}$. Such a conic set is generated by a finite set of vectors in $\mathbf{K}_{1} \backslash\{\mathbf{0}\}=\mathbb{Q} \times \mathbb{Q}>0$. So there exists $\epsilon \in \mathbb{Q}_{>0}$ such that $\mathbf{C} \subseteq \mathbb{Q}_{\geq 0}(1, \epsilon)+\mathbb{Q}_{\geq 0}(-1, \epsilon)$. Now, let us consider an extraction $\mathcal{C}$ of $\mathcal{K}$. We have proved that there exists $\epsilon \in \mathbb{Q}_{>0}$ such that $\Sigma(\mathcal{C}) \subseteq\left(\mathbb{Q}_{>0}(1, \epsilon)+\right.$ $\mathbb{Q} \geq 0(-1, \epsilon)) \cup(\mathbb{Q} \times \mathbb{Q} \leq 0)$ which is strictly included in $\mathbb{Q}^{2}$ (for instance $\left(1, \frac{\epsilon}{2}\right)$ is not in this set).

In this section finite classes $\mathcal{K}$ of definable conic sets of $\mathbb{Q}^{d}$ having a complete extraction are topologically characterized thanks to the overlapping property ${ }^{1}$. The class $\mathcal{K}$ is said to have the overlapping property if for every $\mathbf{K} \in \mathcal{K}$ and for every finite sequence $\mathbf{v}_{1}, \ldots, \mathbf{v}_{k}$ of vectors $\mathbf{v}_{j} \in \mathbb{Q}^{d}$ satisfying $\mathbb{Q}_{>0} \mathbf{v}_{1}+\cdots+\mathbb{Q}_{>0} \mathbf{v}_{k} \subseteq \mathbf{K}$ there exists $\mathbf{K}^{\prime} \in \mathcal{K}$ such that $\mathbf{K}^{\prime} \cap\left(\mathbb{Q}_{>0} \mathbf{v}_{1}+\cdots+\mathbb{Q}_{>0} \mathbf{v}_{j}\right)$ is non empty for every $j \in$ $\{1, \ldots, k\}$. We are going to prove the following result:

Theorem E.2. A finite class $\mathcal{K}$ of definable conic sets of $\mathbb{Q}^{d}$ has the overlapping property if and only if it has the complete extraction property.

Example E.3. Let us come back to the class $\mathcal{K}=\left\{\mathbf{K}_{1}, \mathbf{K}_{2}\right\}$ with $\mathbf{K}_{1}=\{\mathbf{0}\} \cup\left(\mathbb{Q} \times \mathbb{Q}_{>0}\right)$ and $\mathbf{K}_{2}=\mathbb{Q} \times \mathbb{Q}<0$ introduced in Example E.1. We show that $\mathcal{K}$ does not satisfy the overlapping property by considering the sequence $\mathbf{v}_{1}, \mathbf{v}_{2}$ defined by $\mathbf{v}_{1}=(1,0)$ and $\mathbf{v}_{2}=(1,1)$. Now, just observe that $\mathbb{Q}_{>0} \mathbf{v}_{1}+\mathbb{Q}_{>0} \mathbf{v}_{2} \subseteq \mathbf{K}_{1}$ but $\mathbf{K}_{1} \cap\left(\mathbb{Q}_{>0} \mathbf{v}_{1}\right)$ and $\mathbf{K}_{2} \cap\left(\mathbb{Q}_{>0} \mathbf{v}_{1}+\mathbb{Q}_{>0} \mathbf{v}_{2}\right)$ are empty.

We observe that if a finite class $\mathcal{K}$ of definable conic sets of $\mathbb{Q}^{d}$ has a complete extraction $\mathcal{C}$, then for every $\mathbf{K} \in \mathcal{K}$ and for every sequence $\mathbf{v}_{1}, \ldots, \mathbf{v}_{k}$ of vectors $\mathbf{v}_{j} \in \mathbb{Q}^{d}$ such that $\mathbb{Q}_{>0} \mathbf{v}_{1}+\cdots+\mathbb{Q}_{>0} \mathbf{v}_{k} \subseteq \mathbf{K}$, from $\mathbf{K} \subseteq \bigcup_{\mathbf{C} \in \mathcal{C}} \mathbf{C}$, the following lemma shows that there exists $\mathbf{C} \in \mathcal{C}$ such that $\mathbf{C} \cap\left(\mathbb{Q}_{>0} \mathbf{v}_{1}+\right.$ $\left.\cdots+\mathbb{Q}_{>0} \mathbf{v}_{j}\right) \neq \emptyset$ for every $j \in\{1, \ldots, k\}$. Since $\mathcal{C}$ is an extraction of $\mathcal{K}$ we deduce that there exists $\mathbf{K}^{\prime} \in \mathcal{K}$ such that $\mathbf{C} \subseteq \mathbf{K}^{\prime}$. Therefore $\mathbf{K}^{\prime} \cap\left(\mathbb{Q}_{>0} \mathbf{v}_{1}+\cdots+\mathbb{Q}_{>0} \mathbf{v}_{j}\right) \neq \emptyset$ for every $j \in\{1, \ldots, k\}$. We have proved that $\mathcal{K}$ has the overlapping property.

Lemma E.4. For every sequence $\mathbf{v}_{1}, \ldots, \mathbf{v}_{k}$ of vectors $\mathbf{v}_{j} \in$ $\mathbb{Q}^{d}$ and for every finite class $\mathcal{C}$ of finitely generated conic sets of $\mathbb{Q}^{d}$ such that $\mathbb{Q}_{>0} \mathbf{v}_{1}+\cdots+\mathbb{Q}_{>0} \mathbf{v}_{k} \subseteq \bigcup_{\mathbf{C} \in \mathcal{C}} \mathbf{C}$, there exists

\footnotetext{
${ }^{1}$ The term "overlapping" comes from a topological property introduced by Lambert in an unpublished work similar to the one we consider in this paper
}

$\mathbf{C} \in \mathcal{C}$ such that $\mathbf{C} \cap\left(\mathbb{Q}_{>0} \mathbf{v}_{1}+\cdots+\mathbb{Q}_{>0} \mathbf{v}_{j}\right) \neq \emptyset$ for every $j \in\{1, \ldots, k\}$.

Proof: We prove the lemma by induction over $k \in \mathbb{N}_{>0}$. The rank $k=1$ is immediate since from $\mathbb{Q}_{>0} \mathbf{v}_{1} \subseteq \bigcup_{\mathbf{C} \in \mathcal{C}} \mathbf{C}$ we deduce that there exists $\mathbf{C} \in \mathcal{C}$ such that $\mathbf{C} \cap\left(\mathbb{Q}_{>0} \mathbf{v}_{1}\right)$ is non empty. Let us assume the induction proved for a rank $k \in \mathbb{N}_{>0}$ and let us consider a sequence $\mathbf{v}_{0}, \ldots, \mathbf{v}_{k}$ of vectors in $\mathbb{Q}^{d}$ and a finite class $\mathcal{C}$ of finitely generated conic sets of $\mathbb{Q}^{d}$ such that $\mathbb{Q}_{>0} \mathbf{v}_{0}+\cdots+\mathbb{Q}_{>0} \mathbf{v}_{k} \subseteq \bigcup_{\mathbf{C} \in \mathcal{C}} \mathbf{C}$. We introduce the finite class $\mathcal{C}_{0}=\left\{\mathbf{C} \in \mathcal{C} \mid \mathbf{v}_{0} \in \mathbf{C}\right\}$. We are going to prove that there exists a sequence $\left(\lambda_{j}\right)_{1<j<k}$ of rational numbers $\lambda_{j} \in \mathbb{Q}_{>0}$ such that $\mathbb{Q}_{>0}\left(\mathbf{v}_{1}+\lambda_{1} \mathbf{v}_{0}\right)+\cdots+\mathbb{Q}_{>0}\left(\mathbf{v}_{k}+\lambda_{k} \mathbf{v}_{0}\right) \subseteq$ $\bigcup_{\mathbf{C} \in \mathcal{C}_{0}} \mathbf{C}$.

Since every $\mathbf{C} \in \mathcal{C}$ is a finitely generated conic set, Lemma V.2 shows that there exists a finite set $\mathbf{H}_{\mathbf{C}} \subseteq \mathbb{Q}^{d}$ such that:

$$
\mathbf{C}=\bigcap_{\mathbf{h} \in \mathbf{H}_{\mathbf{C}}}\left\{\mathbf{v} \in \mathbb{Q}^{d} \mid \mathbf{h} \cdot \mathbf{v} \geq 0\right\}
$$

We introduce the set $\mathbf{H}=\bigcup_{\mathbf{C} \in \mathcal{C}} \mathbf{H}_{\mathbf{C}}$ and the set $\mathbf{H}_{0}=\{\mathbf{h} \in$ $\left.\mathbf{H} \mid \mathbf{h} \cdot \mathbf{v}_{0}>0\right\}$.

We build up a sequence $\left(\lambda_{j}\right)_{1 \leq j \leq k}$ of rational numbers $\lambda_{j} \in$ $\mathbb{Q}_{>0}$ such that $\mathbf{h} \cdot\left(\mathbf{v}_{j}+\lambda_{j} \mathbf{v}_{0}\right) \geq 0$ for every $\mathbf{h} \in \mathbf{H}_{0}$ as follows. Let $\mathbf{h} \in \mathbf{H}_{0}$ and $j \in\{1, \ldots, k\}$. Since $\mathbf{h} \cdot \mathbf{v}_{0}>0$ we deduce that there exists $\lambda_{\mathbf{h}, j} \in \mathbb{Q}_{>0}$ such that $\mathbf{h} \cdot\left(\mathbf{v}_{j}+\right.$ $\left.\lambda_{\mathbf{h}, j} \mathbf{v}_{0}\right) \geq 0$. We introduce a rational number $\lambda_{j} \in \mathbb{Q}>0$ such that $\lambda_{j} \geq \lambda_{\mathbf{h}, j}$ for every $\mathbf{h} \in \mathbf{H}_{0}$. By construction observe that $\mathbf{h} \cdot\left(\mathbf{v}_{j}+\lambda_{j} \mathbf{v}_{0}\right) \geq 0$ for every $\mathbf{h} \in \mathbf{H}_{0}$ and for every $j \in\{1, \ldots, k\}$.

We introduce the sequence $\left(\mathbf{w}_{j}\right)_{1 \leq j \leq k}$ of vectors $\mathbf{w}_{j}=$ $\mathbf{v}_{j}+\lambda_{j} \mathbf{v}_{0}$. Now, let us consider $\mathbf{x} \in \mathbb{Q}>0 \mathbf{w}_{1}+\cdots+\mathbb{Q}_{>0} \mathbf{w}_{k}$ and let us prove that $\mathbf{x} \in \bigcup_{\mathbf{C} \in \mathcal{C}_{0}} \mathbf{C}$. Observe that for every $n \in \mathbb{N}$ we have $n \mathbf{v}_{0}+\mathbf{x} \in \mathbb{Q}_{>0} \mathbf{v}_{0}+\cdots+\mathbb{Q}_{>0} \mathbf{v}_{k} \subseteq \bigcup_{\mathbf{C} \in \mathcal{C}} \mathbf{C}$. Hence there exists $\mathbf{C}_{n} \in \mathcal{C}$ such that $n \mathbf{v}_{0}+\mathbf{x} \in \mathbf{C}_{n}$. Since $\mathcal{C}$ is finite, there exists $\mathbf{C} \in \mathcal{C}$ such that $\mathbf{C}_{n}=\mathbf{C}$ for an infinite number of $n \in \mathbb{N}$. Let $\mathbf{h} \in \mathbf{H}_{\mathbf{C}}$. Since $n \mathbf{v}_{0}+\mathbf{x} \in \mathbf{C}$ we get $n \mathbf{h} \cdot \mathbf{v}_{0}+\mathbf{h} \cdot \mathbf{x} \geq 0$. As this inequality holds for an infinite number of $n \in \mathbb{N}$ we deduce that $\mathbf{h} \cdot \mathbf{v}_{0} \geq 0$. In particular $\mathbf{v}_{0} \in \mathbf{C}$ and we deduce that $\mathbf{C} \in \mathcal{C}_{0}$. Note that if $\mathbf{h} \cdot \mathbf{v}_{0}=0$ then $\mathbf{h} \cdot \mathbf{x} \geq 0$. Otherwise, if $\mathbf{h} \cdot \mathbf{v}_{0}>0$ then $\mathbf{h} \in \mathbf{H}_{0}$. In this case $\mathbf{h} \cdot \mathbf{w}_{j} \geq 0$ for every $j$. From $\mathbf{x} \in \mathbb{Q}>0 \mathbf{w}_{1}+\cdots+\mathbb{Q}_{>0} \mathbf{w}_{k}$ we get $\mathbf{h} \cdot \mathbf{x} \geq 0$. We have proved that $\mathbf{h} \cdot \mathbf{x} \geq 0$ for every $\mathbf{h} \in \mathbf{H}_{\mathbf{C}}$. Therefore $\mathbf{x} \in \mathbf{C}$ and we have proved the inclusion $\mathbb{Q}_{>0} \mathbf{w}_{1}+\cdots+\mathbb{Q}_{>0} \mathbf{w}_{k} \subseteq \bigcup_{\mathbf{C} \in \mathcal{C}_{0}} \mathbf{C .}$

By induction, there exists $\mathbf{C} \in \mathcal{C}_{0}$ such that $\mathbf{C} \cap\left(\mathbb{Q}_{>0} \mathbf{w}_{1}+\right.$ $\left.\cdots+\mathbb{Q}_{>0} \mathbf{w}_{j}\right)$ is non empty for every $j \in\{1, \ldots, k\}$. Since $\mathbb{Q}>0 \mathbf{w}_{1}+\cdots+\mathbb{Q}_{>0} \mathbf{w}_{k} \subseteq \mathbb{Q}_{>0} \mathbf{v}_{0}+\cdots+\mathbb{Q}_{>0} \mathbf{v}_{j}$ we deduce that $\mathbf{C} \cap\left(\mathbb{Q}_{>0} \mathbf{v}_{0}+\cdots+\mathbb{Q}_{>0} \mathbf{v}_{j}\right)$ is non empty for every $j \in\{1, \ldots, k\}$. As $\mathbf{C} \cap\left(\mathbb{Q}_{>0} \mathbf{v}_{0}\right)$ contains $\mathbf{v}_{0}$, this set is also non empty. Therefore, we have proved the induction at rank $k+1$.

Given a finitely generated conic set $\mathbf{C} \subseteq \mathbb{Q}^{d}$ and a finite class $\mathcal{K}$ of definable conic sets, we denote by $\mathbf{C} \cap \mathcal{K}$ the finite class $\{\mathbf{C} \cap \mathbf{K} \mid \mathbf{K} \in \mathcal{K}\}$. 
Lemma E.5. For every finite class $\mathcal{K}$ of definable conic sets of $\mathbb{Q}^{d}$ with the overlapping property and for every finitely generated conic set $\mathbf{C} \subseteq \mathbb{Q}^{d}$, the class $\mathbf{C} \cap \mathcal{K}$ has the overlapping property.

Proof: Let us consider $\mathbf{K} \in \mathcal{K}$ and a sequence $\mathbf{c}_{1}, \ldots, \mathbf{c}_{k}$ of vector $\mathbf{c}_{j} \in \mathbb{Q}^{d}$ such that $\mathbb{Q}_{>0} \mathbf{c}_{1}+\cdots+\mathbb{Q}_{>0} \mathbf{c}_{k} \subseteq \mathbf{C} \cap$ $\mathbf{K}$. Since $\mathcal{K}$ has the overlapping property, there exists $\mathbf{K}^{\prime} \in$ $\mathcal{K}$ such that $\mathbf{K}^{\prime} \cap\left(\mathbb{Q}_{>0} \mathbf{c}_{1}+\cdots+\mathbb{Q}_{>0} \mathbf{c}_{j}\right)$ is non empty for every $j \in\{1, \ldots, k\}$. As $\mathbf{C}$ is a finitely generated conic set, Lemma V.2 shows that there exists a finite set $\mathbf{H} \subseteq \mathbb{Q}^{d}$ such that:

$$
\mathbf{C}=\left\{\mathbf{c} \in \mathbb{Q}^{d} \mid \bigwedge_{\mathbf{h} \in \mathbf{H}} \mathbf{h} \cdot \mathbf{v} \geq 0\right\}
$$

Let $\mathbf{c}=\sum_{j=1}^{k} \mathbf{c}_{j}$. As $\mathbb{Q}_{>0} \mathbf{c}_{1}+\cdots+\mathbb{Q}_{>0} \mathbf{c}_{k} \subseteq \mathbf{C}$ we deduce that $\mathbf{c}+\mathbb{Q} \geq 0$ c $\mathbf{c}_{j} \subseteq \mathbf{C}$. In particular $\mathbf{h} \cdot \mathbf{c}+\lambda \mathbf{h} \cdot \mathbf{c}_{j} \geq 0$ for every $\lambda \in \mathbb{Q}_{\geq 0}$. Thus $\mathbf{h} \cdot \mathbf{c}_{j} \geq 0$. We deduce that $\mathbf{c}_{j} \in \mathbf{C}$. Hence $\mathbb{Q}_{>0} \mathbf{c}_{1}+\cdots+\mathbb{Q}_{>0} \mathbf{c}_{j} \subseteq \mathbf{C}$ for every $j \in\{1, \ldots, k\}$. In particular $\mathbf{C} \cap \mathbf{K}^{\prime} \cap\left(\mathbb{Q}_{>0} \mathbf{c}_{1}+\cdots+\mathbb{Q}_{>0} \mathbf{c}_{j}\right)$ is non empty for every $j \in\{1, \ldots, k\}$. We have proved that the class $\mathbf{C} \cap \mathcal{K}$ has the overlapping property.

Lemma E.6. Let $\mathcal{K}$ be a finite class of definable conic sets of $\mathbb{Q}^{d}$ with the overlapping property then $\mathcal{K}$ has the complete extraction property.

Proof: We prove by induction over $r \in \mathbb{N}$ that for every vector space $\mathbf{V} \subseteq \mathbb{Q}^{d}$ with $\operatorname{rank}(\mathbf{V}) \leq r$ and for every finite class $\mathcal{K}$ of definable conic subsets of $\mathbf{V}$, if $\mathcal{K}$ has the overlapping property then it has the complete extraction property. The rank $r=0$ is immediate since in this case $\mathbf{V}=\{\mathbf{0}\}$. So, let us assume the induction proved for a rank $r \in \mathbb{N}$ and let us consider a vector space $\mathbf{V} \subseteq \mathbb{Q}^{d}$ with $\operatorname{rank}(\mathbf{V}) \leq r+1$ and a finite class $\mathcal{K}$ of definable conic subsets of $\mathbf{V}$. We assume that $\mathcal{K}$ has the overlapping property.

Since $\mathcal{K}$ is a finite class of sets definable in $\mathrm{FO}(\mathbb{Q},+, \leq, 0)$, and this logic admits a quantifier elimination algorithm, we deduce that there exists a finite set $\mathbf{H} \subseteq \mathbb{Q}^{d}$ such that every $\mathbf{K} \in \mathcal{K}$ is the set of vectors $\mathbf{v} \in \mathbf{V}$ satisfying a boolean combination of constraints of the form $\mathbf{h} \cdot \mathbf{x} \# 0$ where $\# \in$ $\{<, \leq, \geq,>\}$. Note that if a vector $\mathbf{h} \in \mathbf{H}$ satisfies $\mathbf{h} \cdot \mathbf{v}=0$ for every $\mathbf{v} \in \mathbf{V}$ then the constraints $\mathbf{h} \cdot \mathbf{x} \# 0$ is useless. So, we can assume without loss of generality that for every $\mathbf{h} \in \mathbf{H}$ there exists $\mathbf{v} \in \mathbf{V}$ such that $\mathbf{h} \cdot \mathbf{v} \neq 0$.

Let us consider for every $s: \mathbf{H} \rightarrow\{-1,1\}$ the finitely generated conic set $\mathbf{C}_{s}=\{\mathbf{v} \in \mathbf{V} \mid s(\mathbf{h}) \mathbf{h} \cdot \mathbf{v} \geq 0\}$. Since $\mathcal{K}$ has the overlapping property, LemmaE.5 shows that $\mathcal{K}_{s}=$ $\mathbf{C}_{s} \cap \mathcal{K}$ has the overlapping property. From $\mathbf{V}=\bigcup_{s} \mathbf{C}_{s}$ we deduce that $\Sigma(\mathcal{K})=\bigcup_{s} \Sigma\left(\mathcal{K}_{s}\right)$. So, it is sufficient to prove that $\mathcal{K}_{s}$ has the complete extraction property. By replacing $\mathcal{K}$ by $\mathcal{K}_{s}$ and $\mathbf{H}$ by $\{s(\mathbf{h}) \mathbf{h} \mid \mathbf{h} \in \mathbf{H}\}$, we can assume without loss of generality that $\mathbf{h} \cdot \mathbf{v} \geq 0$ for every $\mathbf{v} \in \Sigma(\mathcal{K})$.

We introduce the following finitely generated conic set $\mathbf{C}$ and the following set $\mathbf{X}$ :

$$
\begin{aligned}
& \mathbf{C}=\bigcap_{\mathbf{h} \in \mathbf{H}_{0}}\{\mathbf{c} \in \mathbf{V} \mid \mathbf{h} \cdot \mathbf{c} \geq 0\} \\
& \mathbf{X}=\bigcap_{\mathbf{h} \in \mathbf{H}_{0}}\{\mathbf{x} \in \mathbf{V} \mid \mathbf{h} \cdot \mathbf{x}>0\}
\end{aligned}
$$

We also introduce for every $\mathbf{h} \in \mathbf{H}$ the vector space $\mathbf{V}_{\mathbf{h}}=$ $\{\mathbf{v} \in \mathbf{V} \mid \mathbf{h} \cdot \mathbf{v}=0\}$. Since for every $\mathbf{h} \in \mathbf{H}$ there exists a vector $\mathbf{v} \in \mathbf{V}$ such that $\mathbf{h} \cdot \mathbf{v} \neq 0$ we deduce that $\mathbf{V}_{\mathbf{h}}$ is strictly included in $\mathbf{V}$ and in particular $\operatorname{rank}\left(\mathbf{V}_{\mathbf{h}}\right) \leq r$. Lemma E.5 shows that $\mathbf{V}_{\mathbf{h}} \cap \mathcal{K}$ has the overlapping property and by induction we deduce that this class has the complete extraction property. We introduce the set $\mathcal{K}^{\prime}=\{\mathbf{K} \in \mathcal{K} \mid$ $\mathbf{K} \cap \mathbf{X} \neq \emptyset\}$. Since $\mathbf{C} \backslash \mathbf{X}$ is included in $\bigcup_{\mathbf{h} \in \mathbf{H}} \mathbf{V}_{\mathbf{h}}$ we deduce that $\Sigma(\mathcal{K})$ is equal to the union of the sets $\Sigma\left(\mathbf{V}_{\mathbf{h}} \cap \mathcal{K}\right)$ indexed by $\mathbf{h} \in \mathbf{H}$ and $\Sigma\left(\mathcal{K}^{\prime}\right)$. Therefore, in order to prove that $\mathcal{K}$ has the complete extraction property it is sufficient to prove that $\mathcal{K}^{\prime}$ has the complete extraction property is immediate.

Let us prove that $\mathbf{X} \subseteq \mathbf{K}$ for every $\mathbf{K} \in \mathcal{K}^{\prime}$. Recall that $\mathbf{K}$ is the set of vectors $\mathbf{v} \in \mathbf{V}$ satisfying a boolean combination of constraints of the form $\mathbf{h} \cdot \mathbf{x} \# 0$ where $\# \in\{<, \leq, \geq,>\}$. As $\mathbf{K} \cap \mathbf{X}$ is non empty we deduce that this boolean combination is true when the predicates $\mathbf{h} \cdot \mathbf{x} \# 0$ with $\# \in\{\geq,>\}$ are evaluated to true. We deduce that $\mathbf{X} \subseteq \mathbf{K}$.

Let us prove that $\mathcal{K}^{\prime}$ has the overlapping property. Let us consider $\mathbf{K} \in \mathcal{K}^{\prime}$ and a sequence $\mathbf{v}_{1}, \ldots, \mathbf{v}_{k}$ of vectors in $\mathbb{Q}^{d}$ such that $\mathbb{Q}_{>0} \mathbf{v}_{1}+\cdots+\mathbb{Q}_{>0} \mathbf{v}_{k} \subseteq \mathbf{K}$. Since $\mathbf{K} \cap \mathbf{X}$ is non empty, there exists a vector $\mathbf{x}$ in this intersection. As $\mathbf{X} \subseteq \mathbf{K}$ we deduce that $\mathbb{Q}_{>0} \mathbf{v}_{1}+\cdots+\mathbb{Q}_{>0} \mathbf{v}_{k}+\mathbb{Q}_{>0} \mathbf{x} \subseteq \mathbf{X} \subseteq \mathbf{K}$. As $\mathcal{K}$ has the overlapping property we deduce that there exists $\mathbf{K}^{\prime} \in \mathcal{K}$ such that $\mathbf{K}^{\prime} \cap\left(\mathbb{Q}_{>0} \mathbf{v}_{1}+\cdots+\mathbb{Q}_{>0} \mathbf{v}_{k}+\mathbb{Q}_{>0} \mathbf{x}\right)$ is non empty and such that $\mathbf{K}^{\prime} \cap\left(\mathbb{Q}_{>0} \mathbf{v}_{1}+\cdots+\mathbb{Q}_{>0} \mathbf{v}_{j}\right)$ is non empty for every $j \in\{1, \ldots, k\}$. Since $\mathbb{Q}_{>0} \mathbf{v}_{1}+\cdots+\mathbb{Q}>0 \mathbf{v}_{k}+$ $\mathbb{Q}_{>0} \mathbf{X}_{s} \subseteq \mathbf{X}_{s}$ we deduce that $\mathbf{K}^{\prime} \in \mathcal{K}^{\prime}$. Therefore $\mathcal{K}^{\prime}$ has the overlapping property.

Note that if $\mathbf{X}$ is empty then $\mathcal{K}^{\prime}$ has a complete extraction. So, we can assume that $\mathbf{X}$ is non empty. We fix $\mathbf{x} \in \mathbf{X}$. Lemma E.5 shows that $\mathbf{V}_{\mathbf{h}} \cap \mathcal{K}^{\prime}$ has the overlapping property for every $\mathbf{h} \in \mathbf{H}$. By induction we deduce that this class has the complete extraction property. We denote by $\mathcal{C}_{\mathbf{h}}$ a complete extraction of $\mathbf{V}_{\mathbf{h}} \cap \mathcal{K}^{\prime}$ and we consider the following class $\mathcal{C}$ :

$$
\mathcal{C}=\left\{\mathbf{C}+\mathbb{Q}_{\geq 0} \mathbf{x} \mid \mathbf{C} \in \bigcup_{\mathbf{h} \in \mathbf{H}} \mathcal{C}_{\mathbf{h}}\right\}
$$

Let us first prove that $\mathcal{C}$ is an extraction of $\mathcal{K}^{\prime}$. Let $\mathbf{h} \in \mathbf{H}$ and $\mathbf{C} \in \mathcal{C}_{\mathbf{h}}$. Since $\mathcal{C}_{\mathbf{h}}$ is an extraction of $\mathbf{V}_{\mathbf{h}} \cap \mathcal{K}_{s}$ we deduce that there exists $\mathbf{K} \in \mathcal{K}^{\prime}$ such that $\mathbf{C} \subseteq \mathbf{V}_{\mathbf{h}} \cap \mathbf{K}$. Let $\lambda \in \mathbb{Q}_{>0}$ and observe that $\mathbf{C}+\lambda \mathbf{x} \subseteq \mathbf{X} \subseteq \mathbf{K}$. Hence $\mathbf{C}+\mathbb{Q}_{\geq 0} \mathbf{x} \subseteq \mathbf{K}$. We have proved that $\mathcal{C}$ is an extraction of $\mathcal{K}^{\prime}$.

Let us prove that the completeness of the extraction $\mathcal{C}$ of $\mathcal{K}^{\prime}$. We consider $\mathbf{y} \in \Sigma\left(\mathcal{K}^{\prime}\right)$. Since $\mathbf{x} \in \mathbf{X}$ we deduce that $\mathbf{h} \cdot \mathbf{x}>0$ and since $\mathbf{y} \in \mathbf{C}$ we get $\mathbf{h} \cdot \mathbf{y} \geq 0$. Let us introduce $\lambda=\min _{\mathbf{h} \in \mathbf{H}} \frac{\mathbf{h} \cdot \mathbf{y}}{\mathbf{h} \cdot \mathbf{x}}$ and observe that $\mathbf{c}=\mathbf{y}-\lambda \mathbf{x}_{s}$ satisfies 
$\mathbf{h} \cdot \mathbf{c} \geq 0$ for every $\mathbf{h} \in \mathbf{H}$. Hence $\mathbf{c} \in \mathbf{C}$. In particular $\mathbb{Q}_{>0} \mathbf{c}+\mathbb{Q}_{>0} \mathbf{X} \subseteq \mathbf{X}$. Let $\mathbf{K} \in \mathcal{K}^{\prime}$. Since $\mathbf{X} \subseteq \mathbf{K}$, we get $\mathbb{Q}>0 \mathbf{c}+\mathbb{Q}_{>0} \mathbf{x} \subseteq \mathbf{K}$. As $\mathcal{K}^{\prime}$ has the overlapping property we deduce that there exists $\mathbf{K}^{\prime} \in \mathcal{K}$ such that $\mathbf{K}^{\prime} \cap\left(\mathbb{Q}_{>0} \mathbf{c}\right)$ is non empty. Hence there exists $\mu \in \mathbb{Q}_{>0}$ such that $\mu \mathbf{c} \in \mathbf{K}^{\prime}$. Since $\mathbf{K}^{\prime}$ is a conic set we deduce that $\frac{1}{\mu}(\mu \mathbf{c}) \in \mathbf{K}^{\prime}$. Therefore $\mathbf{c} \in$ $\Sigma\left(\mathcal{K}^{\prime}\right)$. Moreover by definition of $\lambda$ we deduce that there exists $\mathbf{h} \in \mathbf{H}$ such that $\mathbf{c} \in \mathbf{V}_{\mathbf{h}}$. We deduce that $\mathbf{c} \in \Sigma\left(\mathbf{V}_{\mathbf{h}} \cap \mathcal{K}_{s}\right)$. Therefore, there exists $\mathbf{C} \in \mathcal{C}_{\mathbf{h}}$ such that $\mathbf{c} \in \mathbf{C}$. We have proved that $\mathbf{y} \in \Sigma(\mathcal{C})$. Therefore $\mathcal{C}$ is a complete extraction of $\mathcal{K}^{\prime}$.

The induction is proved.

We have proved Theorem E.2. 


\section{APPENDIX F \\ PRoOF OF THEOREM X.3}

In this section, we prove the following theorem.

Theorem X.3. Let $\mathbf{X}=\bigcup_{j=1}^{k}\left(\mathbf{b}_{j}+\mathbf{P}_{j}\right)$ where $\mathbf{b}_{j} \in \mathbb{Z}^{d}$ and $\mathbf{P}_{j} \subseteq \mathbb{Z}^{d}$ is a smooth periodic set. We assume that $\mathbf{X}$ is non empty and we introduce $r=\operatorname{dim}(\mathbf{X})$. If $\mathbf{X}$ is equivalent for $\equiv_{r}$ to a Presburger set then there exists a sequence $\left(\mathbf{Y}_{j}\right)_{1 \leq j \leq k}$ of linear sets $\mathbf{Y}_{j} \subseteq \mathbf{b}_{j}+\mathbf{P}_{j}$ such that $\mathbf{X} \equiv_{r} \bigcup_{j=1}^{k} \mathbf{p}_{j}+\mathbf{Y}_{j}$ for every sequence $\left(\mathbf{p}_{j}\right)_{1 \leq j \leq k}$ of vectors $\mathbf{p}_{j} \in \mathbf{P}_{j}$.

We first prove the following three lemmas.

Lemma F.1. For every periodic set $\mathbf{P} \subseteq \mathbb{Q}^{d}$ and for every vector $\mathbf{v} \in \mathbb{Q}^{d}$, we have $\mathbf{v} \in(\mathbf{P}-\mathbf{P}) \cap \lim (\mathbf{P})$ if, and only if there exists $\mathbf{p} \in \mathbf{P}$ such that $\mathbf{p}+\mathbb{N} \mathbf{v} \subseteq \mathbf{P}$.

Proof: Let $\mathbf{v} \in \mathbb{Q}^{d}$ and assume first that $\mathbf{p}+\mathbb{N} \mathbf{v} \subseteq \mathbf{P}$ for some $\mathbf{p} \in \mathbf{P}$. In this case $\mathbf{v} \in \lim (\mathbf{P})$ and from $\mathbf{v}=$ $(\mathbf{p}+\mathbf{v})-\mathbf{p}$ we deduce that $\mathbf{v} \in \mathbf{P}-\mathbf{P}$. Thus $\mathbf{v} \in(\mathbf{P}-\mathbf{P}) \cap$ $\lim (\mathbf{P})$. Conversely, let us consider $\mathbf{v} \in(\mathbf{P}-\mathbf{P}) \cap \lim (\mathbf{P})$. There exists $\mathbf{p}_{+}, \mathbf{p}_{-}$such that $\mathbf{v}=\mathbf{p}_{+}-\mathbf{p}_{-}$. Moreover there exists $\mathbf{q} \in \mathbf{P}$ and $n \in \mathbb{N}_{>0}$ such that $\mathbf{q}+n \mathbb{N} \mathbf{v} \subseteq \mathbf{P}$. Let us consider $\mathbf{p}=\mathbf{q}+n \mathbf{v}+(n-1) \mathbf{p}_{-}$and let us prove that $\mathbf{p}+\mathbb{N} \mathbf{v} \subseteq \mathbf{P}$. Let us consider $k \in \mathbb{N}$. The Euclidean divisor of $k$ by $n$ shows that there exists $q \in \mathbb{N}$ and $r \in\{0, \ldots, n-1\}$ such that $k=q n+r$. Note that $r \mathbf{p}_{-}+r \mathbf{v}=r \mathbf{p}_{+}$. Thus $(n-1) \mathbf{p}_{-}+r \mathbf{v}=r \mathbf{p}_{+}+(n-1-r) \mathbf{p}_{-} \in \mathbf{P}$. We deduce that $\mathbf{p}+k \mathbf{v}=(\mathbf{q}+n(q+1) \mathbf{v})+\left((n-1) \mathbf{p}_{-}+r \mathbf{v}\right) \in \mathbf{P}$. We have proved that $\mathbf{p}+\mathbb{N} \mathbf{v} \subseteq \mathbf{P}$. In particular $\mathbf{p} \in \mathbf{P}$.

Lemma F.2. Let $\mathbf{P}$ be a periodic set included in a Presburger set $\mathbf{S} \subseteq \mathbb{Z}^{d}$. We have:

$$
\operatorname{dim}((\mathbf{P}-\mathbf{P}) \cap \lim (\mathbf{P}) \backslash \mathbf{S})<\operatorname{dim}(\mathbf{S})
$$

Proof: Let $\mathbf{V}$ be the vector space generated by $\mathbf{P}$. Lemma X.2 shows that $\operatorname{dim}(\mathbf{P})=\operatorname{rank}(\mathbf{V})$. By replacing $\mathbf{S}$ by $\mathbf{S} \cap \mathbf{V}$ we can assume without loss of generality that $\mathbf{S} \subseteq \mathbf{V}$. Since the Presburger arithmetic admits a quantifier elimination algorithm, a quantifier free formula in disjunctive normal form shows that $\mathbf{S}$ can be decomposed into a finite union $\bigcup_{j=1}^{k}\left(\mathbf{R}_{j} \cap \mathbf{X}_{j}\right)$ where $\mathbf{R}_{j}$ is the set of vectors $\mathbf{z} \in \mathbb{Z}^{d} \cap \mathbf{V}$ satisfying a conjunction of formulas of the form $\mathbf{h} \cdot \mathbf{z} \in c+m \mathbb{Z}$ with $\mathbf{h} \in \mathbb{Z}^{d}, c \in \mathbb{Z}$ and $m \in \mathbb{N}_{>0}$, and where $\mathbf{X}_{j}$ is a subset of $\mathbf{V}$ such that there exists a finite set $A_{j} \subseteq \mathbb{Q}^{d} \times\{>, \geq\} \times \mathbb{Q}$ such that:

$$
\mathbf{X}_{j}=\left\{\mathbf{v} \in \mathbf{V} \mid \bigwedge_{(\mathbf{h}, \#, c) \in A_{j}} \mathbf{h} \cdot \mathbf{v} \# c\right\}
$$

We can assume that for every $(\mathbf{h}, \#, c) \in A_{j}$ there exists a vector $\mathbf{v} \in \mathbf{V}$ such that $\mathbf{h} \cdot \mathbf{v} \neq 0$ since otherwise the constraint $\mathbf{h} \cdot \mathbf{v} \# c$ reduces to $0 \# c$. Without loss of generality we can also assume that $\mathbf{R}_{j}$ is non empty. Let $\mathbf{r}_{j} \in \mathbf{R}_{j}$ and observe that $\mathbf{L}_{j}=\mathbf{R}_{j}-\mathbf{r}_{j}$ is a lattice that generates $\mathbf{V}$ since for every $\mathbf{v} \in \mathbf{V}$ there exists $m \in \mathbb{N}_{>0}$ such that $n \mathbf{v} \in \mathbf{L}_{j}$. We introduce the lattice $\mathbf{L}=\bigcap_{j=1}^{k} \mathbf{L}_{j}$. By considering a product of the natural numbers $m \in \mathbb{N}_{>0}$ (one for each $j$ ), we deduce that for every $\mathbf{v} \in \mathbf{V}$ there exists $m \in \mathbb{N}_{>0}$ such that $m \mathbf{v} \in \mathbf{L}$.

Let $\mathbf{v} \in(\mathbf{P}-\mathbf{P}) \cap \lim (\mathbf{P})$. Lemma F.1 shows that there exists $\mathbf{p} \in \mathbf{P}$ such that $\mathbf{p}+\mathbb{N} \mathbf{v} \subseteq \mathbf{P}$. By replacing $\mathbf{p}$ by a vector in $\mathbb{N}_{>0} \mathbf{p}$ we can assume that $\mathbf{p} \in \mathbf{L}$. Since $\mathbf{v} \in \mathbf{P}-\mathbf{P} \subseteq \mathbf{V}$, we deduce that there exists $m \in \mathbb{N}_{>0}$ such that $m \mathbf{v} \in \mathbf{L}$. Since $\mathbf{p}+\mathbf{v}+m \mathbb{N} \mathbf{v} \subseteq \mathbf{P} \subseteq \mathbf{S}$, there exists $j \in\{1, \ldots, k\}$ and an infinite subset $N \subseteq \mathbb{N}$ such that $\mathbf{p}+\mathbf{v}+m N \mathbf{v} \subseteq \mathbf{R}_{j} \cap \mathbf{X}_{j}$. Let $n \in N$ and observe that $\mathbf{p}+\mathbf{v}+m n \mathbf{v} \in \mathbf{r}_{j}+\mathbf{L}_{j}$ and from $\mathbf{p}, n m \mathbf{v} \in \mathbf{L} \subseteq \mathbf{L}_{j}$ we get $\mathbf{v} \in \mathbf{r}_{j}+\mathbf{L}_{j}=\mathbf{R}_{j}$. Moreover, since $N$ is infinite and $\mathbf{p}+\mathbf{v}+m N \mathbf{v} \subseteq \mathbf{X}_{j}$ we deduce that $\mathbf{v}$ is in the following set $\tilde{\mathbf{X}}_{j}$ :

$$
\tilde{\mathbf{X}}_{j}=\left\{\mathbf{v} \in \mathbf{V} \mid \bigwedge_{(\mathbf{h}, \#, c) \in A_{j}} \mathbf{h} \cdot \mathbf{v} \geq 0\right\}
$$

We have proved that $(\mathbf{P}-\mathbf{P}) \cap \lim (\mathbf{P}) \subseteq \tilde{\mathbf{S}}$ where $\tilde{\mathbf{S}}=$ $\bigcup_{j=1}^{k}\left(\mathbf{R}_{j} \cap \tilde{\mathbf{X}}_{j}\right)$. Thus $\operatorname{dim}((\mathbf{P}-\mathbf{P}) \cap \lim (\mathbf{P}) \backslash \mathbf{S}) \leq \operatorname{dim}(\tilde{\mathbf{S}} \backslash \mathbf{S})$. From Lemma D.1 since $\equiv_{r}$ is distributive over $\cup$ and $\cap$, we get $\mathbf{S} \equiv_{r} \tilde{\mathbf{S}}$. Thus $\operatorname{dim}((\mathbf{P}-\mathbf{P}) \cap \lim (\mathbf{P}) \backslash \mathbf{S})<r$.

Lemma F.3. Let $\left(\mathbf{m}_{r}\right)_{1 \leq r \leq n}$ be a sequence of vectors $\mathbf{m}_{r} \in$ $\mathbb{Z}^{d}$ and let $\mathbf{M}_{r}=\mathbb{N m}_{1}+\cdots+\mathbb{N m}_{r}$ for every $r \in\{1, \ldots, n\}$. If $\mathbf{M}_{n}$ is included in $\bigcup_{j=1}^{k} \mathbf{b}_{j}+\mathbf{P}_{j}$ where $\mathbf{b}_{j} \in \mathbb{Z}^{d}$ and $\mathbf{P}_{j} \subseteq$ $\mathbb{Z}^{d}$ is a well-limit periodic set then there exists $j \in\{1, \ldots, k\}$ such that $\mathbf{M}_{n} \cap\left(\mathbf{b}_{j}+\mathbf{P}_{j}\right)$ is non empty and such that $\left(\mathbf{m}_{r}+\right.$ $\left.\mathbf{M}_{r}\right) \cap \lim \left(\mathbf{P}_{j}\right)$ is non empty for every $r \in\{1, \ldots, n\}$.

Proof: We prove the lemma by induction over $n \in \mathbb{N}$. The rank $n=0$ is immediate. Assume the rank $n \in \mathbb{N}$ proved and let us consider a sequence $\left(\mathbf{m}_{r}\right)_{1 \leq r \leq n+1}$ of vectors $\mathbf{m}_{r} \in \mathbb{Z}^{d}$ and let $\mathbf{M}_{r}=\mathbb{N m}_{1}+\cdots+\mathbb{N m}_{r}$ for every $r \in\{1, \ldots, n+1\}$. Assume $\mathbf{M}_{n+1}$ is included in $\bigcup_{j=1}^{k} \mathbf{b}_{j}+\mathbf{P}_{j}$ where $\mathbf{b}_{j} \in \mathbb{Z}^{d}$ and $\mathbf{P}_{j} \subseteq \mathbb{Z}^{d}$ is a well-limit periodic set. Let $t \in \mathbb{N}$ and observe that $\mathbf{M}_{n} \subseteq \bigcup_{j=1}^{k} \mathbf{b}_{j}-t \mathbf{m}_{n+1}+\mathbf{P}_{j}$. By induction there exists $j \in\{1, \ldots, k\}$ such that $\mathbf{M}_{n} \cap\left(\mathbf{b}_{j}-t \mathbf{m}_{n+1}+\right.$ $\left.\mathbf{P}_{j}\right)$ is non empty and such that $\left(\mathbf{m}_{r}+\mathbf{M}_{r}\right) \cap \lim \left(\mathbf{P}_{j}\right)$ is non empty for every $r \in\{1, \ldots, n\}$. We deduce that there exists $j \in\{1, \ldots, k\}$ and an infinite subset $T \subseteq \mathbb{N}$ such that $\left(\mathbf{m}_{r}+\mathbf{M}_{r}\right) \cap \lim \left(\mathbf{P}_{j}\right)$ is non empty for every $r \in\{1, \ldots, n\}$ and such that $\mathbf{M}_{n} \cap\left(\mathbf{b}_{j}-t \mathbf{m}_{n+1}+\mathbf{P}_{j}\right)$ is non empty for every $t \in T$. Since $\mathbf{M}_{n}+t \mathbf{m}_{n+1} \subseteq \mathbf{M}_{n+1}$ we deduce that $\mathbf{M}_{n+1} \cap\left(\mathbf{b}_{j}+\mathbf{P}_{j}\right)$ is non empty. For every $t \in T$ there exists $\mathbf{k}_{t} \in \mathbf{M}_{n}$ such that $\mathbf{v}_{t}=\mathbf{k}_{t}-\mathbf{b}_{j}+t \mathbf{m}_{n+1}$ in in $\mathbf{P}_{j}$. As $\mathbf{M}_{n}$ is finitely generated and $\mathbf{P}_{j}$ is a well-limit periodic set, we deduce that there exists $t<t^{\prime}$ such that $\mathbf{k}_{t^{\prime}}-\mathbf{k}_{t} \in \mathbf{M}_{n}$ and $\mathbf{v}_{t^{\prime}}-\mathbf{v}_{t} \in \lim \left(\mathbf{P}_{j}\right)$. Observe that this last vector is equal to $\mathbf{k}_{t^{\prime}}-\mathbf{k}_{t}+\left(t^{\prime}-t\right) \mathbf{m}_{n+1}$ which is in $\mathbf{m}_{n+1}+\mathbf{M}_{n+1}$. So we have proved the rank $n+1$. Therefore, the lemma is proved by induction.

Now, let us prove Theorem X.3. We consider a non-empty set $\mathbf{X}=\bigcup_{j=1}^{k} \mathbf{b}_{j}+\mathbf{P}_{j}$ where $\mathbf{b}_{j} \in \mathbb{Z}^{d}$ and $\mathbf{P}_{j} \subseteq \mathbb{Z}^{d}$ is a smooth periodic set. We introduce the definable conic set $\mathbf{K}_{j}=\lim \left(\mathbf{P}_{j}\right)$. We denote by $r$ the dimension of $\mathbf{X}$. Note that $k>0$ and $r \in\{1, \ldots, d\}$ since $\mathbf{X}$ is non empty. We introduce the lattices $\mathbf{L}_{j}=\mathbf{P}_{j}-\mathbf{P}_{j}$. We denote by $\mathbf{V}_{j}$ the vector space 
generated by $\mathbf{P}_{j}$. We introduce the set $J=\{j \in\{1, \ldots, k\} \mid$ $\left.\operatorname{rank}\left(\mathbf{V}_{j}\right)=r\right\}$, the class $\mathcal{V}=\left\{\mathbf{V}_{j} \mid j \in J\right\}$. For every vector space $\mathbf{V} \in \mathcal{V}$ we introduce the set $J_{\mathbf{V}}=\left\{j \in J \mid \mathbf{V}=\mathbf{V}_{j}\right\}$, the lattice $\mathbf{L}_{\mathbf{V}}=\bigcap_{j \in J_{\mathbf{V}}} \mathbf{L}_{j}$. For every $\mathbf{V} \in \mathcal{V}$ and for every $\mathbf{z} \in \mathbb{Z}^{d}$ we introduce the set $J_{\mathbf{V}, \mathbf{z}}=\left\{j \in J_{\mathbf{V}} \mid \mathbf{z} \in \mathbf{b}_{j}+\mathbf{L}_{j}\right\}$ and the finite class $\mathcal{K}_{\mathbf{V}, \mathbf{z}}=\left\{\mathbf{K}_{j} \mid j \in J_{\mathbf{V}, \mathbf{z}}\right\}$.

Lemma F.4. For every $\mathbf{V} \in \mathcal{V}$ and for every $\mathbf{z} \in \mathbb{Z}^{d}$, we have:

$$
\mathbf{L}_{\mathbf{V}} \cap(\mathbf{X}-\mathbf{z}) \equiv_{r} \mathbf{L}_{\mathbf{V}} \cap \bigcup_{j \in J_{\mathbf{V}, \mathbf{z}}} \mathbf{b}_{j}-\mathbf{z}+\mathbf{P}_{j}
$$

Proof: Let us consider $j \in\{1, \ldots, k\}$ such that the dimension of the intersection $\mathbf{L}_{\mathbf{V}} \cap\left(\mathbf{b}_{j}-\mathbf{z}+\mathbf{P}_{j}\right)$ is greater or equal to $r$ and let us prove that $j \in J_{\mathbf{V}, \mathbf{z}}$. In that case, this intersection is non empty and thus it contains a vector $\mathbf{x}$. We deduce that the intersection is included in $\mathbf{x}+\left(\mathbf{V} \cap \mathbf{V}_{j}\right)$. Hence $\operatorname{rank}\left(\mathbf{V} \cap \mathbf{V}_{j}\right) \geq r$. From $\mathbf{V} \cap \mathbf{V}_{j} \subseteq \mathbf{V}$ and $\operatorname{rank}(\mathbf{V})=r$ we get $\mathbf{V} \cap \mathbf{V}_{j}=\mathbf{V}$. Hence $\mathbf{V} \subseteq \mathbf{V}_{j}$. As $\operatorname{rank}(\mathbf{V})=r$ and $\operatorname{rank}\left(\mathbf{V}_{j}\right) \leq r$ we deduce that $\mathbf{V}=\mathbf{V}_{j}$. Thus $j \in J_{\mathbf{V}}$. Moreover, since $\mathbf{x} \in \mathbf{L}_{\mathbf{V}} \cap\left(\mathbf{b}_{j}-\mathbf{z}+\mathbf{P}_{j}\right)$ we deduce that $\mathbf{b}_{j}-\mathbf{z} \in \mathbf{x}+\mathbf{L}_{j} \subseteq \mathbf{L}_{j}$ as $\mathbf{x} \in \mathbf{L}_{\mathbf{V}} \subseteq \mathbf{L}_{j}$. Thus $j \in J_{\mathbf{V}, \mathbf{z}}$. We deduce the relations:

$$
\begin{aligned}
\mathbf{L}_{\mathbf{V}} \cap(\mathbf{X}-\mathbf{z}) & =\mathbf{L}_{\mathbf{V}} \cap \bigcup_{j=1}^{k}\left(\mathbf{b}_{j}-\mathbf{z}+\mathbf{P}_{j}\right) \\
& =\bigcup_{j=1}^{k} \mathbf{L}_{\mathbf{V}} \cap\left(\mathbf{b}_{j}-\mathbf{z}+\mathbf{P}_{j}\right) \\
& \equiv_{r} \bigcup_{j \in J_{\mathbf{V}, \mathbf{z}}} \mathbf{L}_{\mathbf{V}} \cap\left(\mathbf{b}_{j}-\mathbf{z}+\mathbf{P}_{j}\right) \\
& =\mathbf{L} \mathbf{V} \cap \bigcup_{j \in J \mathbf{V}, \mathbf{z}}\left(\mathbf{b}_{j}-\mathbf{z}+\mathbf{P}_{j}\right)
\end{aligned}
$$

We have proved the lemma.

Lemma F.5. If there exists a Presburger set $\mathbf{S} \subseteq \mathbb{Z}^{d}$ such that $\mathbf{X} \equiv_{r} \mathbf{S}$ then for every $\mathbf{V} \in \mathcal{V}$ and for every $\mathbf{z} \in \mathbb{Z}^{d}$ we have:

$$
\mathbf{L}_{\mathbf{V}} \cap \Sigma\left(\mathcal{K}_{\mathbf{V}, \mathbf{z}}\right) \equiv_{r} \mathbf{L}_{\mathbf{V}} \cap \bigcup_{j \in J_{\mathbf{V}, \mathbf{z}}} \mathbf{b}_{j}-\mathbf{z}+\mathbf{P}_{j}
$$

Proof: Assume that there exists a Presburger set $\mathbf{S} \subseteq \mathbb{Z}^{d}$ such that $\mathbf{X} \equiv_{r} \mathbf{S}$. Lemma F.4 shows the following relation:

$$
\mathbf{L}_{\mathbf{V}} \cap(\mathbf{S}-\mathbf{z}) \equiv_{r} \mathbf{L} \mathbf{V} \cap \bigcup_{j \in J_{\mathbf{V}, \mathbf{z}}} \mathbf{b}_{j}-\mathbf{z}+\mathbf{P}_{j}
$$

Hence, there exists a Presburger set $\mathbf{D} \subseteq \mathbb{Z}^{d}$ such that $\operatorname{dim}(\mathbf{D})<r$ and such that the Presburger set $\mathbf{R}=\mathbf{L}_{\mathbf{V}} \cap(\mathbf{S}-\mathbf{z})$ satisfies $\mathbf{R} \backslash \mathbf{D} \subseteq \mathbf{L}_{\mathbf{V}} \cap \bigcup_{j \in J_{\mathbf{V}, \mathbf{z}}} \mathbf{b}_{j}-\mathbf{z}+\mathbf{P}_{j} \subseteq \mathbf{D} \cup \mathbf{R}$. Lemma F.2 shows that for every $j \in J_{\mathbf{V}, \mathbf{z}}$ there exists a Presburger set $\mathbf{D}_{j} \subseteq \mathbb{Z}^{d}$ such that $\operatorname{dim}\left(\mathbf{D}_{j}\right)<\operatorname{dim}\left(\mathbf{P}_{j}\right)$ and such that $\mathbf{b}_{j}-\mathbf{z}+\left(\mathbf{L}_{j} \cap \mathbf{K}_{j}\right) \subseteq \mathbf{D}_{j} \cup \mathbf{D} \cup \mathbf{R}$. Therefore $\mathbf{L}_{\mathbf{V}} \cap \bigcup_{j \in J_{\mathbf{V}, \mathbf{z}}} \mathbf{b}_{j}-\mathbf{z}+\left(\mathbf{L}_{j} \cap \mathbf{K}_{j}\right)$ is included in the union of $\mathbf{Z}=\mathbf{D} \cup \bigcup_{j \in J_{\mathbf{V}, \mathbf{z}}} \mathbf{D}_{j}$ and $\mathbf{R}$. We get the following inclusions:

$$
\mathbf{R} \backslash \mathbf{Z} \subseteq \mathbf{L}_{\mathbf{V}} \cap \bigcup_{j \in J_{\mathbf{V}, \mathbf{z}}} \mathbf{b}_{j}-\mathbf{z}+\left(\mathbf{L}_{j} \cap \mathbf{K}_{j}\right) \subseteq \mathbf{R} \cup \mathbf{Z}
$$

Since $\operatorname{dim}(\mathbf{Z})<r$ we deduce the following relation:

$$
\mathbf{L}_{\mathbf{V}} \cap \bigcup_{j \in J \mathbf{V}, \mathbf{z}} \mathbf{b}_{j}-\mathbf{z}+\left(\mathbf{L}_{j} \cap \mathbf{K}_{j}\right) \equiv_{r} \mathbf{L}_{\mathbf{V}} \cap \bigcup_{j \in J_{\mathbf{V}, \mathbf{z}}} \mathbf{b}_{j}-\mathbf{z}+\mathbf{P}_{j}
$$

Finally observe that for every $j \in J_{\mathbf{V}, \mathbf{z}}$, since $\mathbf{b}_{j}-\mathbf{z} \in \mathbf{L}_{j}$, we have $\mathbf{b}_{j}-\mathbf{z}+\left(\mathbf{L}_{j} \cap \mathbf{K}_{j}\right)=\mathbf{L}_{j} \cap\left(\mathbf{b}_{j}-\mathbf{z}+\mathbf{K}_{j}\right)$. Corollary D.2 shows that $\mathbf{L}_{j} \cap\left(\mathbf{b}_{j}-\mathbf{z}+\mathbf{K}_{j}\right) \equiv_{r} \mathbf{L}_{j} \cap \mathbf{K}_{j}$.

Lemma F.6. Let $\mathbf{V} \in \mathcal{V}$ and $\mathbf{z} \in \mathbb{Z}^{d}$ such that:

$$
\mathbf{L}_{\mathbf{V}} \cap \Sigma\left(\mathcal{K}_{\mathbf{V}, \mathbf{z}}\right) \equiv_{r} \mathbf{L}_{\mathbf{V}} \cap \bigcup_{j \in J_{\mathbf{V}, \mathbf{z}}} \mathbf{b}_{j}-\mathbf{z}+\mathbf{P}_{j}
$$

Then the class $\mathcal{K}_{\mathbf{V}, \mathbf{z}}$ has the overlapping property.

Proof: There exists a Presburger set $\mathbf{D} \subseteq \mathbb{Z}^{d}$ such that $\operatorname{dim}(\mathbf{D})<r$ and such that the Presburger set $\mathbf{S}=\mathbf{L}_{\mathbf{V}} \cap$ $\Sigma\left(\mathcal{K}_{\mathbf{V}, \mathbf{z}}\right)$ and the set $\mathbf{R}=\mathbf{L}_{\mathbf{V}} \cap \bigcup_{j \in J_{\mathbf{V}, \mathbf{z}}} \mathbf{b}_{j}-\mathbf{z}+\mathbf{P}_{j}$ satisfy $\mathbf{R} \backslash \mathbf{D} \subseteq \mathbf{S} \subseteq \mathbf{R} \cup \mathbf{D}$.

Let us consider $j_{0} \in J_{\mathbf{V}, \mathbf{z}}$ and a sequence $\mathbf{v}_{1}, \ldots, \mathbf{v}_{n}$ of vectors $\mathbf{v}_{n} \in \mathbb{Q}^{d}$ such that $\mathbb{Q}_{>0} \mathbf{v}_{1}+\cdots+\mathbb{Q}_{>0} \mathbf{v}_{n} \subseteq \mathbf{K}_{j_{0}}$ and let us prove that there exists $\mathbf{K} \in \mathcal{K}_{\mathbf{V}, \mathbf{z}}$ such that $\mathbf{K} \cap\left(\mathbb{Q}_{>0} \mathbf{v}_{1}+\cdots+\mathbb{Q}_{>0} \mathbf{v}_{r}\right) \neq \emptyset$ for every $r \in\{1, \ldots, n\}$. By extending the sequence we can assume that $\mathbf{v}_{1}, \ldots, \mathbf{v}_{n}$ generates $\mathbf{V}$. Moreover, by replacing vectors $\mathbf{v}_{r}$ by vectors in $\mathbb{N}_{>0} \mathbf{v}_{r}$ we can assume without loss of generality that $\mathbf{v}_{r} \in \mathbf{L}_{\mathbf{V}}$. Therefore $\mathbf{v}=\mathbf{v}_{1}+\cdots+\mathbf{v}_{n}$ satisfies $\mathbf{v}+\mathbb{N}_{\mathbf{v}_{1}}+\cdots+\mathbb{N}_{n} \subseteq \mathbf{L}_{\mathbf{V}} \cap \mathbf{K}_{j_{0}} \subseteq \mathbf{S} \subseteq \mathbf{R} \cup \mathbf{D}$. By decomposing $\mathbf{D}$ into linear sets, since $\mathbf{v}_{1}, \ldots, \mathbf{v}_{n}$ generates $\mathbf{V}$, Lemma F.3 shows that there exists $j \in J_{\mathbf{V}, \mathbf{z}}$ such that $\left(\mathbf{v}+\mathbb{N}_{1}+\cdots+\mathbb{N}_{n}\right) \cap\left(\mathbf{b}_{j}-\mathbf{z}+\mathbf{P}_{j}\right) \neq \emptyset$ and such that $\left(\mathbb{N}_{>0} \mathbf{v}_{1}+\cdots+\mathbb{N}_{>0} \mathbf{v}_{r}\right) \cap \mathbf{K}_{j} \neq \emptyset$ for every $r \in\{1, \ldots, n\}$. We have proved that $\mathcal{K}_{\mathbf{V}, \mathbf{z}}$ has the overlapping property.

Lemma F.7. For every $\mathbf{V} \in \mathcal{V}$ and for every $\mathbf{z} \in \mathbb{Z}^{d}$ we have:

$$
\mathbf{L}_{\mathbf{V}} \cap \bigcup_{j \in J_{\mathbf{V}, \mathbf{z}}} \mathbf{b}_{j}-\mathbf{z}+\left(\mathbf{L}_{j} \cap \mathbf{K}_{j}\right) \equiv_{r} \mathbf{L}_{\mathbf{V}} \cap \Sigma\left(\mathcal{K}_{\mathbf{V}, \mathbf{z}}\right)
$$

Proof: We observe that for every $j \in J_{\mathbf{V}}$, the intersection $\mathbf{L}_{\mathbf{V}} \cap\left(\mathbf{b}_{j}-\mathbf{z}+\mathbf{L}_{j}\right)$ is equal to $\mathbf{L}_{\mathbf{V}}$. We deduce the following equality:

$$
\begin{aligned}
& \mathbf{L}_{\mathbf{V}} \cap \bigcup_{j \in J_{\mathbf{V}, \mathbf{z}}} \mathbf{b}_{j}-\mathbf{z}+\left(\mathbf{L}_{j} \cap \mathbf{K}_{j}\right) \\
& =\bigcup_{j \in J_{\mathbf{V}, \mathbf{z}}} \mathbf{L}_{\mathbf{V}} \cap\left(\mathbf{b}_{j}-\mathbf{z}+\left(\mathbf{L}_{j} \cap \mathbf{K}_{j}\right)\right)
\end{aligned}
$$

Since $\mathbf{b}_{j}-\mathbf{z} \in \mathbf{L}_{j}$ we get $\mathbf{b}_{j}-\mathbf{z}+\left(\mathbf{L}_{j} \cap \mathbf{K}_{j}\right)=\mathbf{L}_{j} \cap\left(\mathbf{b}_{j}-\mathbf{z}+\right.$ $\left.\mathbf{K}_{j}\right)$. Corollary D. 1 shows that $\mathbf{L}_{j} \cap\left(\mathbf{b}_{j}-\mathbf{z}+\mathbf{K}_{j}\right) \equiv_{r} \mathbf{L}_{j} \cap \mathbf{K}_{j}$. Hence $\mathbf{L}_{\mathbf{V}} \cap\left(\mathbf{b}_{j}-\mathbf{z}+\left(\mathbf{L}_{j} \cap \mathbf{K}_{j}\right)\right) \equiv_{r} \mathbf{L}_{\mathbf{V}} \cap \mathbf{K}_{j}$. We have proved:

$$
\begin{aligned}
& \bigcup_{j \in J_{\mathbf{V}, \mathbf{z}}} \mathbf{L}_{\mathbf{V}} \cap\left(\mathbf{b}_{j}-\mathbf{z}+\left(\mathbf{L}_{j} \cap \mathbf{K}_{j}\right)\right) \\
& \equiv_{r} \bigcup_{j \in J_{\mathbf{V}, \mathbf{z}}} \mathbf{L}_{\mathbf{V}} \cap \mathbf{K}_{j}=\mathbf{L}_{\mathbf{V}} \cap \Sigma\left(\mathcal{K}_{\mathbf{V}, \mathbf{z}}\right)
\end{aligned}
$$

We deduce the lemma. 
Lemma F.8. If $\mathcal{K}_{\mathbf{V}, \mathbf{z}}$ has a complete extraction for every $\mathbf{V} \in$ $\mathcal{V}$ and for every $\mathbf{z} \in \mathbb{Z}^{d}$ then there exists a finite sequence $\left(\mathbf{C}_{j}\right)_{1 \leq j \leq k}$ of finitely generated conic sets $\mathbf{C}_{j} \subseteq \mathbf{K}_{j}$ such that $\bigcup_{j=1}^{k} \mathbf{b}_{j}+\mathbf{L}_{j} \cap \mathbf{K}_{j} \equiv \bigcup_{j=1}^{k} \mathbf{b}_{j}+\mathbf{L}_{j} \cap \mathbf{C}_{j}$.

Proof: Let $\mathbf{V} \in \mathcal{V}$ and $\mathbf{z} \in \mathbb{Z}^{d}$. From Lemma F.7 we deduce the following relation:

$$
\mathbf{L}_{\mathbf{V}} \cap \bigcup_{j \in J_{\mathbf{V}, \mathbf{z}}} \mathbf{b}_{j}-\mathbf{z}+\left(\mathbf{L}_{j} \cap \mathbf{K}_{j}\right) \equiv_{r} \mathbf{L}_{\mathbf{V}} \cap \Sigma\left(\mathcal{K}_{\mathbf{V}, \mathbf{z}}\right)
$$

Since $\mathcal{K}_{\mathbf{V}, \mathbf{z}}$ has a complete extraction, there exists a sequence $\left(\mathbf{C}_{\mathbf{V}, \mathbf{z}, j}\right)_{j \in J_{\mathbf{V}, \mathbf{z}}}$ of finitely generated conic sets $\mathbf{C}_{\mathbf{V}, \mathbf{z}, j} \subseteq \mathbf{K}_{j}$ such that $\bigcup_{j \in J_{\mathbf{V}, \mathbf{z}}} \mathbf{C}_{\mathbf{V}, \mathbf{z}, j}=\Sigma\left(\mathcal{K}_{\mathbf{V}, \mathbf{z}}\right)$. Since for every $j \in J_{\mathbf{V}, \mathbf{z}}$ we have $\mathbf{L}_{\mathbf{V}} \cap\left(\mathbf{b}_{j}-\mathbf{z}+\mathbf{L}_{j}\right)=\mathbf{L}_{\mathbf{V}}$, we deduce:

$$
\begin{aligned}
\mathbf{L}_{\mathbf{V}} \cap \Sigma\left(\mathcal{K}_{\mathbf{V}, \mathbf{z}}\right) & =\bigcup_{j \in J_{\mathbf{V}, \mathbf{z}}} \mathbf{L}_{\mathbf{V}} \cap \mathbf{C}_{\mathbf{V}, \mathbf{z}, j} \\
& =\mathbf{L}_{\mathbf{V}} \cap\left(\bigcup_{j \in J_{\mathbf{V}, \mathbf{z}}}\left(\mathbf{b}_{j}-\mathbf{z}+\mathbf{L}_{j}\right) \cap \mathbf{C}_{\mathbf{V}, \mathbf{z}, j}\right)
\end{aligned}
$$

Lemma D.1 shows that $\left(\mathbf{b}_{j}-\mathbf{z}+\mathbf{L}_{j}\right) \cap \mathbf{C}_{\mathbf{V}, \mathbf{z}, j} \equiv_{r} \mathbf{b}_{j}-\mathbf{z}+$ $\left(\mathbf{L}_{j} \cap \mathbf{C}_{\mathbf{V}, \mathbf{z}, j}\right)$ for every $j \in J_{\mathbf{V}, \mathbf{z}}$. We have proved:

$$
\mathbf{L}_{\mathbf{V}} \cap \Sigma\left(\mathcal{K}_{\mathbf{V}, \mathbf{z}}\right) \equiv_{r} \mathbf{L}_{\mathbf{V}} \cap \bigcup_{j \in J_{\mathbf{V}, \mathbf{z}}} \mathbf{b}_{j}-\mathbf{z}+\left(\mathbf{L}_{j} \cap \mathbf{C}_{\mathbf{V}, \mathbf{z}, j}\right)
$$

Let us introduce a finite set $\mathbf{Z}_{\mathbf{V}} \subseteq \mathbb{Z}^{d}$ such that $\sum_{j \in J_{\mathbf{V}}} \mathbf{b}_{j}+$ $\mathbf{L}_{j}=\mathbf{Z}_{\mathbf{V}}+\mathbf{L}_{\mathbf{V}}$. We consider the sequence $\left(\mathbf{C}_{j}\right)_{1 \leq j \leq k}$ of finitely generated conic sets defined by $\mathbf{C}_{j}=\{\mathbf{0}\}$ if $j \notin J$ and defined for every $j \in J$ by:

$$
\mathbf{C}_{j}=\sum_{\mathbf{z} \in \mathbf{Z}_{\mathbf{V}_{j}}} \mathbf{C}_{\mathbf{V}_{j}, \mathbf{z}, j}
$$

Observe that $\mathbf{C}_{j} \subseteq \mathbf{K}_{j}$ for every $j \in\{1, \ldots, k\}$. In particular $\mathbf{L}_{j} \cap \mathbf{C}_{j} \subseteq \mathbf{L}_{j} \cap \mathbf{K}_{j}$. We consider the sequence $\left(\mathbf{M}_{j}\right)_{1 \leq j \leq k}$ of sets $\mathbf{M}_{j}=\mathbf{L}_{j} \cap \mathbf{C}_{j}$. Since $\mathbf{L}_{\mathbf{V}} \cap\left(\mathbf{b}_{j}-\mathbf{z}+\mathbf{M}_{j}\right)$ is empty for every $j \in J_{\mathbf{V}} \backslash J_{\mathbf{V}, \mathbf{z}}$ we deduce:

$$
\begin{aligned}
& \mathbf{L}_{\mathbf{V}} \cap\left(\bigcup_{j \in J_{\mathbf{V}}} \mathbf{b}_{j}-\mathbf{z}+\left(\mathbf{L}_{j} \cap \mathbf{K}_{j}\right)\right) \\
& \equiv_{r} \mathbf{L}_{\mathbf{V}} \cap \bigcup_{j \in J_{\mathbf{V}, \mathbf{z}}} \mathbf{b}_{j}-\mathbf{z}+\mathbf{M}_{j} \\
& =\mathbf{L}_{\mathbf{V}} \cap \bigcup_{j \in J_{\mathbf{V}}} \mathbf{b}_{j}-\mathbf{z}+\mathbf{M}_{j}
\end{aligned}
$$

Since $\sum_{j \in J_{\mathbf{V}}} \mathbf{b}_{j}+\mathbf{L}_{j}=\mathbf{Z}_{\mathbf{V}}+\mathbf{L}_{\mathbf{V}}$, we deduce the relation $\bigcup_{j \in J_{\mathbf{V}}} \mathbf{b}_{j}+\left(\mathbf{L}_{j} \cap \mathbf{K}_{j}\right) \equiv_{r} \bigcup_{j \in J_{\mathbf{V}}} \mathbf{b}_{j}+\mathbf{M}_{j}$. Therefore $\bigcup_{j \in J} \mathbf{b}_{j}+\left(\mathbf{L}_{j} \cap \mathbf{K}_{j}\right) \equiv_{r} \bigcup_{j \in J} \mathbf{b}_{j}+\mathbf{M}_{j}$. Since $\operatorname{dim}\left(\mathbf{b}_{j}+\left(\mathbf{L}_{j} \cap\right.\right.$ $\left.\left.\mathbf{K}_{j}\right)\right)<r$ and $\operatorname{dim}\left(\mathbf{b}_{j}+\mathbf{M}_{j}\right)<r$ for every $j \in\{1, \ldots, k\} \backslash J$ we deduce that $\mathbf{b}_{j}+\left(\mathbf{L}_{j} \cap \mathbf{K}_{j}\right) \equiv_{r} \emptyset$ and $\mathbf{b}_{j}+\mathbf{M}_{j} \equiv_{r} \emptyset$. We have proved the following relation:

$$
\bigcup_{j=1}^{k} \mathbf{b}_{j}+\left(\mathbf{L}_{j} \cap \mathbf{K}_{j}\right) \equiv_{r} \bigcup_{j=1}^{k} \mathbf{b}_{j}+\mathbf{M}_{j}
$$

In the previous relation, the relation $\equiv_{r}$ can be replaced by $\equiv$ since the set $\mathbf{Y}=\bigcup_{j=1}^{k} \mathbf{b}_{j}+\left(\mathbf{L}_{j} \cap \mathbf{K}_{j}\right)$ satisfies $\operatorname{dim}(\mathbf{Y})=r$.
Now let us prove Theorem X.3. Assume that $\mathbf{X}$ is equivalent for $\equiv_{r}$ to a Presburger set. Lemmas F.5 and F.6 show that $\mathcal{K}_{\mathbf{V}, \mathbf{z}}$ has the overlapping property for every $\mathbf{V} \in \mathcal{V}$ and $\mathbf{z} \in \mathbb{Z}^{d}$. Theorem E.2 shows that $\mathcal{K}_{\mathbf{V}, \mathbf{z}}$ has the complete extraction property. Lemma F.8 shows that there exists a finite sequence $\left(\mathbf{C}_{j}\right)_{1 \leq j \leq k}$ of finitely generated conic sets $\mathbf{C}_{j} \subseteq \mathbf{K}_{j}$ such that $\mathbf{X} \equiv{ }_{r} \bigcup_{j=1}^{\bar{k}} \mathbf{b}_{j}+\left(\mathbf{L}_{j} \cap \mathbf{C}_{j}\right)$.

Let us introduce the periodic set $\mathbf{Q}_{j}=\mathbf{L}_{j} \cap \mathbf{C}_{j}$. Since the conic set generated by $\mathbf{Q}_{j}$ is $\mathbf{C}_{j}$ which is finitely generated, Lemma V.5 shows that $\mathbf{Q}_{j}$ is finitely generated. Lemma F.1 shows that for every $\mathbf{v} \in \mathbf{Q}_{j}$ there exists $\mathbf{p} \in \mathbf{P}_{j}$ such that $\mathbf{p}+\mathbb{N} \mathbf{v} \subseteq \mathbf{P}_{j}$. Since $\mathbf{Q}_{j}$ is finitely generated, there exists $\mathbf{y}_{j} \in \mathbf{b}_{j}+\mathbf{P}_{j}$ such that the linear set $\mathbf{Y}_{j}=\mathbf{y}_{j}+\mathbf{Q}_{j}$ is included in $\mathbf{b}_{j}+\mathbf{P}_{j}$.

Now, let us consider a sequence $\left(\mathbf{p}_{j}\right)_{1 \leq j \leq k}$ of vectors $\mathbf{p}_{j} \in$ $\mathbf{P}_{j}$. Note that $\mathbf{p}_{j}+\mathbf{Y}_{j}=\mathbf{y}_{j}+\left(\mathbf{L}_{j} \cap\left(\mathbf{p}_{j}+\mathbf{C}_{j}\right)\right)$. Note that the vector space $\mathbf{W}_{j}$ generated by $\mathbf{C}_{j}$ is included in $\mathbf{V}_{j}$. If the inclusion is strict then $\operatorname{rank}\left(\mathbf{W}_{j}\right)<r$ and we get $\mathbf{p}_{j}+\mathbf{Y}_{j} \equiv_{r}$ $\emptyset \equiv_{r} \mathbf{b}_{j}+\mathbf{Q}_{j}$. Otherwise, if $\mathbf{W}_{j}=\mathbf{V}_{j}$ then Corollary D.2 shows that $\mathbf{p}_{j}+\left(\mathbf{y}_{j}-\mathbf{b}_{j}\right)+\mathbf{C}_{j} \equiv_{r} \mathbf{C}_{j}$. Thus $\mathbf{p}_{j}+\mathbf{Y}_{j} \equiv_{r}$ $\mathbf{b}_{j}+\mathbf{Q}_{j}$. We have proved that $\mathbf{X} \equiv_{r} \bigcup_{j=1}^{k} \mathbf{p}_{j}+\mathbf{Y}_{j}$. 